Materials Science and Engineering Laboratory

NAS-NRC

Assessment Panel

April 6-7, 1995

NISTIR 5581

U.S. Department of Commerce Technology Administration National Institute of Standards and Technology
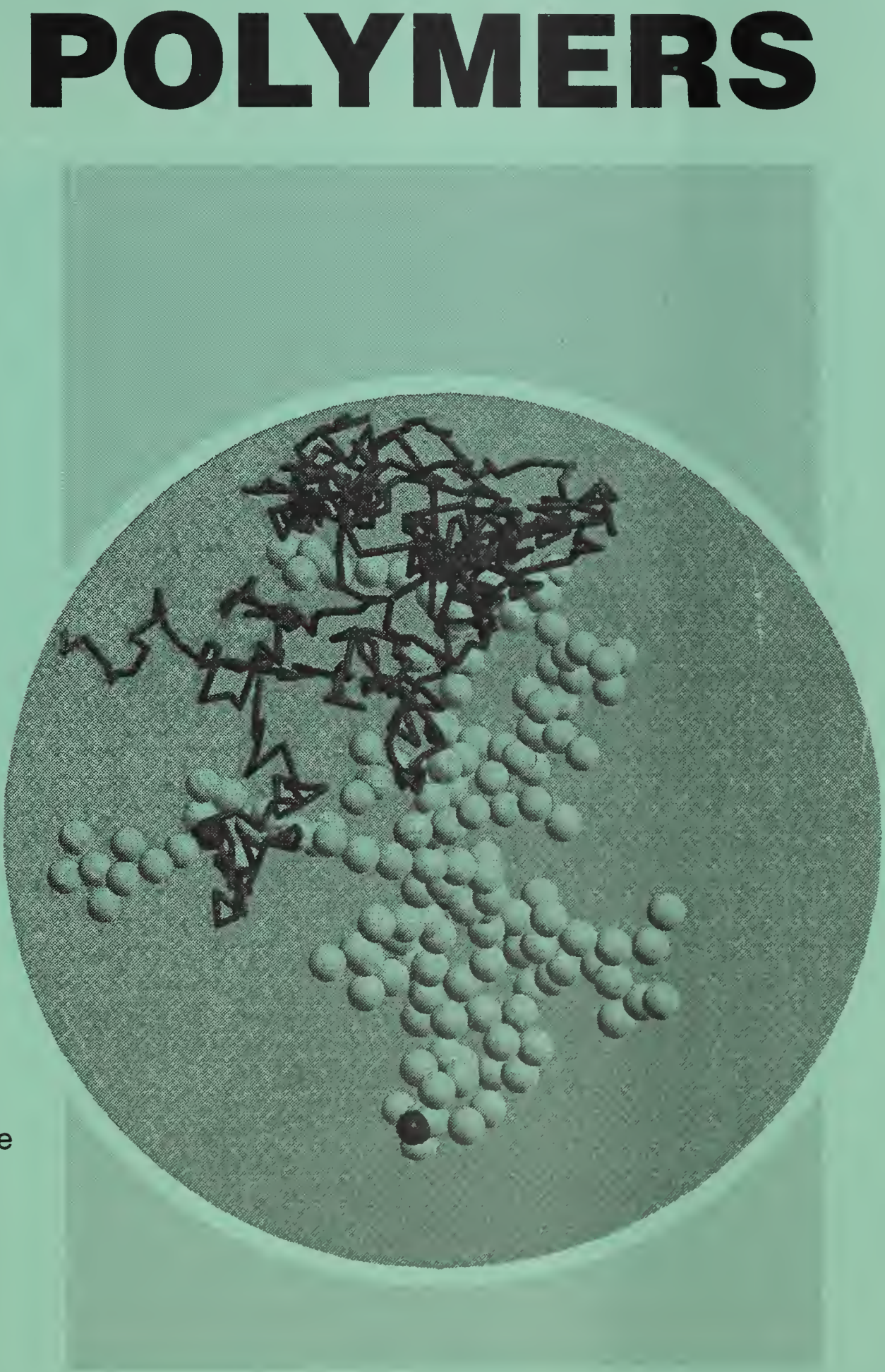

QC 

L.E. Smith, Chief

B.M. Fanconi, Deputy

NAS-NRC

Assessment Panel

April 6-7, 1995

NISTIR 5581

U.S. Department of Commerce

Technology Administration

National Institute of Standards

and Technology

\section{Technical Activities 1994}

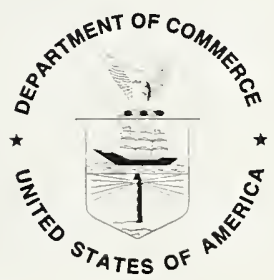

U.S. DEPARTMENT OF COMMERCE

Ronald H. Brown, Secretary

TECHNOLOGY ADMINISTRATION

Mary L. Good, Under Secretary for Technology

NATIONAL INSTITUTE OF STANDARDS

AND TECHNOLOGY

Arati Prabhakar, Director 



\section{POLYMERS DIVISION}

\section{CHIEF}

Leslie E. Smith

Phone: (301) 975-6762

\section{DEPUTY CHIEF}

Bruno M. Fanconi

Phone: (301) 975-6762

\section{GROUP LEADERS}

Electronic Packaging and Interconnects

G. Thomas Davis

Phone: (301) 975-6725

Polymer Blends \& Processing

Charles C. Han

Phone: (301) 975-6771

Polymer Composites

Donald L. Hunston

Phone: (301) 975-6837

Viscoelasticity of Solid Polymers

Gregory B. McKenna

Phone: (301) 975-6752

Polymer Characterization

Bruno M. Fanconi

Phone: (301) 975-6762

Dental \& Medical Materials

Francis W. Wang

Phone: (301) 975-6726 


\section{TABLE OF CONTENTS}

INTRODUCTION $\ldots \ldots \ldots \ldots \ldots \ldots \ldots \ldots \ldots \ldots \ldots \ldots$ iv

RESEARCH STAFF $\ldots \ldots \ldots \ldots \ldots \ldots \ldots \ldots \ldots \ldots \ldots \ldots \ldots$

TECHNICAL ACTIVITIES $\ldots \ldots \ldots \ldots \ldots \ldots \ldots \ldots \ldots$

ELECTRONIC PACKAGING AND INTERCONNECTS PROGRAM . . . . . . . . 1

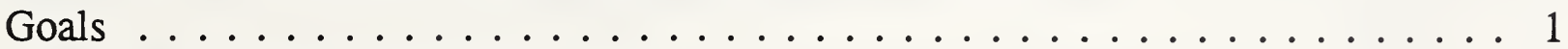

FY-94 Significant Accomplishments . . . . . . . . . . . . . . . 1

Improved Metrology for the CTE of Polymers . . . . . . . . . . . . . 2

Merging Dielectric Measurements from Discrete Frequency Ranges into a

Continuous Spectrum from $\mathrm{DC}$ to $1 \mathrm{GHz} \ldots \ldots \ldots \ldots \ldots$

The Nature of Water Absorbed by Polyimide Electronic Packaging . . . . . . 4 4

Stress in Polyimide Films on Silicon Substrates . . . . . . . . . . . . 4

POLYMER BLENDS AND PROCESSING PROGRAM $\ldots \ldots \ldots \ldots \ldots \ldots$

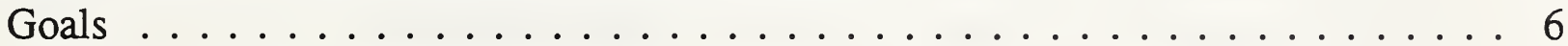

FY-94 Significant Accomplishments . . . . . . . . . . . . . . 6

Polymer Blends Consortium-(Interfacial Modification by Block Copolymers) . . . . 7

Polymer Processing Consortium-(Sensor Development) . . . . . . . . . . . . . . 9

The Effect of Crosslinking on the Phase Behavior of Blends . . . . . . . . . . . 10

Compatibilization of Polymer Blends by Strong Interactions and Reactions . . . . 11

Interfacial Characterization and Phase Separation in Thin Film Polymer Blends

and Block Copolymers . . . . . . . . . . . . . . . . 13

Shear Mixing/Demixing of Polymer Blends . . . . . . . . . . . . . . . 15

Structure Change and Alignment of Block Copolymers Under Shear . . . . . . 16

Synthesis and Characterization of Organic-Inorganic Interpenetrating Polymer

Networks . . . . . . . . . . . . . . . . . . . 17

Pressure Dependence of the Phase Behavior of Polymer Blends . . . . . . . . . . 17

Characterization of Polymer Blends during Processing $\ldots \ldots \ldots 18$

POLYMER COMPOSITES PROGRAM . . . . . . . . . . . . . . . . . . . . . 19

Goals . . . . . . . . . . . . . . . . . . . . . . . . . . . . 19

FY-94 Significant Accomplishments . . . . . . . . . . . . . . . . 20

Liquid Composite Molding: High Speed Flow Behavior and the Development

of Permeability Measurement Techniques and Data . . . . . . . . . . 21

Liquid Composite Molding: Development and Verification of Permeability

Prediction Models . . . . . . . . . . . . . . . . . . . 23

Liquid Composite Molding: Development and Verification of Process

Simulation Models . . . . . . . . . . . . . . . . . . . . . . 26

Liquid Composite Molding: Development of Fiber Optic Sensors for Process

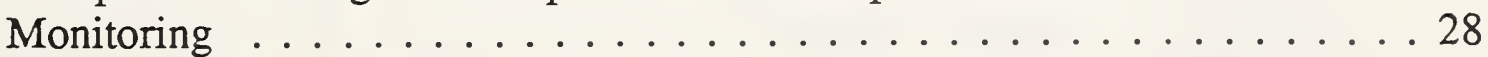

Liquid Composite Molding: Process Monitoring and Control for the

Manufacture of Pre-Ceramic Polymers . . . . . . . . . . 30 
Environmental Durability Studies: Development and Use of the Single Fiber Fragmentation Test to Investigate the Attack of Moisture on the Fiber and Interfacial Strength of E-Glass/Epoxy Samples . . . . . . . . . . 31

Environmental Durability Studies: Comparison of Test Results for Laminated

Composite Samples and Single-Fiber Composite Specimens . . . . . . . . . 32

Environmental Durability Studies: Development of Processing Methods to

Fabricate Urethane Samples . . . . . . . . . . . . . . . . 35

VISCOELASTICITY OF SOLID POLYMERS PROGRAM . . . . . . . . . . 38

Goals . . . . . . . . . . . . . . . . . . . . . . 38

FY-94 Significant Accomplishments . . . . . . . . . . . . . . . . . . . 39

Physical Aging of Polymers . . . . . . . . . . . . . . . . 40

Viscoelastic Behavior of Polymers . . . . . . . . . . . . . 43

POLYMER CHARACTERIZATION PROGRAM . . . . . . . . . . . . . . . . . . 49

Goals . . . . . . . . . . . . . . . . . . . . . . . . . . . 49

FY-94 Significant Accomplishments . . . . . . . . . . . . . . . . . . . 49

Standard Reference Materials . . . . . . . . . . . . . . . . . . . 50

Chromatographic Techniques for Polymer Characterization ......... 51

Structural Characterization of Polymers by NMR . . . . . . . . . . 52

Transport of Small Molecules in Glassy Polymer Matrices . . . . . . . . . . . . . . . 56

Structural Characterization of Polymers by Small Angle X-ray Scattering . . . . . 57

DENTAL AND MEDICAL MATERIALS PROGRAM . . . . . . . . . . . . . . . 60

Goals . . . . . . . . . . . . . . . . . . . . . 60 60

FY-94 Significant Accomplishments . . . . . . . . . . . . . . . . . . . 60

Dental Polymers Designed with Minimal Polymerization Shrinkage, Residual

Vinyl Content, Water Sorption and Oxygen Inhibition . . . . . . . . 61

Dental Composites with Improved Interfaces . . . . . . . . . . . . . . . 65

Improved Adhesive Systems for Bonding to Dentin . . . . . . . . . . . . . . . . 69

Bioactive Polymeric Dental Materials Based on Amorphous Calcium Phosphate

with Remineralization Potential . . . . . . . . . . . . . . . 70

Support for the Biomaterials Integrated Products Industries . . . . . . . . . . 72

Durability and Processing of Dental Ceramic, Metallic and Ceramic-Metal

Materials

THEORY AND MODELING PROGRAM . . . . . . . . . . . . . . . . . . . 75

Theory and Modeling of the Transport Properties of Polymer Solutions . . . . . . 75

Theory and Modeling in Polymer Physics . . . . . . . . . . . . . . 75

Terminally Anchored Chain Interphases . . . . . . . . . . . . . . . . 79

OTHER AGENCY PROGRAMS . . . . . . . . . . . . . . . 81

Goals . . . . . . . . . . . . . . . . . . . . 81

Protection of Archival Records . . . . . . . . . . . . . . . . . . 881

Development of X-ray and Neutron Reflectivity Techniques Using Model 
Elastomer Seal Compatibility for Halon Fire Suppressant Replacements . . . . . . 83 Development and Utilization of Test Methods for Qualification of Passport Laminates . . . . . . . . . . . . . . . . . 86

Monitoring Polymer Processes through Fluorescence Probes . . . . . . . . 86

Scattering from Surfactant Vesicles and Vesicular Dispersions . . . . . . . . 89

OUTPUTS/INTERACTIONS . . . . . . . . . . . . . . . . . 90

Publications . . . . . . . . . . . . . . . . . . . . 90

Technical and Professional Committees: Leadership Activities . . . . . . . . . . 104

Industrial and Academic Interaction . . . . . . . . . . . . . . . . 111

Associated Activities . . . . . . . . . . . . . . . . . . 118

Certain commercial materials and equipment are identified in this paper in order to specify adequately the experimental procedure. In no case does such identification imply recommendation or endorsement by the National Institute of Standards and Technology (NIST) nor does it imply necessarily the best available for the purpose. 


\title{
INTRODUCTION
}

\section{POLYMERS DIVISION}

\author{
Leslie E. Smith, Chief \\ Bruno M. Fanconi, Deputy Chief
}

This year's report is organized according to the programs of the Division and those of its parent Materials Science and Engineering Laboratory. This presentation provides the management context in which we view individual projects in order to make our motivation for the work clearer. We also hope this will encourage our customers to offer comments and advice on ways we can be more effective in our work.

The Polymers Division is responsible for providing standards, measurement methods, and fundamental concepts of polymer science to assist those U. S. industries that produce or use synthetic polymers in essential parts of their business. During this past year we have paid special attention to the ways in which we set priorities for our programs and have begun to explore some more formal methods of measuring the results of our work. Ideally, setting program priorities would lead directly to some measurement of the degree of attainment of the objectives of the programs. In practice, this is seldom the case. Our work rarely leads directly to the development of new technologies or products where the economic effects can be readily estimated. Instead we are charged with helping to provide the measurement basis for our civilian economy - a part of the the technological infrastructure. Measuring the quantitative impact of such work has proved to be exceptionally difficult although the results of some well chosen examples have shown gratifyingly large rates of return on the research investments.

The Division has focussed a majority of its resources on specific industrial sectors. This has allowed us to assess the needs of each industry group and plan a response that is appropriate to our capabilities and role. For example, workshops and discussions with electronics manufacturers have helped defined our program in electronic packaging. As a result, we have concentrated on the role of materials in electronic packaging and have begun to examine some of the measurement methods that provide the critical materials data to evaluate process and product quality or to develop new packaging technologies.

Fiber reinforced polymer composites have tremendous unrealized potential in industries beyond the aerospace industry if manufacturing costs can be reduced and production rates increased. We have worked with the automobile manufacturers for several years on measurement and simulation problems that can aid in the use of composite materials in that industry. This year the Advanced Technology Program began a focussed competition to aid in the introduction of composites into automotive, civil infrastructure and off shore oil applications. 
Our most comprehensive program aims at the producers and proceessors of polymer blends. Here we hope to provide the measurement methods and fundamental understanding that will help producers develop new blend systems more quickly. Resin producers are primarily large, sophisticated, often multi-national companies that want to produce higher value-added materials that also have significant potential volume markets. They need, and can use, very general and fundamental concepts of polymer behavior that affect broad classes of materials.

The Division's program on polymer blends is designed specifically to respond to such needs by providing the scientific basis for phase decomposition after mixing, the effect of compatibilizing additives, and the morphology of blends. These concepts can be used by polymer scientists and engineers to design new blend materials and processes more efficiently. We are working with a consortium of interested companies to help guide our research efforts and to transfer our technology more quickly and efficiently to the companies ready to implement it. We continue to seek more participation in this activity. The consortium has allowed us to consolidate a number of one to one interactions so we can deal with a larger number of customers without additional staff. By effectively training the industry participants to use our facilities and analysis methods we can still retain the flexibility to address the unique questions of each of the companies while continuing a core research program that provides general solutions to the problems they have in common.

The blends program aims to provide support also to the formulators and processors of blends. We have begun activities to develop sensors and models of mixing phenomena that could be applied to process control. This is an ambitious project that will begin with the fundamental principles of polymer phase behavior that we have elucidated in simple shear fields and try to apply them to the complexities of practical mixers and extruders.

In all these programs, collaboration with others continues to be the most effective way to develop and transfer technology. In particular, industry collaborators who know both their business and technical needs are vital to planning and execution. Many of these interactions are briefly described in the section on Industrial and Academic Interactions in the Appendix to this report. 


\section{RESEARCH STAFF}

Amis, Eric J.

amis@micf.nist.gov

Antonucci, Joseph M.

anton@enh.nist.gov

Barnes, John D.

johnbarnes@enh.nist.gov

Bauer, Barry J.

bjbauer@enh.nist.gov

Blair, William R.

willblair@enh.nist.gov

Bowen, Rafael L.*

Briber, Robert J. ${ }^{+}$

Broadhurst, Martin G. ${ }^{+}$ broadhur@micf.nist.gov o Neutron $x$-ray and light scattering

o Polymer characterization

o Strong interactions in polymer/solvent systems

o Viscoelastic behavior of polymers

o Synthetic and polymer chemistry

o Dental composites, cements and adhesion

o Antioxidants

o Gas and vapor transport in polymers

o X-ray scattering

o Computer applications in polymer measurements

o Polymer synthesis

o Neutron, $x$-ray and light scattering

o Dendrimers

o Ultratrace metals speciation

o Polymer analysis by size exclusion chromatography

o Environmental durability of coatings

o Diffusion and absorption of gases in polymeric matrices

o Adhesion

o Dental composites

o Biocompatibility

o Structure and morphology of polymers

o Electron microscopy

o X-ray scattering

o Dielectric measurements

o Piezoelectric and pyroelectric modeling and theory

o Equation of state of polymers

o Ionic conduction

\footnotetext{
* Research Associate

+ Guest Scientist
} 
Bur, Anthony J.

abur@enh.nist.gov

Campbell, Jr., Gordon C. ${ }^{+}$

Carey, Clifton M.*

Chang, Shu Sing ${ }^{+}$

Chiang, Martin Y.

martin@poly3.nist.gov

Chow, Lawrence C.

Dadmun, Mark D.

Davis, G. Thomas

gtdavis@micf.nist.gov o Dielectric properties of polymers

o Fluorescence and optical monitoring of polymer processing

o Piezoelectric, pyroelectric polymers

o Polymeric transducers

o Solid state NMR of polymers

o Off-resonance proton irradiation techniques

- Dental plaque

o Microanalytical analysis techniques

o Phosphate chemistry

o Thermal properties of polymeric and composite materials

o Composite process monitoring

o Electronic packaging materials

o Polymer phase transitions

o Precision electrical and temperature measurements

o Computational mechanics (finite element analysis)

o Strength of materials

o Engineering mechanics of polymer based materials

o Calcium phosphate compounds

o Dental and biomedical cements

o Solution chemistry

o Topical dental fluorides

o Liquid-crystalline polymers

o Polymer Gelation

o Light and neutron scattering

o Electronic packaging

o Polymer crystallization

o X-ray diffraction of polymers

o Polarization distribution

o Piezoelectricity in polymers

- Research Associate

+ Guest Scientist 
DeReggi, Aime S.

dereggi@enh.nist.gov

Dickens, Brian

Dickens, Sabine*

Di Marzio, Edmund A. dimarzio@enh.nist.gov

Douglas, Jack F. shark@enh.nist.gov

Dunkers, Joy P. dunkers@enh.nist.gov

Eanes, Edward D.* dickens@enh.nist.gov

o Polarization-depth profiles in polymers

o Space charge in dielectrics

o Ferroelectric polymers

o Polymeric piezo- \& pyroelectric devices

o Polymer oxidation

o Monomer design and free radical curing

o Automation

o Photoacoustic spectroscopy

o Clinical dentistry

o Dental composites

o Dental polymers

o Statistical mechanics of polymers

o Phase transitions

o Glasses

o Polymers at interfaces

o Theory on polymer solutions and blends

o Polymers at interfaces

o Scaling and renormalization group calculation

o Polymer characterization

o Vibrational spectroscopy of polymers

o Fiber optics spectroscopy

o Process monitoring

o Structure of bones and teeth

o Calcium phosphate compounds as dental materials

o Effects of solution and biological molecules on precipitation of calcium phosphates

o Liposome studies

Eichmiller, Frederick C.*

o Clinical dentistry

o Composites

o Dentin adhesives

Eidelman, Naomi N."

o Atherosclerotic plaque

"Research Associate 
Factor, Bradford J.

factor@enh.nist.gov

Fanconi, Bruno M.

fanconi@micf.nist.gov

Farahani, Mahnaz*

Feng, $\mathrm{Yi}^{+}$

Flynn, Kathleen M.

flynn@enh.nist.gov

Fowler, Bruce O.*

Giuseppetti, Anthony A.*

Glotzer, Sharon C.

glotzer@polygon.nist.gov

Gusler, Gloria M.

gusler@enh.nist.gov

Guttman, Charles M.

guttman@tiber.nist.gov o Reactive blend morphology

o Polymer adsorption

o Neutron reflection, scattering

o Infrared \& Raman spectroscopy of polymers

o Structure of polymers

o Polymer fracture

o Process monitoring of polymer composites

o Radiation chemistry/physics

o Analytical chemistry

o Temperature jump light scattering

o Phase separation kinetics of polymer blend

- Permeability Measurements

- Flow Visualization Experiments

o Infrared and laser Raman structural analysis of calcium phosphates

o Casting of dental alloys

o Titanium alloys

o Theory and simulation of glass-forming materials

o Parallel processing simulations of blends

o Theory and simulation of phase separation kinetics in blends and reactive materials

o Physical aging of polymers

o Crazing of polymers

o Solution properties of polymers

- Size exclusion chromatography

o Semicrystalline polymer chain configurations

o Kinetics of polymer crystal growth

o Diffusion and absorption of pollutant gases by polymeric matrices

Hailer, Arthur W:

o Chemical reactions, chemical analysis

"Research Associate

${ }^{+}$Guest Scientist 
Han, Charles C. han@micf.nist.gov

Hoffman, Kathleen M.*

Horkay, Ferenc ${ }^{+}$

Hunston, Donald L. hunston@micf.nist.gov

Ishikawa, Kunio*

Jackson, Catheryn L. jackscat@enh.nist.gov

Jewett, Kenneth L. jewett@micf.nist.gov

Johnsonbaugh, David S. davjohn@enh.nist.gov

"Research Associate

${ }^{+}$Guest Scientist o Phase behavior of polymer blends

o Phase separation kinetics of polymer blends

o Polymer characterization and diffusion

o Shear mixing/demixing and morphology control of polymer blends

o Static, time resolved, and quasi-elastic scattering of light and neutron

o Synthetic and polymer chemistry

o Adhesion

o Rubber thermodynamics

o Gels

o Adhesion science and technology

o Fracture behavior of polymers

o Processing and failure behaviors of polymer composites

o Flow behavior of dilute high polymer solutions

o Macromolecular-small molecule binding

o Calcium phosphate compounds

o Structure and morphology of polymers

o Transmission and scanning electron microscopy

o Finite-size effects on crystalline melting and glass transition temperatures

o Liquid crystalline polymers

o Microcellular foams morphology

o Redox kinetics of heterogeneous systems

o Organometallic speciation

o Abiotic transformation of metal species

o Analysis of organic mixtures

o Diffusion and absorption of gases in polymeric matrices

o Atomic absorption spectrophotometry

o Microbiology 
Karim, Alamgir

karim@rrdstrad.nist.gov

Keeny III, Spurgeon M. ${ }^{+}$

Kelly, J. Robert

Khoury, Freddy A. ${ }^{+}$ khoury@micf.nist.gov

Kryder, Samuel J. kryder@enh.nist.gov

Kurakawa, Hidenobu ${ }^{+}$

Liu, D.-W.

dwliu@enh.nist.gov

Lowry, Robert E. rlowry@enh.nist.gov

Ly, Agnes M.*

"Research Associate

${ }^{+}$Guest Scientist o Thin film phase behavior of polymer blends

o Morphology of thin polymer blend films

o Block copolymer ordering properties

o Neutron and $\mathrm{x}$-ray reflection, scattering

o Gas plasma sterilization

o Mechanical testing

o Tribiology, dental materials

o Fluorescence studies

o Bioceramics

o Clinical-laboratory test correlation

o Dental ceramics

o Failure analysis; dental prostheses

o Finite element analysis

o Weibull analysis

o Crystallization, structure and morphology of polymers (including polymer blends)

- Analytical electron microscopy of polymers

o Wide angle and small angle x-ray diffraction

o Structure and mechanical property relationships

o Electronic circuit design and construction

o Electronic troubleshooting and repair

o Polymer solution thermodynamics

o Small angle neutron scattering

o Monomer synthesis

o Polymer and polymer composites

o Applications of fluorescence spectroscopy to polymeric systems

o Synthesis of chromophore-labeled polymers

o Clinical dental assistant

o Adhesion measurements 
Macturk, Kenneth S.

kenmac@enh.nist.gov

$\mathrm{Mao}, \mathrm{Yu}^{+}$

Markovic, M.+

Mathew, Mathai*

Maurey, John M.

jrmaurey@enh.nist.gov

McDonough, Walter G. mcdonough@enh.nist.gov

McKenna, Gregory B. greg@micf.nist.gov

McKinney, John E. ${ }^{+}$

Migler, Kalman

kmigler@enh.nist.gov o Polymer composite interfaces

o Adhesion

- Dental plaque

o Microanalytical techniques

o Calcium phosphate chemistry

o Biomineralization (normal and pathological)

o Crystal growth and dissolution kinetics

o Heterogeneous equilibria

o Crystallography

o Calcium phosphate compounds

o Ultracentrifugation

o Rayleigh light scattering

o Osmometry

o Densimetry

o Refractometry

o Intrinsic viscosity

o Processing and cure monitoring polymer composites

o Failure and fracture of polymers

o Polymer composite interfaces

o Failure, yield and fracture of polymers

o Nonlinear viscoelasticity

o Molecular rheology

o Physics of polymer glasses

o Rubber thermodynamics and mechanics

o Mechanics of composites

o Tribiology of dental composites, cements and alloys

o Effects of shear and pressure on phase behavior

o Fluorescence and optical monitoring of polymer processing

o Liquid crystals

o Surface rheology

"Research Associate

${ }^{+}$Guest Scientist 
Misra, Dwarika N.*

Mopsik, Frederick L. fim@enh.nist.gov

Nachlis, Warren L.

Nakatani, Alan I. nakatani@enh.nist.gov

Orts, William J.

Parnas, Richard S. parnas@tiber.nist.gov

Phelan, Jr., Frederick R. fred@poly2.nist.gov

Ranganathan, Sridhar ${ }^{+}$

Ratzker, Menahem B.*

"Research Associate

${ }^{+}$Guest Scientist o Surface chemistry

o Adhesion

o Chemisorption

o Dielectric measurements and behavior

o Automated measurement design

o Computerized data analysis and programming

o Electrical properties of polymers

o Phase behavior \& morphology of polymer blends

o Structure property relationships

o Polymer deformation behavior

o Reactive processing

o Polymer blends and solution properties under shear

o Small angle neutron scattering

o Phase behavior of polymer blends

o Neutron and $\mathrm{x}$-ray scattering

o Polymer morphology and crystallization

o Mechanical properties of polymers

o Flow through porous media with heterogeneous structure

o Surface rheology

o Polymer dynamics

o Evancescent wave optical fiber fluorescence monitoring

o Control of the liquid molding process

o Resin transfer molding: modeling and processing studies

o Viscoelastic flow modeling

o Flow in porous media

- Computerized modeling of fluid flow

o Process simulation models for composites

o Structure-based property predictions for porous media

o Electrodeposition 
Reed, Benjamin B.*

Roth, Steven C.

sroth@micf.nist.gov

Rupp, Nelson W.*

Sakurai, Shinichi ${ }^{+}$

Sanin, Norman D.*

Schen, Michael A. schen@micf.nist.gov

Schultheisz, Carl R. carl_s@enh.nist.gov

Schutte, Carol L. schutte@enh.nist.gov o Synthetic and polymer chemistry

o Polymerization expanding monomers

o Piezoelectric polymer transducers-fabrication and applications

o Vacuum deposition of metals

o Calibration of polymer transducers

o Microcomputer interfacing

o Fluorescence measurements

o Clinical dentistry

o Amalgams

o Direct golds

o Composites

o Microleakage

o Dentin adhesives

o Polymer blends

o Equilibrium and kinetics of phase separation

o Light scattering

o Topical dental fluorides

o Calcium phosphate cements

o Non-linear optical polymer

o Optical spectroscopy

o Micoelectronics packing materials

o Photonics

o Coefficient of thermal expansion

o National technology roadmaps in electronics

o Failure of composites

o Experimental mechanics

o Torsional dilatometry

o Physics of polymer glasses

o Surface chemistry

o Polymer composite interfaces

"Research Associate

${ }^{+}$Guest Scientist 
Sieck, Barbara A.*

Skrtic, Drago*

Smith, Leslie E.

smith@micf.nist.gov

Stansbury, Jeffrey W. stansbury@enh.nist.gov

Stephenson, Maurice A.S.

Takagi, Shozo*

Tesk, John A. tesk@micf.nist.gov o Calcium phosphate compounds

o Chemical analysis

- Calcium phosphates as dental materials

o Lipsome studies

o Adsorption of polymers

o Polymer degradation reactions

o Synthetic chemistry

o Polymers and polymer composites

o Polymerization expanding monomers

o Nuclear magnetic resonance

o Synthetic and polymer chemistry

o Composites development

o Catalysis

o Crystallography

o X-ray diffraction

o Calcium phosphate compounds

o Biomaterials-industrial relations

o Bond strength characterization

o Casting of alloys

o Strength of dental systems

o Thermal expansion and properties of dental materials

o Finite element studies

o Porcelain-metal systems

o Weibull analysis

o Gas plasma sterilization

o Processing of dental ceramics, effects of

Tomazic, Branko*

o Atherosclerotic plaque

o Biological calcium phosphate compounds

Tung, Ming A.*

o Biological solution chemistry of calcium phosphate compounds

o Equilibrium studies

"Research Associate 
VanderHart, David L. vdhart@enh.nist.gov

Van Zanten, John $\mathrm{H}$. johnvz@enh.nist.gov

Verdier, Peter H. verdie@enh.nist.gov

Vogel, Gerald L.*

Waldron, William K. waldron@enh.nist.gov

Wallace, William E. wallace@enh.nist.gov

Wang, Francis W. fwwang@micf.nist.gov

Winnert, Laurie A.* o Measurement of orientation in polymer fibers and films

o Solid state NMR of polymers

o Measurement of polymer morphology at the $2-50 \mathrm{~nm}$ scale

o Structure of asphalt cements and concretes

o Pulsed field gradient NMR

o Complex fluids

o Polymer interfaces

o Scattering of light, neutrons \& x-rays

o Biophysics

o Interfacial phenomena

o Scanning probe microscopy

o Polymer solution properties

o Polymer chain dynamics in solution

o Statistical analysis of data

o Error estimation

o Computer simulation of polymer chain dynamics

o Dental plaque, biological solution chemistry of calcium phosphates

o Microanalytical techniques

o Nonlinear viscoelasticity

o Mechanics and thermodynamics of rubber

o Surface and interface behavior

o Ion beam and electron spectroscopies

o X-ray and neutron reflectivity

o Photophysics and photochemistry of polymers

o Fluorescence spectroscopy

o Thermodynamic and frictional properties of macromolecules

o Glass-ceramics

"Research Associate

${ }^{+}$Guest Scientist 
Woerdeman, Dara ${ }^{+}$ levy@tiber.nist.gov

Wu, Wen-li

wenli@enh.nist.gov o Permeability

o Composite process monitoring

o Evanescent wave optical fiber fluorescence monitoring

o Neutron and $\mathrm{x}$-ray scattering and reflectivity

o Electron microscopy

o Mechanical behavior of polymers and composites

o Polymer surfaces and interfaces

${ }^{+}$Guest Scientist 


\section{TECHNICAL ACTIVITIES}

\section{ELECTRONIC PACKAGING AND INTERCONNECTS PROGRAM}

\section{$\underline{\text { Goals }}$}

The technology of integrated circuits has advanced to the point where the performance of systems in which they are incorporated is limited by the properties of the materials in which they are encapsulated and the performance of the interconnections between components in the system. Polymers have become the material of choice for packaging and interconnects in most applications because of their low dielectric constant, light weight, and ease of processing despite some problems with moisture absorption and unfavorable coefficients of thermal expansion. The pressure of competition in consumer electronics has reduced the time from design to production of new electronic packages to only a few months with further reductions anticipated in the near future. This has placed increased demands on the need for reliable data on the properties of materials over a wide range of processing and use conditions to be used in mathematical models for the design, manufacture and reliability assessment of packaged integrated circuits.

This program addresses the needs of the electronic industry for reliable data on the physical properties of polymers and their variation with processing parameters. The approach taken is not focussed on generation of data, however. The emphasis is on refinement of existing measurement methods, or development of improved methods in cooperation with industry, academia, and other government agencies, especially for polymers in the form in which they are used. The focus is on the properties of thin films, both free standing and in juxtaposition with dissimilar materials. Current measurement programs are concerned with thickness direction coefficient of thermal expansion, dielectric properties from $\mathrm{DC}$ to $1 \mathrm{GHz}$, and the assessment of residual stress in polymeric films. In addition, the role of moisture in the development of cracks in polymeric encapsulants subjected to solder reflow temperatures is being investigated from a fundamental standpoint.

\section{FY-94 Significant Accomplishments}

- A workshop involving 108 representatives from the electronics industry, academia and other government agencies was held to assess the needs of the electronics industry for metrology and data in packaging and interconnects.

- A cooperative program on the measurement of thermal expansion of thin films in the thickness direction has shown the limitation of commercial thermal mechanical analyzers for such measurements which prompted the design and construction of a capacitance cell for measuring thermal expansion.

- Instruments have been obtained for measuring dielectric properties at frequencies between $10 \mathrm{kHz}$ and $1 \mathrm{MHz}$, a region in which our instrumentation was deficient. An 
instrument for deducing residual stress from wafer deformation has been obtained as well as an instrument which employs a prism coupler for measuring the refractive index and thickness of polymer films.

\title{
Improved Metrology for the CTE of Polymers
}

\author{
M.A. Schen, F.I. Mopsik, S.C. Roth and G.T. Davis
}

\section{Objectives:}

Using a commercially available thermal mechanical analyzer (TMA) and a NIST designed capacitance cell, the objectives are to improve and develop techniques and protocols to accurately measure the out-of-plane coefficient of thermal expansion (CTE) of polymer thin films typically used in microelectronics packaging.

\section{Accomplishments:}

Today's microelectronics products rely heavily on the use of polymeric materials whether to package and protect the integrated circuit or to manufacture printed wiring boards and assemblies. Measurements and data for out-of-plane CTE are used by packaging design engineers to assure reliable products. Results of the May 1994 NIST, Semiconductor Research Corporation (SRC), Institute for Interconnecting and Packaging Electronic Circuits (IPC) and Optoelectronics Industry Development Association jointly cosponsored workshop on Metrology and Data for Microelectronic Packaging and Interconnection, (NISTIR 5520) identify dimensional stability of materials, especially polymers, as a critical technical challenge for the industry. Development of in-situ, in-use, and in-process metrology is also highlighted as a cross-cutting issue.

Although not designed for measuring the very small out-of-plane (z-axis) strains anticipated in polymer thin films during manufacture or use, the TMA continues to see widespread use in the electronics industry for measuring CTE due to the convenience of the measurement and the ready availability of the instrumentation. To improve upon in-place industry standards, NIST is working with IBM, Digital Equipment Corp., Microelectronics and Computer Technology Corporation, University of Maryland/CALCE, Cornell University, University of Texas/Austin, Purdue University, Naval Surface Warfare Center at Crane, and the Semiconductor Research Corporation to develop a TMA testing methodology that utilizes silicon single crystal as a working standard against which polymer measurements are evaluated. Using round-robin methods, the team has evaluated uncertainties in the technique and is progressing toward establishing a point-by-point correction for specimen data.

In a parallel and complementary effort, NIST has successfully developed and demonstrated a dimensionally stable, nearly constant electrode area capacitance cell that can accurately measure the thermal expansion of silicon single crystal over a broad temperature range. A key requirement for obtaining accurate data to $1 \mathrm{ppm}$ in total length is the need to maintain very precise temperature control and account for variations in the dielectric constant of the atmosphere between the electrodes that can vary with humidity and atmospheric pressure. 
Viewed to be a much superior technique for measuring z-axis CTE, the technique is being applied to the characterization of polyimide (PI) films used in microelectronics manufacturing. In capacitance experiments using $50 \mu \mathrm{m}$ anisotropic PI films, the new technique shows a change in CTE from $63 \mathrm{ppm} /{ }^{\circ} \mathrm{C}$ at $50{ }^{\circ} \mathrm{C}$ to $92 \mathrm{ppm} /{ }^{\circ} \mathrm{C}$ at $135{ }^{\circ} \mathrm{C}$. These measurements also demonstrate limits in measurements of linear dimension over time for PI specimens. The technique was able to document changes in material dimensions due to creep or changes in the material itself. Results such as these demonstrate the possible utility of the capacitance technique to conduct in-situ creep measurements on extremely thin polymer films.

\section{Merging Dielectric Measurements from Discrete Frequency Ranges into a Continuous Spectrum from DC to $1 \mathrm{GHz}$}

F.I. Mopsik, A.S. DeReggi, S.C. Roth and G.T. Davis

\section{Objectives:}

Develop measurement techniques to accurately measure the dielectric performance of thin film polymeric insulators used in electronics applications.

\section{Accomplishments:}

Insulation used in electronics applications must meet high performance standards over a very wide range in frequency. This insulation can often set the performance limits for devices. Some of the most desirable polymers are often available only as thin films, creating additional measurement problems at higher frequencies. Current practice, even at $1 \mathrm{MHz}$, has sufficient uncertainty to create problems for the semiconductor industry. While low frequency techniques developed in this laboratory can cover the range from $\mathrm{DC}$ to $10 \mathrm{kHz}$, higher frequencies require different methodologies.

To develop reliable techniques covering the entire frequency spectrum, four-terminal precision LCR meters covering the frequency range from $100 \mathrm{~Hz}$ to $30 \mathrm{MHz}$ have been added to existing Time-Domain instrumentation and a $1 \mathrm{MHz}$ to $1 \mathrm{GHz}$ Impedance Analyzer. The instruments have been analyzed for measurement uncertainties and initial calibrations verified. A sample holder has been designed for use in both three- and four-terminal configurations with minimal error so that only one sample needs to be used from DC to up to $30 \mathrm{MHz}$. Preliminary design studies have been completed on requirements for the sample configurations usable to $1 \mathrm{GHz}$. Initial data taken on a polyimide film have shown the limits of the commercial instrumentation. Besides shifts in the dielectric constant directly attributable to instrumental calibration, the loss values proved to be quite unreliable toward the high portion of a given instrument's frequency range. However, these results also have shown that it should be possible to get reliable dielectric constant and loss data to a relative error of better than $0.1 \%$ just above $10 \mathrm{KHz}$ declining to $1 \%$ at $1 \mathrm{GHz}$ on thin film materials. 


\title{
The Nature of Water Absorbed by Polyimide Electronic Packaging
}

\author{
Brian Dickens and G.T. Davis
}

\section{Objectives:}

The objectives are to use near infrared spectroscopy (NIR) and other techniques such as gravimetry to follow the absorption and desorption of water in polyimides and polyimide composites. A further objective is to deduce the mechanism by which the absorbed water is held in the structure.

\section{Accomplishments:}

Pop-corning refers to the formation of moisture-induced defects in polymeric packaged electronics when subjected to rapid heating during solder reflow operations and is a prevalent industrial problem. The mechanism by which the presence of moisture leads to failure is not known but eradication of the phenomenon would greatly benefit the electronic packaging and assembly industry. This work aims towards understanding the absorption of water in electronic packaging components and is intended, when taken together with data and experience provided by industry, to help pinpoint the root causes of pop-corning.

Water absorption by a material is the result of the availability of water and the presence of chemical groups in the material to which water can hydrogen-bond. The strength of the hydrogen bond of water to these chemical groups can be followed in the near infrared over the range 5160 to 5300 wavenumbers. The stronger hydrogen bonds absorb NIR radiation at the lower wavenumbers and have lower absorptivities. Further, because the NIR is weakly absorbed, relatively thick specimens ( 25 to several hundred micrometers) can be used. The absorptivity of the hydrogen bond and its location in the above frequency range have been established for hydrogen bonds from water to a variety of materials, including simple polyimide films. Experiments are underway to provide similar information on the hydrogen bonds between water and epoxy molding compounds as well as unsilanized silica-filled composites of both polyimide and epoxy. These measurements will provide information on the relative rates of hydration of the polymers and the silica.

\section{Stress in Polyimide Films on Silicon Substrates}

\author{
A.S. DeReggi, Sigfried Bauer ${ }^{1}$, S.C. Roth, B. Dickens and G.T. Davis \\ ${ }^{1}$ Heinrich Hertz Institut fur Nachrichtentechnik, Berlin, Germany
}

\section{Objectives:}

Using commercial instrumentation or in-house developed capacitive, integrated piezoresistive, or optical lever techniques, the objectives are to obtain stress or strain data and to relate these data to structural, conformational or morphological properties of the film before and after curing as well as the thermal properties of the cured film relative to those of the substrate. 


\section{Accomplishments:}

A commercial instrument designed for factory-floor measurement of thin film stress on silicon wafers has been procured. The instrument is of the linearly-scanning, optical-beam type, where the deflection of the reflected beam at a general point along the scanned line sampling a wafer diameter is related to the local slope of the wafer, and stress is calculated by software from the average curvature of the wafer surface determined from the scanning data. This instrument has been used to determine the spin-coating conditions required to obtain uniform thickness of the coating in the thickness range $1-10 \mu \mathrm{m}$ as gaged by the uniformity of the curvature changes induced by curing. It was established that spinning a substrate at constant angular velocity tends to make worse any initial deviation from a uniform mass distribution of a liquid coating the substrate in the radial direction and furthermore that spinning has a negligible effect in homogenizing the mass distribution in a tangential direction. It is concluded that spin-coating is a poor method of producing uniform films except for thicknesses where surface tension or molecular forces are the thicknessdetermining factors rather than inertial forces. Methods for improving the thickness uniformity of spin-coated films are being devised. The instrument has also been used to determine the effect of the initial curvature of a wafer on the stress determined by the instrument. Preselection of wafers has been determined to be desirable to avoid a change in sign of the wafer curvature induced by film curing. The instrument has been used to measure the change in curvature during curing cycles for a standard and widely used, electronics-grade polyimide and a developmental, low in-plane- thermal-expansion polyimide developed to minimize stress induced by cooling from the curing temperature of approximately $350^{\circ} \mathrm{C}$. For the standard polyimide, the dominant stress develops during cooling after curing whereas for the low-expansion polyimide the dominant stress is transitory and occurs during the heating part of the cure cycle in a $30^{\circ} \mathrm{C}$ temperature interval corresponding to the solvent loss. Low-stress qualities of polyimides in the in-plane direction are obtained by making the thermal expansion/contraction coefficient and other properties highly anisotropic, the expansion out-of-plane being greater than in-plane. The anisotropy depends on the degree of orientation imparted by the film preparation procedure. A new thermal pulse technique has been developed for measuring out-of-plane thermal diffusivity and used to make preliminary measurements on standard and low stress polyimides. Good agreement with manufacturer's data has been found for the standard (isotropic) polyimide and 20 per cent higher diffusivity has been found for the low stress polyimide. 


\section{POLYMER BLENDS AND PROCESSING PROGRAM}

\section{Goals}

The goal of the Polymer Blends and Processing Program is to assist the polymer industry by developing fundamental understanding, measurement techniques, data, and process control methods for mixing processes and control of morphology of polymers and polymer blends.

Processing is one of the most important steps in the manufacturing of plastic products. Due to more stringent requirements in the properties and dimensional stability for new applications and in new product development, together with the complex nature of multiphase processing in polymer blends/alloys, a more scientifically disciplined approach in process design and control has become the key issue in gaining competitiveness in the 1990's. Although the growth in the total annual production and in new product development has remained strong in the late 1980's and early 1990's for polymer blends/alloys (annual growth rate of $8-10 \%$, and a total world market of 24 billion pounds for 1994, according to the prediction by Chemical Week, May 1990), the challenge of responding to specific customer/market needs while reducing the new-product development cycle, can only be met through a more scientific and systematic approach, instead of the trial-and-error approach mostly used in the 80's (see Plastics Technology, June 1992).

Research in this program is aimed at working with industry for a more scientifically disciplined approach in process design and control for new blends materials in order to achieve a more efficient response to customer/market needs and a shorter new-product development cycle. Specific topics are focused on the areas of: (I) characterization of materials at various stages, (ii) mixing/demixing processes, (iii) compatibilization and stabilization through interfacial modification, (iv) property measurements, and (v) technology transfer.

Experimental and theoretical studies relevant to polymers and polymer blends are actively pursued. Measurement techniques employed include small angle neutron scattering (SANS), time resolved SANS, in-situ shear SANS, time resolved temperature-jump light scattering (TJLS), shear light scattering, conventional elastic light scattering, dynamic light scattering, neutron and X-ray reflectivity techniques, atomic force, optical and electron microscopies, and various kinds of fluorescence monitoring and measurements. A new shear device with both time resolved light scattering and phase contrast microscopy/image analysis detection system is also available.

\section{FY-94 Significant Accomplishments}

- Two new projects were initiated with industrial members of the NIST/Industry

Consortium on New Measurement Technology for polymer Processing.

Evaluation of an in-line fluorescence confocal sensor for measuring 
temperature profiles in extruded resins is underway with DuPont Co and development of a sensor to monitor fluorescence and pressure simultaneously is being carried out with Dynisco.

- Under the NIST/Industry Consortium on Polymer Blends and Alloys the morphological control of immiscible polystyrene/polybutadiene blend, by the addition of polystyrene-polybutadiene block copolymer as compatibilizer is studied by SANS and time resolved light scattering methods. In-situ characterization of shear mixing and development of scattering and microscopy characterization techniques on the mixer/extruder line are underway.

- A new light scattering/optical microscope detection system has been built and adapted to a shear rheometer (cone and plate or plate and plate geometries) for in situ, real time studies of shear mixing and de-mixing of polymer blends. The instrument permits simultaneous characterizations in both real space (optical microscope) and reciprocal space (light scattering).

- Novel surface roughening and pattern formation have been observed in polystyrene/poly(vinylmethylether) and in poly(methylmethacrylate)/polycarbonate thin films on silicon substrates. The phase separation process in these blends is accompanied by a unique pattern evolution at the free air boundary of the film.

- TEM results on sheared and quenched block copolymer of polystyrene$\mathrm{d}_{8} /$ polybutadiene/polystyrene- $\mathrm{d}_{8}$ indicated a high shear rate induced change in the packing symmetry of the microphase separated cylinders akin to "martensitic transformations" observed in metals. This result implies that theories developed for atomic scale structural changes in metals may be more broadly applicable to the block copolymer class, on a meso-size scale.

- Through control of polymerization conditions wide variations in morphology, interfacial width, and thermal stability have been obtained on a series of interpenetrating polymer networks in mixtures with orthosilicates.

\section{Polymer Blends Consortium-(Interfacial Modification by Block Copolymers)}

C.C. Han, C.L. Jackson, A.I. Nakatani, J.F. Douglas, B. Bauer, L. Sung ${ }^{1}$, A. Karim and D.B. Hess ${ }^{2}$

${ }^{1}$ University of California, Santa Barbara, CA

${ }^{2}$ National Physical Scientist Consortium, Las Cruces, NM

Objective:

The objective is to conduct a thorough scientific study of the compatibilization of polymer 
blends based on block copolymers using a model system. This will include gaining insights into the kinetics of phase separation, as studied by light scattering, and the ultimate morphology of the blend, studied by neutron scattering and optical and transmission electron microscopy.

\section{Accomplishments:}

SANS Study of the Equilibrium Phase Behavior of PS/PB/PS-PB Blends

Small angle neutron scattering measurements have been conducted to investigate the effect of polystyrene- $\mathrm{d}_{8} /$-polybutadiene diblock copolymer (PSD-PB) content on the phase behavior of a low-molecular-weight polystyrene- $\mathrm{d}_{8} /$ polybutadiene (PS/PB) mixture. SANS results include general features of the scattering curves as a function of temperature and copolymer content. For intermediate to high copolymer content (greater than $20 \mathrm{wt} \%$ ), a pronounced peak in the scattering curve is observed at all temperatures. The peak intensity, $S\left(q^{*}\right)$, increases as temperature decreases for all samples, and no sharp change in the temperature dependence of $\mathrm{S}\left(\mathrm{q}^{*}\right)$ is observed. The position, $\mathrm{q}^{*}$, increases with increasing copolymer content and is weakly dependent on temperature. The analysis of the temperature and composition dependence of the peak intensity and peak position is being carried out.

Time Resolved Light Scattering and Microscopic Investigation of the Kinetics of Phase Separation and Structure Formation of PS/PB Blends with/without Interfacial Modifier

The phase separation kinetics and morphology of binary polymer blends of polystyrene-d8 $(\mathrm{PSD}, \mathrm{Mw}=1000 \mathrm{~g} / \mathrm{mole}$ ) and polybutadiene $(\mathrm{PB}, \mathrm{Mw}=5000 \mathrm{~g} / \mathrm{mole}$ ) upon addition of a compatibilizing agent (PSD-PB diblock copolymer, $\mathrm{Mw}=10,000 \mathrm{~g} / \mathrm{mole}$, symmetric) have been studied using temperature jump light scattering (TJLS) and phase-contrast optical microscopy techniques. The phase diagram of the binary blend was determined in the range of 40 to $90 \mathrm{wt}$.\% PS and the critical composition of the blend was $75 / 25$ by weight. The critical temperature was $\sim 51{ }^{\circ} \mathrm{C}$. Upon addition of a small amount of diblock copolymer $(3 \%)$, the kinetics of phase separation was significantly slower and the phase boundary shifted to lower temperature by about $5{ }^{\circ} \mathrm{C}$. This is expected based on other systems described in the literature, since the block copolymer effectively reduces the interfacial tension between the demixed phases and concentrates at the interface. The optical microscope is unable to resolve the early stages of phase separation in the $75 / 25$ "critical" blend, because the size scale is smaller than the resolution of the optical microscope. Offcritical blends are easily observed, however, and addition of block copolymer to a 70/30 blend was examined in detail. The morphology formed in the two-phase region is smaller in size as block copolymer was added, and observations could be made up to about $5 \%$ block copolymer before the resolution limit was reached. Further studies using transmission electron microscopy, which has much higher resolution, are in progress, in addition to plans for small-angle neutron scattering (SANS) experiments. Furthermore, a theory of blend stability upon addition of either an asymmetric or symmetric diblock copolymer has also been developed, in parallel to the experimental work. This system was chosen by the Polymer Blends Consortium as a model system to study, and an update of the work has been 
presented to the Consortium.

\title{
Polymer Processing Consortium-(Sensor Development)
}

\author{
A.J. Bur, K. Migler, S.C. Roth, R.E. Lowry and D.S. Johnsonbaugh \\ Objective:
}

This program is being carried out within the framework of a consortium agreement with members of the polymer processing industry. The goal of the consortium is to develop sensor technology to measure, nondestructively and in real-time, important processing parameters. The sensor technology is based primarily on optical measurement methods which are designed to monitor those parameters deemed important by polymer processors, namely, rheology and temperature of the processed resin.

\section{Accomplishments:}

Accomplishments during the past year are: in-line temperature measurements during extrusion comparing temperatures obtained from fluorescence observations with those obtained by IR radiometry, measurement of the pressure coefficient for fluorescence production from temperature sensitive dyes and using this coefficient to correct fluorescence temperature measurements, completion of model calculations for two methods designed to obtain temperature gradients using fluorescence, successful synthesis of pyrene tagged poly(methyl methacrylate), the development of models to describe and predict the behavior of a fiber optic sensor used to monitor polymer injection molding, and successful in-line testing of a polarizing optics sensor for measuring fluorescence anisotropy.

Fluorescence spectroscopy and optical fiber cables, which are inserted into process equipment using existing instrumentation ports, are the main tools that are being exploited to attain the measurement objectives. Temperature sensitive fluorescent dyes which are doped into the polymer resin at parts per million concentration are used to acquire actual resin temperatures as opposed to the processing machine temperature which is obtained by using thermocouples.

The effect of shear heating on resin temperature has been monitored during extrusion by measuring the excimer fluorescence radiation from the temperature sensitive dye, 1,3,bis(1-pyrene) propane doped into poly(methyl methacrylate). Shear heating was effected by increasing the screw rpm. The optical sensor, an IR radiometer, and a thermocouple were positioned in identical $1 / 2$ inch standard instrumentation ports. Both fluorescence and IR radiometer temperatures increased by more than $10^{\circ} \mathrm{C}$ upon change from 20 to $40 \mathrm{rpm}$, but the thermocouple temperature rose only by $2^{\circ} \mathrm{C}$ verifying the assumption that the thermocouple monitors the machine temperature and yields only small changes in temperature because of the large thermal mass of the machine.

Temperature gradients are an important processing parameter, particularly, in the case of extrusion for which shear heating can cause large temperature gradients and overshoots above 
the machine temperature set point. The possibility of using temperature sensitive dyes to measure temperature gradients non-destructively has been the focus of model calculations to describe two potential techniques. Model calculations of two sensor designs have been carried out and show that the technique which employs confocal optics is capable of temperature and spatial resolutions of $1{ }^{\circ} \mathrm{C}$ and $1.5 \mathrm{~mm}$ respectively using 1,3-bis(1-pyrene) propane. Laboratory experiments to confirm this are underway.

An optical sensor which utilizes polarizing optics has been designed, constructed and successfully used to obtain real-time measurements of fluorescence anisotropy during extrusion of polycarbonate doped with perylene. Since anisotropy is a measure of molecular orientation, it reflects the magnitude of applied stress to the resin during extrusion. Anisotropy measurements, in addition to pressure and temperature measurements, can be used to describe the rheology of the extrusion process.

In some cases, it is desirable to tag the fluorescent moiety to the main chain of the polymer in order to obtain information about molecular orientation. Pyrene tagged poly(methyl methacrylate) has been synthesized for this purpose. Measurement of the production of excimer radiation from overlapping pyrene rings as a function of applied stress is the objective for this material.

Modeling the response to changes in the resin of a fiber optic sensor, has been carried out. The sensor was used for light transmission and fluorescence measurements during injection molding of polyethylene, polypropylene and polystyrene. The data were analyzed by constructing a model of light transmission and fluorescence detection in crystallizing or glass forming resins. The calculations yield information about the time of crystallization and glass formation, the thermal resistance at the resin/mold interface, and volume relaxation in a quenched glass forming resin.

\section{The Effect of Crosslinking on the Phase Behavior of Blends}

B.J. Bauer, Da-Wei Liu, R.M. Briber ${ }^{1}$ and Xiao-Du Liu ${ }^{1}$

${ }^{1}$ University of Maryland, College Park, MD

\section{Objectives:}

It is necessary to understand the effects of crosslinks on the morphology of interpenetrating polymer networks (IPNs) since morphology controls all of the important physical properties. IPNs are a commercially important type of blends that would benefit from such understanding.

\section{Accomplishments:}

Crosslinking has been shown to destabilize polymers in conventional interpenetrating polymer networks (IPNs), while IPNs in which grafting between the components is introduced, miscibility is enhanced. Linear chains in polymer networks are seen to diminish in size until 
they collapse into aggregates, even at extremely low linear chain concentrations.

Small angle neutron scattering has been used to measure the conformation of linear polymer chains at dilute concentrations trapped in a matrix of crosslinked polymer. Three regimes are found depending on the length of the linear chain, $\mathrm{Nl}$, with respect to the mesh size of the network, Nc. When $\mathrm{N} l<\mathrm{Nc}$ the radius of gyration of the linear chain does not change from that observed in the uncrosslinked melt. When $\mathrm{Nc}<\mathrm{N}$, the size of the linear chains shrinks which has been predicted for isolated polymer chains trapped in a field of random obstacles. When $\mathrm{Nc}<<\mathrm{Nl}$ the linear chains are observed to segregate.

\section{Compatibilization of Polymer Blends by Strong Interactions and Reactions}

C.C. Han, B. Factor, A. Karim, G. Merkle ${ }^{1}$, F.I. Mopsik, Y. Feng ${ }^{2}$, W. Nachlis and H. Yoon ${ }^{3}$

${ }^{1}$ Institut fur Makromoleculare Chemie, Freiburg, Germany

${ }^{2}$ University of Connecticut, Storrs, CT

${ }^{3}$ University of Wisconsin, Madison, WI

\section{Objective:}

The objective is to characterize hydrogen bonding interactions, ionic complexation and transesterification reactions as methods for compatibilization of polymer blends.

\section{Accomplishments:}

Several new approaches have been investigated for control of binary polymer blend phase behavior through either strong interactions or transesterification reactions. In the studies, changes to both the phase diagram and the kinetics of phase separations have been characterized for several different binary blends. Strong interactions may be introduced by chemically modifying segments to form sites for complexation or hydrogen bonding. It was shown that the introduction of a small number of interactive sites can dramatically change phase behavior in blends which are ordinarily incompatible. Another way to change the phase behavior is through transesterification reactions at high temperatures which result in either an exchange of segments between the different polymers or the grafting of one polymer onto the other. Transesterification induces compatibility in otherwise incompatible mixtures, leading to a homogeneous phase at high temperatures. At intermediate temperatures, by contrast, a microdomain morphology with a broad interphase forms. A final aspect of this project examines compatibilization by the aforementioned mechanisms in ultra thin film polymer films cast on silicon substrates. In this case it is observed that the transesterification process produced a copolymer layer which gradually forms at the interface close to the substrate. For a bilayer of two polymers which interact through strong interactions, mixing at the interface due to the complexation between the two components is found. Details on specific tasks are given below.

Control of Phase Behavior Through Hydrogen Bonding 
Hydrogen bonding in blends of polybutylmethacrylate (PBMA) and deuterated polystyrene (d-PS) was achieved by introducing a small amount perhexafluoro-2-hydroxyisopropyl- $\alpha$ methylstryene as a co-monomer in the d-PS polymerization. The resulting polystyrene, denoted d-PSOH, contains hydroxyl groups which have nearly the same acidity as phenol. In the d-PSOH/PBMA blend, Pearce et al. [1] have shown the presence of hydrogen bonding between the d-PSOH hydroxyl and the PBMA carbonyl groups. A small amount $(1.2 \mathrm{~mol}$ $\%)$ of the perhexafluoro-2-hydroxyisoproyl groups dramatically affects the phase behavior. The d-PSOH/PBMA blend is miscible within a limited temperature range whereas the unmodified blend using identical molecular weights is immiscible. Small angle neutron scattering has been employed to study the kinetics of phase separation in the d-PSOH/PBMA blend. These experiments revealed an unusual growth behavior controlled by two separate length scales in hydrogen bonded blends. This might result in a domain structure which is different from the interconnected phases seen in classical spinodal decomposition, and there is a possibility that this might be useful for new materials.

\section{Control of Phase Behavior Through Complexation}

Ionic complexes provide another means to modify the interactions between immiscible polymers. This study focussed on how the nature and strength of complexation affects blend morphology and phase behavior. The blend consists of lightly sulfonated polystyrene ionomers with poly $\left(\mathrm{N}, \mathrm{N}^{\prime}\right.$-dimethylethylene sebacamide), a methylated Nylon. Because the polyamide exhibits no hydrogen bonding, as a result of substitution of the methyl group for the amide proton, it has a relatively low melting temperature $\left(\sim 75^{\circ} \mathrm{C}\right)$ and is slow to crystallize. Time resolved light scattering was undertaken to examine the phase separation process in this blend, and found that there is a temperature range where phase separation can occur but is limited by the complexation interactions. It appears that resulting morphology is influenced by the level of sulfonation and the type of counter cation; this will be a subject of investigation in the FY95.

\section{Structural Stabilization of Through Transesterification Reactions}

Small angle neutron scattering was employed to determine how the morphology of two types of mixtures, polycarbonate (PC) with a noncrystallizable polyester copolymer and PC with polymethylmethacrylate (PMMA), can be controlled by choosing the appropriate thermal treatments. At high temperatures, transesterification results in the exchange of segments in the PC/polyester blend, forming a random copolymer. By contrast, PC chains ends graft onto the PMMA backbone in the PC/PMMA blend. For both mixtures, annealing below $170^{\circ} \mathrm{C}$ results in large scale phase separation, just as for ordinary immiscible blends. However, when annealing above $220^{\circ} \mathrm{C}$ transesterification reactions lead to the formation of a homogeneous phase which in the case of the $\mathrm{PC} /$ polyester blend consists of random copolymers. For the PC/PMMA blends, the details of the homogeneous phase are not yet clearly understood. One possibility is that a random network forms where PC chains provide links between PMMA backbones. At intermediate temperatures near $200^{\circ} \mathrm{C}$, there is a competition between the effects of immiscibility and transesterification. The resulting 
morphology contains domains with broad interfacial regions for both types of blends.

Continuing investigations have focussed on using dielectric spectroscopy as a characterization tool for blends undergoing transesterification. For the PC/polyester case, it is observed that the glass transition temperatures and the dielectric $\epsilon_{0}{ }^{\prime}-\epsilon_{\infty}{ }^{\prime}$ increase dramatically upon annealing at $220^{\circ} \mathrm{C}$. The data strongly suggest that, in the homogeneous, transesterified state, the dipoles of both components relax collectively. By contrast, only minimal changes are observed after annealing the initial non-transesterified film at $160^{\circ} \mathrm{C}$. The latter result, however, implies that some residual mixing is present even in the phase separated films. Dielectric measurements have been performed on a variety of compositions and thermal treatments. Overall, the data are consistent with the results of SANS and DSC.

\section{Effects of Strong Interactions and Transesterification Reaction in Thin Films}

The behavior of polymer blends near surfaces and in thin film geometries is technologically important for applications in areas such as thin film adhesion and structural integrity of coatings. Surface analytical techniques such as $\mathrm{x}$-ray and neutron reflectivity and atomic force microscopy were employed to measure the surfaces and interfaces of thin films of polymer blends with nanometer resolution. Complexation and transesterification mechanisms are investigated as strategies to alter the phase behavior and control miscibility in such systems. These mechanisms may also serve to enhance thin film strength and adhesion.

The kinetics of interfacial mixing were examined in a thin bilayer of lightly sulfonated polystyrene and poly(N,N'-dimethylethylene sebacamide) nylon. Neutron reflectivity was used to monitor the interface structure as a function of annealing time at a temperature above the nylon melting point, but below the glass transition for the sulfonated PS. The interfacial mixing was inhibited above a given sulfonation level due to the strong interactions between styrene sulfonate and the amide group. Intermolecular complexes that act as physical crosslinks form upon mixing the two polymers, and this slows down the diffusion and interfacial mixing processes.

The structure of thin film blends of PC and deuterated PMMA on silicon substrates was studied as a function of annealing time at $200^{\circ} \mathrm{C}$. Initially, there is a rapid enrichment of PMMA at the silicon substrate surface. At longer times, this trend is reversed due to the transesterification reaction which tends to mix the chains near the interface. At later times, atomic force microscopy of the air/polymer interface shows a hole-pattern which evolves into droplets, similar to a roughening phenomena for immiscible blends. The late stage

coalescence is inhibited by the block copolymer at the interface of the droplets as in the case of nonreactive blends stabilized by block copolymers.

Interfacial Characterization and Phase Separation in Thin Film Polymer Blends and Block Copolymers 
A. Karim, B. Factor, J. Douglas and C.C. Han

\section{Objectives:}

The objectives are to investigate the effect of surface interactions on polymer phase separation in thin films of binary polymer blends and the interfacial organization of diblock copolymers and adsorbed polymers. Novel techniques of X-ray and neutron reflectivity along with ellipsometry, and optical and atomic force microscopy are employed to probe these phenomena.

\section{Accomplishments:}

Many applications of polymeric materials involve confinement of blended materials in narrow dimensions. The critical temperature for phase separation in polymer blends films depends on the film thickness and on the nature of the polymer-surface interface. To investigate these phenomena a project was initiated to map out the thin film phase diagram of a classic binary polymer blend, deuterated polystyrene (dPS) and polyvinylmethylether (PVME) Results on 0.1 micron thick films of varying compositions of dPS and PVME establish an important result that the thin film phase boundary of these materials is stabilized as compared to the bulk due to surface interactions and finite size effects.

A novel surface roughening transition is observed in binary polymer blends. In dPS/PVME thin blend films cast on surface modified silicon substrates, the phase separation process is accompanied by a unique pattern evolution at the free air boundary of the film.

A light scattering cell for in-situ study of phase separation in thin films under high vacuum and at elevated temperatures was built and successfully tested.

The phase behavior of polymer blends may also be altered by addition of block copolymers. A key issue in these materials is the localization of the junction points and chain ends. A strong localization of diblock junction points within strongly segregated lamellar block domains is concluded from studies of the interface widths in thin films of diblock copolymers of polystyrene-poly(2vinylpyridine) (PS-P2VP). In contrast, the chain ends have a broad distribution within the domains with a maximum concentration in the middle of the domains.

The distribution of PS and P2VP homopolymer in PS-P2VP diblock copolymer domains was also studied. Both homopolymers were segregated into their respective block domains up to homopolymer volume fractions of $20 \%$, without disrupting the lamellar microstructure of the films.

Polymers at solid-liquid interfaces are used to modify flow properties of fluids, and often find application in colloid stabilization. The interfacial properties of chemically end-grafted polystyrene chains at the silicon/solvent interface are studied by neutron reflection and are compared with theoretical predictions. In addition, the adsorption of a surface active polymer, polydimethylsiloxane, from poor solvents as a function of polymer concentration and temperature was investigated by ellipsometry for the surface layer concentration and 
thickness.

\title{
Shear Mixing/Demixing of Polymer Blends
}

\author{
A.I. Nakatani, D.S. Johnsonbaugh, J.F. Douglas, S. Kim ${ }^{1}$ S. $\mathrm{Li}^{2}, \mathrm{M}$. Dadmun and \\ C.C. Han \\ ${ }^{1}$ University of Wisconsin, Madison, WI \\ ${ }^{2}$ East China University of Chemical Technology, Shanghai, China
}

\section{Objectives:}

The objective is to understand the relationship between processing conditions and polymer blend morphology by using light scattering and optical techniques. These studies require the development and construction of a number of different experimental devices to perform insitu studies of polymers under flow. The investigations on homopolymer blends will establish baseline conditions for studies involving interfacial modification of polymer blends.

\section{Accomplishments:}

Using the previously developed shear light scattering instrument, new data involving a twostep shear quench of a polymer blend solution was obtained. A polystyrene (PS) and polybutadiene (PB) blend (50:50 by weight) dissolved in dioctyl phthalate (DOP) (8\% total polymer by weight) was subjected to a two-step shear quench with a delay time between the two shear rates to examine the phase separation kinetics by light scattering. In these experiments, the sample was allowed to reach steady state at high shear rate, then "shearquenched" to a lower shear rate. A delay time, $t_{d}$, was employed between the two shear rates in order to control the amount of structure development before the second shear rate, $\dot{\gamma}_{2}$, is applied. By controlling the structure evolution before application of $\dot{\gamma}_{2}$, the hypothesis that the existing structure affects the coarsening kinetics after cessation of $\dot{\gamma}_{2}$ was tested.

Three conclusions may be drawn from this study: i) The slow relaxation of anisotropic scattering patterns produced by low shear rates to an isotropic pattern was found to be independent of the sample history prior to the deformation. The nature and extent of preexisting structure in the sample is therefore not responsible for the slow relaxation behavior. ii) The two-step experiments revealed that the coarsening process is accelerated during the second shear rate, then decelerates after cessation of the second shear rate. iii) The coarsening behavior in the directions parallel and normal to flow are coupled. When phase separation occurs during $\dot{\gamma}_{2}$ normal to flow, phase separation parallel to flow only occurs in a q-independent fashion, after cessation of $\dot{\gamma}_{2}$. Spinodal-like phase separation parallel to flow is only observed when the second shear rate suppresses growth in all directions.

These experiments are important in polymer blend processing to understand the effect of preexisting morphology on processing and the redevelopment of morphology after processing. The results showed that pre-existing states do not control phase separation kinetics. Instead, only the processing shear rate controls the final coarsening behavior. 
Accelerated coarsening was observed during second shear rate and decelerated coarsening observed after second shear rate. It appeared that a greater extent of mixing occurs normal to flow direction. This is opposite to results obtained by SANS for undiluted polymer blends, but must be confirmed by SANS.

A new instrument was constructed which combines some of the technology of the shear light scattering instrument described above with a phase contrast microscope. The resulting instrument records both the light scattering images of polymers under shear as well as the microscopic images of the polymers under flow in real space. This is an important advance in our capabilities for studying polymer behavior under shear.

\title{
Structure Change and Alignment of Block Copolymers Under Shear
}

\author{
C.L. Jackson, A.I. Nakatani, F.A. Morrison ${ }^{1}$, K.A. Barnes ${ }^{1}$, J.W. Mays ${ }^{2}$, M. \\ Muthukumar', J.F. Douglas and C.C. Han \\ ${ }^{1}$ Michigan Technical University, Houghton, MI \\ ${ }^{2}$ University of Alabama, Birmingham, AL \\ ${ }^{3}$ University of Massachusetts, Amherst, MA
}

\section{Objectives:}

The objectives are to analyze the morphology of a block copolymer melt near the orderdisorder transition temperature as a function of temperature and shear rate, and to better understand the processing characteristics of these materials.

\section{Accomplishments:}

The effect of shear flow on the morphology and order-disorder transition temperature, $T_{\mathrm{ODT}}$, of a block copolymer of polystyrene- $\mathrm{d}_{8} /$ polybutadiene/polystyrene- $\mathrm{d}_{8}$ (SBS) with a block architecture of $8 \times 10^{3} / 54 \times 10^{3} / 8 \times 10^{3} \mathrm{~g} / \mathrm{mole}\left(\mathrm{M}_{\mathrm{w}} / \mathrm{M}_{\mathrm{n}}=1.05\right)$ was analyzed near the ODT using in-situ SANS, and on sheared and quenched specimens using SANS and TEM. The overall composition of the polymer was $23 \mathrm{wt} . \%$ polystyrene, and the ordered phase of this sample is a hexagonally packed cylindrical array, with unit cell parameter $a=25 \mathrm{~nm}$. The quiescent $T_{\text {ODT }}$ of this material was $116 \pm 5^{\circ} \mathrm{C}$, by dynamic mechanical measurements. The in-situ SANS showed that the cylinders quickly align in a shear field as observed previously. The TEM results on the sheared and quenched specimens indicated that a shear-induced change in the packing symmetry of the microphase separated cylinders occurred at sufficiently high shear rates. The transformation is akin to "martensitic transformations" observed in many different metals under deformation. This result implies that theories developed for atomic scale structural changes in metals may be more broadly applicable to the block copolymer class, on a meso-size scale. The $T_{\text {ODT }}$ under shear by in-situ SANS was measured to shift upward by up to $20^{\circ} \mathrm{C}$, as the shear rate was increased. Studies of this type are important to understand the effect of flow on the extrusion processing of these industrially important thermoplastic elastomer materials. 


\title{
Synthesis and Characterization of Organic-Inorganic Interpenetrating Polymer Networks
}

\author{
B.J. Bauer, Da-Wei Liu, C.L. Jackson, A.I. Nakatani and J.D. Barnes \\ Objectives:
}

The objectives are to synthesize and characterize new materials composed of interpenetrating networks of organic polymers and inorganic polymers of various compositions and understand the relationship between the synthesis and the ultimate morphology of these new hybrid materials. Many commercially important polymeric resins are filled with silica to modify the thermal, electrical, and physical properties of the resin. If the silica can be formed chemically in the presence of the resin, a whole new class of materials will be created that have new morphologies, and therefore new properties, that do not presently exist.

\section{Accomplishments:}

Interpenetrating polymer networks (IPNs) have been synthesized by performing sol-gel chemistry and conventional organic polymerizations in mixtures of the monomers. The organic polymers were acrylate, methacrylates, or epoxies, and the inorganic phase was $\mathrm{SiO}_{2}$ formed by hydrolysis of orthosilicates. Polymerizations were conducted simultaneously or sequentially at a variety of relative rates, and the chemistry was designed to allow different amounts of grafting between the components. The morphology was characterized by transmission electron microscopy and small angle neutron and $\mathrm{x}$-ray scattering. Wide variations in morphology were observed depending on the polymerization conditions, ranging from grossly phase separated to dendritic to finely divided structures (at a $100 \AA$ size scale). The phases ranged from mixtures of the two components to relative pure phases. The interface between the phases ranged from very narrow to relatively broad. The thermal stability of the IPNs was significantly different from samples made by physically mixing silica into polymeric resins due to the continuous silica phase.

\section{Pressure Dependence of the Phase Behavior of Polymer Blends}

\section{K. Migler}

\section{Objective:}

Polymers are typically processed under conditions of high temperature, pressure and shear. Temperature and shear greatly affect the miscibility of polymer blends, but much less is known about the effect of pressure. Since miscibility can determine the ultimate material properties, it is planned to explore the fundamental relationship between pressure and the phase behavior of blends. The pressure dependence of the following important items will be measured: the spinodal and binodal lines, the chi parameter, the radius of gyration, and domain sizes. This information will be essential in the development of improved equation of state theories. 


\section{Accomplishments:}

Construction of an apparatus to carry out small angle neutron scattering studies of polymer blends under high pressure and temperature is almost complete. The pressure generator and sample cell are both operational and are capable of generating and maintaining pressures in excess of $100 \mathrm{MPa}$ (about $15,000 \mathrm{PSI}$ ) and temperatures up to $200^{\circ} \mathrm{C}$, which are typical processing conditions.

\section{Characterization of Polvmer Blends during Processing}

K. Migler and D. Johnsonbaugh

\section{Objective:}

As a natural extension of the previously described project "Shear Mixing/Demixing of Polymer Blends", a project is developed to carry out in-situ characterization of polymer blends in a laboratory scale polymer processor. Microscopy and light scattering techniques will be adapted to characterize the state of the blends, focusing on miscibility, domain size, shape and dynamics of the blend. By development of these in-situ techniques, insights are gained into the role of the important processing parameters such as shear, temperature, pressure and polymer composition.

A small laboratory mixer and twin screw extruder have been acquired for implementation of the optical measurement systems. 


\section{POLYMER COMPOSITES PROGRAM}

\section{Goals}

The polymer composites program seeks to facilitate the introduction of these light-weight, corrosion-resistant materials into commercial applications. By using the high specific strength and stiffness of these materials, it is possible to make products that are significantly improved and therefore more competitive in international markets. Composites can impact industries as diverse as transportation, construction, marine, off-shore oil, medical devices, and sporting goods. The major focus in this program is the automotive industry because that is the market where composites probably have the biggest potential for near term impact. Nevertheless, the work also has direct relevance for the use of composites in off-shore oil platforms, infrastructure, construction and aerospace.

The program plan was developed in conjunction with industry through a series of workshops, exchange visits, and consultations. In these activities, industry identified the two most important barriers to increased use of composites: the need for more rapid, reliable, costeffective fabrication; and the need for a better understanding and predictive capability for long term performance. In response to this challenge, the composites program initiated two tasks: one on processing science and the other on durability.

The long term goal in the task on processing science is to develop technology to monitor, understand, model, and control the events that occur during composite fabrication. The program has an interest in four processing methods: autoclave cure, press molding, tow placement, and liquid composite molding (LCM). The vast majority of the work, however, focuses on LCM since this fabrication method is of great interest to all industry sectors and is the consensus choice of the automotive industry as the method with the most promise for making structural automotive parts. The approach in this task involves three steps. First, measurement tools are developed and utilized to characterize the material properties that control processing, for example, permeability. Second, sophisticated process simulation models are formulated to analysis the effects of processing parameters rapidly and inexpensively so they can be optimized. Finally, process monitoring sensors are developed and utilized to provide feedback for verification and improvement of the simulation models and to help develop the technology for on-line process control. The current activities in this task involve five projects, one of which applies process sensors and controls to a specific problem as part of an industry-university-government program sponsored by the Advanced Research Projects Agency.

The work in durability focuses on degradation resulting from moisture attack on the composites. The long term goals in this task are to identify the chemical and physical mechanisms of degradation, to develop effective test methods, and to formulate reliable predictive models. The program utilizes glass fibers with rapid cure resins like vinyl esters 
and polyurethanes that are the primary candidates for automotive applications. In addition, the work considers epoxy resins for marine and infrastructure applications. The latter work will expand in the coming year to include graphite fibers as well as glass. The usual approach in durability studies is to characterize the behavior of composite samples exposed to various environmental conditions. Because a composite is such a complex system, however, the interpretation of the data is difficult. The work here uses two approaches to address this problem. First, composites are formulated with systematic variations in constituents and processing conditions. A comparison of durability data for the various samples can then provide insight into the possible sources of any differences that occur. Second, durability is measured on the individual components of the composite, i.e., the resin, the fiber, and the fiber-matrix interface. The information obtained can provide another insight into the degradation mechanisms. There are currently three specific projects in this task.

The composites program has active collaborations with a wide variety of industries and universities. The industries include the big three automotive companies and their joint venture called the Automotive Composites Consortium. Other cooperative programs include Dow, Owens Corning, General Electric, Northrop-Grumman, Boeing, Atlantic Research, and a consortium of industries, universities, and government laboratories lead by NorthropGrumman, AlliedSignal, Allison Engine Company. There is also a collaborative effort with the Textile Research Institute.

A number of scientists contributed to the program by working at NIST during the past year. Prof. W. Bradley from Texas A\&M University spent 7 months on sabbatical, and Dr. S. Ranganathan, who has a postdoctoral position at the University of Delaware, is working here in a joint program. Dr. K. Macturk joined the program as a NIST/NRC Postdoctoral Associate, and Mrs. D. Woerdeman is continuing her thesis research in our laboratories as part of program with Johns Hopkins University. Dr. C. Moon of Pusan National Fisheries University in Korea completed his sabbatical at NIST during the past year while other guest scientists who contributed to the program include M. Diallo of Ecole Polytechnique, G. Mychajluk of Stevens Institute of Technology, and S. Kondagunta from West Virginia University.

\section{FY-94 Significant Accomplishments}

- A preliminary model has been developed to predict the processing conditions under which pressure from fluid flow will deform the fiber preform in liquid composite molding. Industrial processes and flow analyses rely on the assumptions of a rigid preform; however, the desire to increase processing speed can produce conditions which violate this assumption. The result can produce poor quality parts. The model is a first step toward identifying such conditions so they can be avoided.

- An evanescent wave optical fiber fluorescence sensor has been developed which can be incorporated into the preforms used to reinforce composite parts. This optical fiber is inexpensive and can be used in a surface-sensitive geometry (evanescent 
wave) which can study the fiber/matrix interfacial region. A fluorescent probe has been obtained that not only functions well in epoxy systems, but also gives identical information in bulk and surface-sensitive sensing modes.

- An evanescent wave IR sensor has been constructed and mounted into the wall of a mold for liquid composite molding. Software for data collection, storage, and manipulation has been developed to calculate the degree of conversion and output this to the process control computer. A control program was developed based on a model assisted feedback control algorithm that adjusts the chemical reaction rate by manipulating the temperature through a base-level controller.

- A simple, semi-analytical model has been developed to predict the effects of tow microstructure on permeability for transverse flow through a regular array of porous elliptical cylinders that represent the reinforcement tows in a preform. The arrays can range from rectangular to proportionally hexagonal. The predictions have been verified by comparisons with rigorous finite element calculations.

- A finite element simulation for predicting the permeability for arbitrarily complex textile micro-structures was developed. Comparisons have been made with experimental results for flow in a unidirectional material, and the results agree to within $20-40 \%$. This is a major advance since this degree of agreement can be useful for many applications and previous models have been in error by more than an order of magnitude.

- An experimental procedure has been demonstrated for estimating the degradation of both the fiber and the fiber-matrix interface when single fiber fragmentation specimens are exposed to moisture. This technique will now permit systematic studies of different fibers, surface treatments, and resins on environmental stability of composite systems.

\section{Liquid Composite Molding: High Speed Flow Behavior and the Development of Permeability Measurement Techniques and Data}

R.S. Parnas, K.M. Flynn, C.R. Schultheisz and D.L. Hunston

\section{Objectives:}

The objectives in this project are (1) to develop the technology for measuring the critical input parameters for process simulation models, (2) to use this technology and establish a data base of values for industry, and (3) to define the limitations of such models. The parameters of interest include the properties of the reinforcement materials that are combined to form the preforms used in LCM. As indicated by the objectives, this work couples directly with the project on the Development and Verification of Process Simulation Models.

\section{Accomplishments:}


The approach is to study the nature of flow through porous medium (i.e. the reinforcing material or preform) and to characterize the resistance to this flow or permeability. The work develops reliable methods to determine permeability, utilizes these methods to characterize a wide range of reinforcement materials, and produces standard reference materials for permeability that industry can use to verify their experimental methods. A number of effects which complicate permeability measurement are investigated. For example, permeability is defined in terms of steady-state flow with the reinforcement fully saturated with resin. When the resin is first injected, however, the reinforcement is not saturated and anomalous effects are sometimes seen for this so-called unsaturated flow. Understanding these effects is critical to the reliable measurement and use of permeability data. Finally, analyses that use permeability have limitations. For example, when the flow rates are high enough, the forces generated by the fluid can deform the reinforcement materials thus changing their permeability. The research in this project seeks to define these situations and to develop simple models to predict when and how they occur.

The differences between saturated and unsaturated flow have been indisputably documented by the NIST permeability measurement laboratory and accepted by the composites processing community. The causes of these differences have been reliably attributed to capillary effects and to the structural heterogeneity of the preform material by both NIST workers and a number of others as well. Furthermore, the unsaturated flow has been linked through models and experiments to void formation. In slow flows, where capillary effects dominate, micro voids can form, and in faster flows, where viscous effects dominate, macroscopic voids may form. Voids, or residual porosity, reduce both the as-manufactured performance of a composite as well as the long term performance, and must therefore be minimized or included in the design criteria.

A statistically designed experiment was conducted with two types of materials, a random mat and a 3-D weave, to generate the data necessary for a standard reference material and to document the differences between saturated and unsaturated flow. The random mat material showed reproducible flow behavior dominated by capillary effects, and the apparent permeability during unsaturated flow was higher than the saturated (or Darcy) permeability. The 3-D woven fabric showed reproducible flow behavior dominated by structural heterogeneity, the apparent permeability during unsaturated flow was initially lower than the Darcy permeability, and became higher than the Darcy permeability later in the experiment as predicted by a previously developed model. These differences between saturated and unsaturated flows must be included in the next-generation processing models to properly design molds and optimize injection strategies to minimize void formation.

The data generated for the random mat and 3-D woven fabric, as well as a number of other reinforcements of engineering interest, are being compiled into a data base for dissemination. An example of the usefulness of such a data base was also illustrated by performing flow experiments with multi-layer preforms containing both the random mat and the 3-D woven fabric. In that case, the permeability of each constituent of the preform was required to predict the flow behavior in the multi-layer preform. Since the number of possible 
combinations of reinforcement fabrics is nearly limitless, a data base of permeability will be valuable in the design of the complex preforms required for large structural parts.

Both the analyses of flow behavior and the measurement of permeability rely on the assumption that the preform is rigid in the mold. Two mechanisms of preform deformation may, however, render the assumption invalid, and this project is studying these mechanisms. Deformations can occur during the construction of the preform or when the preform is placed in the mold. This may significantly alter the permeability in localized regions. Experiments have been initiated with the help of the Textile Research Institute to measure permeability for highly deformed reinforcements. The second reason for preform deformation is the forces exerted on the preform by the resin during high speed injection. A model has been developed to predict the processing conditions under which this may be expected. A major use of this model is to indicate where a standard Darcy analysis would be invalid since preform deformation can open flow channels at the mold walls which are not accounted for by usual flow analysis models.

The preform deformation model couples an equation for the mechanical properties of the preform material with a fluid flow model. However, the combined problem of flow in a deformable porous medium with an adjacent open channel that changes with time is quite formidable. Thus, simplified models have been used in an effort to provide a screening analysis to determine when such deformation may be important rather than to quantitatively solve the entire problem. The single most important result is that preform deformation should not be expected if the injection pressure is held lower than the mold clamping pressure required to compress the preform in the mold to the desired fiber volume fraction. Larger injection pressures can be tolerated in some cases, and the model computes the tolerable operating conditions as a function of the preform permeability and stiffness, the mold clamping pressure, and the mold geometry.

\section{Liquid Composite Molding: Development and Verification of Permeability Prediction $\underline{\text { Models }}$}

F.R. Phelan, S. Ranganathan, ${ }^{1}$ G. Wise, M.L. Diallo ${ }^{2}$ and R.S. Parnas

${ }^{1}$ University of Delaware, Newark, DE

${ }^{2}$ Ecole Polytechnique, Montreal, Quebec, Canada

\section{Objective:}

The objective in this project is to develop theoretical tools for predicting the permeability tensor of the fiber preforms used in Liquid Composite Molding (LCM). As a result, the work interacts directly with studies in the previous project where the permeability is being experimentally measured and in the next project which uses the permeability data as input for the process simulations. Permeability depends on the detailed microstructure of the preform material; the number of potential preform architectures and the range of possible volume fractions are very large. At present, the permeability must be measured in the laboratory, but the experiments are difficult and time consuming. Moreover, for some materials there is 
not an adequate means of getting the preform into permeability measurement molds without significantly altering the material microstructure. Consequently, the development of tools for predicting permeability as a function of microstructure is of great practical importance. It is also be a first step in establishing processing-performance relations since the composite properties also depend on the microstructure.

\section{Accomplishments:}

In the approach to the problem developed here, the flow inside the porous fiber tows is modeled using the Brinkman equation given by

$$
\mu \nabla^{2} v-\eta K^{-1} \cdot v=\nabla P
$$

where $v$ is the velocity, $K$ is the intra-tow permeability, $P$ is the pressure, and $\eta$ and $\mu$ are viscosities. The flow outside the tows is modeled using the Stokes equation. The BrinkmanStokes approach allows intra-tow flow to be taken into account in a manner consistent with the proper tow-fluid interface boundary conditions. Analytical models based on flow in ideal arrays of solid cylinders are used to predict the intra-tow permeability of the textile weaves needed in the Brinkman equation. Thus, this approach to permeability modeling enables predictions from first principles based entirely on the microstructure of the preform without any adjustable parameters.

During the past year, two different models were developed using the approach above. The first model employs a simple semianalytical expression for predicting the transverse permeability of regular arrays of aligned porous cylinders with elliptical cross-sections. This serves as an approximation to the structure of unidirectional preform materials; the geometry is shown in Figure 1. This work is an extension of a model developed last year for predicting the transverse permeability of rectangular arrays of aligned elliptical cylinders. This year, the model has been extended to non-rectangular arrays.

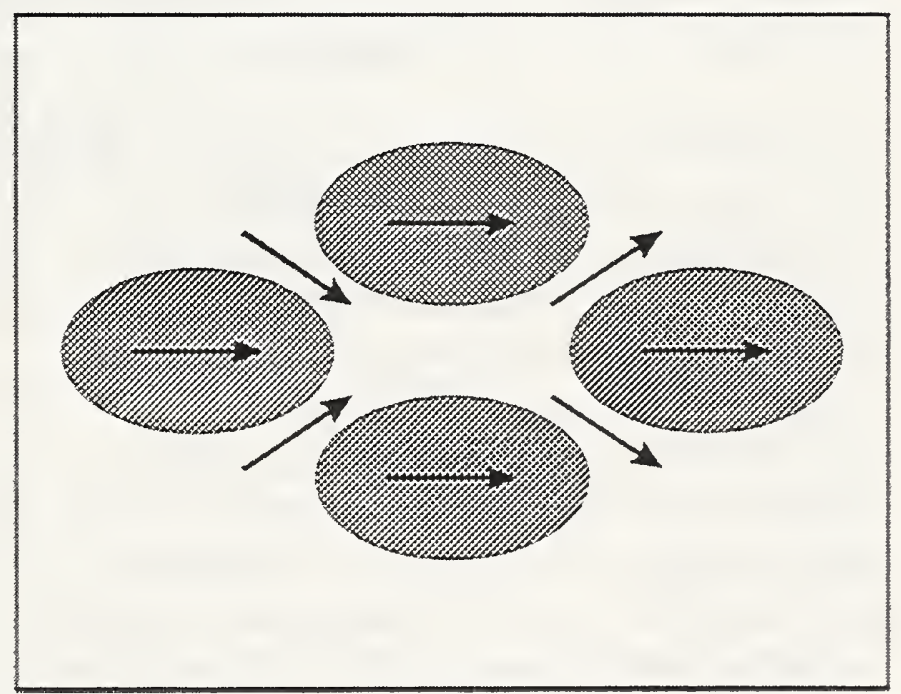

Figure 1: Geometry for transverse flow in a periodic array of porous cylinders.

The starting point for the work was a rigorous finite element analysis of flow in the geometry. The analysis showed that even for very highly permeable cylinders, at close spacing, most of the pressure drop in the system occurs in the narrow gap between adjacent cylinders, in a direction tangent to the line connecting the points of closest approach. This was an important result, and enabled an approximate analysis based on lubrication theory. 
The result is a semi-analytical model for the overall bed permeability given by

$$
K_{\text {cell }}=\frac{L}{H} \cdot \frac{1}{f}
$$

where

$$
f=\int \frac{d x}{K^{1.5}\left[\frac{g^{3}}{6}-(h+2 g+j)-\frac{g^{2}[g \tanh h+2][g \tanh j+2]}{4[g \tanh h \tanh j+\tanh h+\tanh j]}\right]}
$$

and $L$ and $H$ are the overall length and height of the unit cell, $g$ is the gap spacing between tows, $h$ and $j$ are the distances from the tow fluid interface to the centerline of the tow (note that $g, h$, and $j$ are functions of $x$, the distance along the tow in the flow direction).

Note that the expression above is a function only of the tow shape, packing characteristics, and intra-tow properties. The integration is easily performed numerically, and is far less work than carrying out an entire finite element analysis. The model predictions have been verified by comparing with rigorous finite element calculations using the commercial software package FIDAP. Good agreement was obtained for a range of volume fractions, tow shapes and tow configurations.

A comparison plot is shown in Figure 2 which gives the permeability for a model material with porous tows, $\mathrm{K}_{\text {porous }}$, normalized by the permeability for the same material with solid tows, $\mathrm{K}_{\text {solid. }}$. The model shows the influence of intra-tow permeability on the overall bed permeability. The latter increases as the inter-tow packing and the degree of tow ellipticity are increased. The work also shows that the shape, intra-tow permeability and spatial arrangement of the individual tows all significantly influence the overall permeability of the medium. Hence, an accurate representation of the microstructure is essential for accurate permeability prediction. The intra-tow influence is particularly critical for "stacked" geometries where previous models predict a zero permeability. The only requirement necessary to use the model

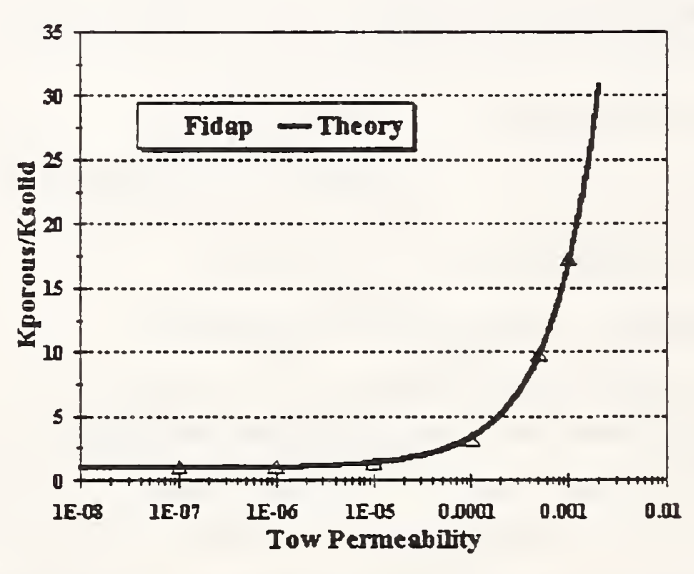

Figure 2: A comparison of the semianalytical model and numerically calculated values of permeability as a function of tow porosity at a nominal volume fraction of 0.8 . 
is that the array being modeled be periodic such that there is a unit cell representative of the entire medium. While the development and testing was limited to the case of tows with elliptical cross section, the method is general and can easily be extended to inclusion nonelliptical cross sections. Therefore, the transverse permeability of most fibrous unidirectional media may be estimated using the approach.

A second type of model for permeability prediction involves the development of a finite element simulation for predicting flow in 3-D fibrous porous media. This simulation employs the Brinkman-Stokes approach outlined above for modeling the flow, but the use of a finite element scheme makes it possible to obtain solutions for arbitrarily complex geometries provided a mesh of the microstructure can be generated.

To test this model, numerically calculated predictions for permeability have been compared with experimentally measured values for flow in Knytex D155 unidirectional material in a nested stacking configuration. This material and configuration were chosen to achieve a controlled preform microstructure. Two sets of experiments were performed, the first with the material in the as-received state, the second with the material having been washed in an acetone bath to remove the intra-tow binder. For a first effort, the results that have been obtained are encouraging. In all cases, the numerical values are of the same order of magnitude as the experimental values. For the case in which the material was washed in an acetone bath to remove intra-tow binder, the quantitative agreement is within $20-40 \%$. Results for the transverse permeability are shown in the table below:

\begin{tabular}{|c|c|c|}
\hline \multicolumn{3}{|c|}{$\begin{array}{c}\text { Table 1 -- Transverse Permeability }\left(\mathrm{cm}^{2}\right), \text { Knytex } \\
\text { D155, Nested Configuration, Without Binder }\end{array}$} \\
\hline \hline Exp. 1 & Exp. 2 & FEM Model \\
\hline $1.9 \times 10^{-8}$ & $2.1 \times 10^{-8}$ & $1.5 \times 10^{-8}$ \\
\hline
\end{tabular}

In most cases, the numerical predictions are lower than the values measured experimentally. It is believed that this is due to variations in the tow size dimensions. Image analysis reveals that in this material, there is a large distribution of tow sizes with a variation on the order of $\pm 10-20 \%$ of the nominal sizes. Larger gaps in the tow size spacing allow more flow to pass through, hence increasing the permeability. To obtain better quantitative agreement, future work will need to account for statistical variations in microstructure by doing a number of simulations, and weighing the results about the mean. Nevertheless, for many cases, permeability estimates within this degree of reliability can be very useful.

Liquid Composite Molding: Development and Verification of Process Simulation Models

F.R. Phelan and D.L. Hunston 


\section{Objective:}

The objective in this project is to develop and apply models that can simulate the events which occur during the LCM process. To be used effectively, the processing parameters must be optimized for the particular part design and materials being used. Historically this has been done with time-consuming and expensive trial and error methods on full scale equipment. Simulation models can greatly reduce the cost and increase the speed of this task. A wide variety of temperatures, pressures, injection ports, vents, etc. can be inexpensively examined in a mater of hours using simulations. In addition, trade-offs such as the interaction between injection speeds and pressures can be considered. As a result, processing conditions can be chosen to provide a highly efficient fabrication process.

\section{Accomplishments:}

In previous work, a simulation for the mold filling phase of LCM was developed. The simulation uses a Flow Analysis Network (FAN) technique to predict and track the movement of the free-surface, and a finite element method (FEM) to solve the governing equation set for each successive flow front location. The flow model used in the simulation is based on Darcy's law given by

$$
\boldsymbol{v}=-\frac{\boldsymbol{K} \cdot \nabla P}{\eta}
$$

where $v$ is the velocity, $K$ is the permeability tensor, $\eta$ is the viscosity, and $P$ is the pressure. A variety of element types have been incorporated into the simulation enabling modeling of flow for a variety of conditions of industrial interest including runner distribution systems, 2.5-D shell geometries, and fully 3-D flows.

Work during the past year focused on two areas: the initial steps in the development of a simulation model for Injection/Compression Composite Molding (ICCM), and the application of the existing LCM model to industrial problems in cooperation with a variety of companies.

Work in the first area came about because the ICCM process has been selected by the automotive companies as the most promising method for fabrication of large structural parts. The process is begun as in regular LCM with the placement of a preform into the mold. However, the tool is left partially open so that the preform is not fully compressed, and an initial charge of liquid is injected into the mold, partially filling it. The tool is then fully closed, forcing the fluid charge to fill the entire volume of the mold, and compressing the preform to its final net shape. There are two main variants of the process. In the first, the upper mold wall partially compresses the preform, and there is no gap in between the preform and the mold wall for fluid flow. In the second, the upper mold wall does not come into contact with the preform prior to injection, which leaves a gap for fluid flow in between the preform and the mold wall.

To examine this problem, an analysis of ICCM for thin shell parts containing multi-layer 
preforms with no gap has been completed. The model takes into account the changes in the thickness of the individual layers in a preform stack to calculate new volume fractions and new permeabilities as a function of volume fraction. These new permeability values are then used in the calculation of the next stage of the flow front advancement. In addition, implementation of non-isothermal model for the filling simulation was begun.

In the second aspect of this project, the mold filling behavior of a variety of real parts has been analyzed in cooperation with industry. For the Automotive Composites Consortium, a series of simulations was performed on a cross-member to help design the processing studies they are planning for the coming year. Two other examples of mold filling analyses are a complex automotive tray geometry designed by General Motors and a helicopter rotor shank of interest to Atlantic Research Corporation. Cooperative work has also continued with Northrop-Grumman, and Boeing. These collaborations demonstrate to industry the advantages of simulations in optimizing the fabrication of such structures.

\section{Liquid Composite Molding: Development of Fiber Optic Sensors for Process Monitoring}

D.L. Hunston, R.S. Parnas, J.P. Dunkers, D.L. Woerdeman ${ }^{1}$, B.M. Fanconi, R.E. Lowry and D.G. Blackburn ${ }^{2}$

${ }^{1}$ The Johns Hopkins University, Baltimore, MD

${ }^{2}$ Ceramics Division, NIST

\section{Objective:}

The objective in this project is to develop process monitoring sensors based on spectroscopic measurement methods. This couples strongly with another project, Process Monitoring and Control of Pre-Ceramic Polymers, that is implementing such sensors for process monitoring and control in collaboration with industry. Ultimately, it should also assist the project, Development and Verification of Process Simulation Models, since the sensors can provide feedback for model verification and refinement.

\section{Accomplishments:}

The manufacture of polymer composites presents a special problem when compared to metals because of increased waste and the associated costs due to variability of part quality. The inconsistency in part quality can result from a number of factors including the shelf life of monomer, catalyst poisoning, temperature variations, formulation changes, and operator variations. These factors primarily affect the conversion of monomer to polymer (degree of cure) during processing and thus in-situ monitoring of degree of cure may provide on-line quality control for polymer composite processing with potential to drive down part cost by minimizing part to part variation. Moreover, the incorporation of sensors into composite processing can provide real time data on the flow of the monomer and the state of cure of the polymer for process control.

The choice of sensing technique is highly dependent upon the materials and process involved and the information that is needed. In addition, the technique must be adaptable to the 
manufacturing environment. To satisfy the last mentioned requirement, the work in this project focuses on spectroscopies that can utilize fiber optics to transmit the radiation between the measurement apparatus and the sensor itself. A fiber can also be used as the sensor. This offers the potential for both localized and through thickness measurements because the sensors can be incorporated into the reinforcement. Finally, this approach can be used to monitor various phenomena including fluorescence, Raman scattering, and ultraviolet, visible, near- and mid-infrared absorption, and refractive index changes. Of these various possibilities, attenuated total reflection (ATR) Fourier transform infrared (FT-IR) and fluorescence spectroscopies are currently under investigation.

Infrared spectroscopy was chosen because of the advantages it offers over other techniques and because of the potential it has for overcoming its current limitations. FT-IR spectroscopy is a robust and routine technique that has been studied for over 20 years and gives direct information about the number and type of chemical species present. This is in contrast to other techniques that measure chemical conversion only indirectly. FT-IR can monitor not only the amount of reaction, but also how the molecules are reacting and whether any undesirable side reactions are occurring, down to relatively low concentrations. Also, FT-IR spectroscopy offers the potential to monitor fast reactions since it has the capability to take as many as 10 spectra in a second. Some of the challenges that limit the widespread application of this method are the relatively high attenuation, large diameter, and high cost of infrared transmitting fiber optics compared to silica based fibers.

Two approaches are currently being pursued for implementation of attenuated total internal reflection (ATR) FT-IR spectroscopy. The first involves a surface mounted germanium internal reflection prism sensor for monitoring production of thin parts. The second approach relies on evanescent wave absorption of infrared radiation transmitted through chalogenide or silver halide fibers which can be embedded in thick parts. Most of the work with the prism sensor is conducted as part of the project investigating pre-ceramic polymers while the research here centers on through-thickness fiber-optic methods.

The second spectroscopy being studied involves fluorescence techniques based on either distal or evanescent wave approaches. Previous work using conventional fluorimetry identified two types of probe molecules whose fluorescence characteristics reveal information about the degree of cure. One type relies on the sensitivity of the fluorescence intensity to the micro viscosity while the other hinges on frequency shifts of the fluorescence with changes in polarity. The intensity-type probe is limited by the requirement for an internal intensity standard since the signal may reflect changes in the measurement system as well as changes in the fluorescence intensity itself. For this reason, the current work has focussed on frequency-dependent probes that do not require a standard.

A major accomplishment during the past year was the introduction of a high refractive index glass fiber that allowed surface sensitive evanescent wave sensing geometries to be exploited inexpensively for the first time. A fluorescence system has been constructed and optimized for cure monitoring epoxy composites in an RTM apparatus. Additionally, the evanescent 
wave geometry offers the possibilities of measuring changes very close to the fiber surface, especially if fluorescent probes are grafted to the fiber surface, and of incorporating several sensing elements into a single optical fiber. The latter is of particular significance to industry because of the attendant reduction in equipment and instrumentation that may be realized.

\section{Liquid Composite Molding: Process Monitoring and Control for the Manufacture of Pre-Ceramic Polymers}

R.S. Parnas, J.P. Dunkers, D.L. Hunston, B.M. Fanconi, F.W. Wang, R.E. Lowry and G. Mychajluk ${ }^{1}$

${ }^{1}$ Stevens Institute of Technology, Hoboken, NJ

\section{Objective:}

The objective in this project is to develop a monitoring and control scheme for the initial step of a process that produces ceramic matrix composites from polymeric precursors. The initial step involves making the polymer precursors by LCM, and it is hoped that the use of this process will significantly lower the cost of the final part. This project is one task in a large external program involving industry, government, and academia sponsored by the Advanced Research Projects Agency (ARPA). The program is lead by Northrop-Grumman, AlliedSignal, and Allison Engine Company, but also involves a number of other participants. The goal is to reduce manufacturing costs for aircraft engine components made from ceramic matrix composite; the initial demonstration part is a turbine seal. The work at NIST involves studies using both a model material system and a commercial resin known as Blackglas ${ }^{\circ}$ which will be used to fabricate the demonstration part.

\section{Accomplishments:}

The work with Blackglas is identifying an appropriate sensor for process monitoring, generating a database of cure information on this material, imbedding this sensor in a simple mold, developing a control algorithm, implementing the control software on a laboratory LCM system, and testing the full package. When complete, this technology will be transferred to Northrop-Grumman for implementation in their facilities.

Two types of sensors, based on fluorescence and infrared spectroscopy, were evaluated for application to the Blackglas system. The infrared approach was selected for implementation because it showed the most promise. An infrared data base of cure characteristics has been developed using conventional transmission sampling. Conditions under which quantitative measurements could be made using attenuated total reflection spectra were established since this sampling method will be used for in-situ measurements.

The fluorescence approach, although not implemented, may be useful in other applications of cure monitoring. In this work, a model system was used to follow the hydrosilation reaction of a vinyl-terminated polydimethylsiloxane polymer (vinyl silicone) with a dimethylsiloxanemethylhydrosiloxane copolymer (hydrosilicone). A trace amount of vinyl anthracene (VA) or 
9,10-bis-(phenylethynyl)anthracene (BPEA) is added as a fluorescent probe to a resin consisting of the vinyl silicone, the hydrosilicone, and a catalyst (platinum divinyl complex). These probes were chosen because hydrosilation of the vinyl group in VA or the ethynyl groups in BPEA led to marked changes in the fluorescence properties of VA or BPEA. Furthermore, if the hydrosilation rate of the vinyl group in VA or the ethynyl groups in BPEA is comparable to the hydrosilation rate of the vinyl groups in the vinyl silicone, the changes in fluorescence properties will be suitable indicators of the hydrosilation or the network formation from the vinyl silicone and the hydrosilicone.

In the infrared spectroscopy method that was chosen for implementation, the in-situ measurements will be obtained by mounting an ATR prism into the wall of the LCM mold. The IR light exits the spectrometer and enters the $45^{\circ}$ germanium prism at a near normal angle of incidence where it undergoes total internal reflection and exits the prism. Upon undergoing total internal reflection, a standing or evanescent wave is created on the external surface of the prism which can interact with the Blackglas, ${ }^{\circ}$ and this changes the characteristics of the beam exiting the prism. The light then enters the detector which is mounted remotely via a fiber-optic cable. The data collection, storage, and manipulation have been automated, and the degree of conversion is calculated and sent to the process control computer.

The process control computer is part of a mobile LCM apparatus constructed to test the sensors and control system. The portable LCM facility was built to demonstrate the versatility of the LCM process as well as to permit the interfacing of the LCM equipment to various sensor systems. A process control program has been implemented, and it includes a model assisted feedback control algorithm to optimize the chemical reaction rate. Since the chemical reaction rate cannot be directly manipulated, the cure controller is cascaded to the base-level temperature controller. The algorithm is quite general, can be linked to any cure sensor that provides information on degree of cure, and can be coupled with any kinetics model that is of the form $A=f(C, t, T)$, where $A$ is the monomer concentration, $t$ is the time, $\mathrm{T}$ is the temperature, and $\mathrm{C}$ is a vector composed of the model parameters. The effectiveness of the control algorithm is illustrated with a simulation shown in Figure 3. The expected cure path for a given set of conditions is illustrated by the curve marked "cure setpoint." After altering the activation energy for one of the reactions, however, the simulation predicts that the cure path would follow the curve marked "no control." This is a problem if the desired behavior is the "cure setpoint" curve. By using the control algorithm to adjust the mold temperature, the reaction kinetics can be accelerated to provide a part with a cure path that closely follows the desired trajectory.

\section{Environmental Durability Studies: Development and Use of the Single Fiber} Fragmentation Test to Investigate the Attack of Moisture on the Fiber and Interfacial Strength of E-Glass/Epoxy Samples

C.L. Schutte, M. McAuliffe, C.R. Schultheisz and K.S. Macturk 


\section{Objective:}

This project employs the single-fiber fragmentation test and has two objectives. The first is to develop an experimental protocol to monitor the degradation of strength for both the fiber itself and the fiber-matrix interface as a function of exposure to water. The second is to demonstrate this analysis method by characterizing the durability of the fiber strength and the interfacial strength for an E-Glass/Epoxy system.

\section{Accomplishments:}

This project has succeeded in developing an experimental protocol for studying the changes in relative strength for the fiber and the fiber-matrix interface in a single fiber fragmentation sample exposed to moisture.

The test involves monitoring the fragment lengths as the dogbone is stretched after various exposure times. Some results for this analysis are shown in Figures 4 and 5 for an E-glass fiber with a commercial, epoxy-compatible coating in a dogbone of epoxy exposed to water at $75^{\circ} \mathrm{C}$ for various lengths of time. It is clear from these figures that both components degrade.

To meet the long-term goals of the durability task, a series of follow-on projects will evaluate the relative durability of both the fiber and its interface with the resin as a function of the type of fiber and type of the surface treatment applied to the fiber. This work also couples with a second project in this task that is evaluating how this type of micromechanical data relates to the relative durability of macroscopic composites.

\section{Environmental Durability Studies: Comparison of Test Results for Laminated} Composite Samples and Single-Fiber Composite Specimens

C.L. Schutte, W.G. McDonough, C.R. Schultheisz, W.L. Bradley ${ }^{1}$, S. Kondagunta ${ }^{2}$, C.K. Moon ${ }^{3}$, K.S. Macturk, and D.L. Hunston

${ }^{1}$ Texas A\&M Univ., College Station, TX

${ }^{2}$ West Virginia Univ., Morgantown, WV

${ }^{3}$ Pusan National Fisheries Univ., Pusan, South Korea

\section{Objective:}

The objective in this project is to compare micro-mechanical information obtained using the single-fiber fragmentation test to macroscopic results from experiments on full composites as a function of exposure to water. 


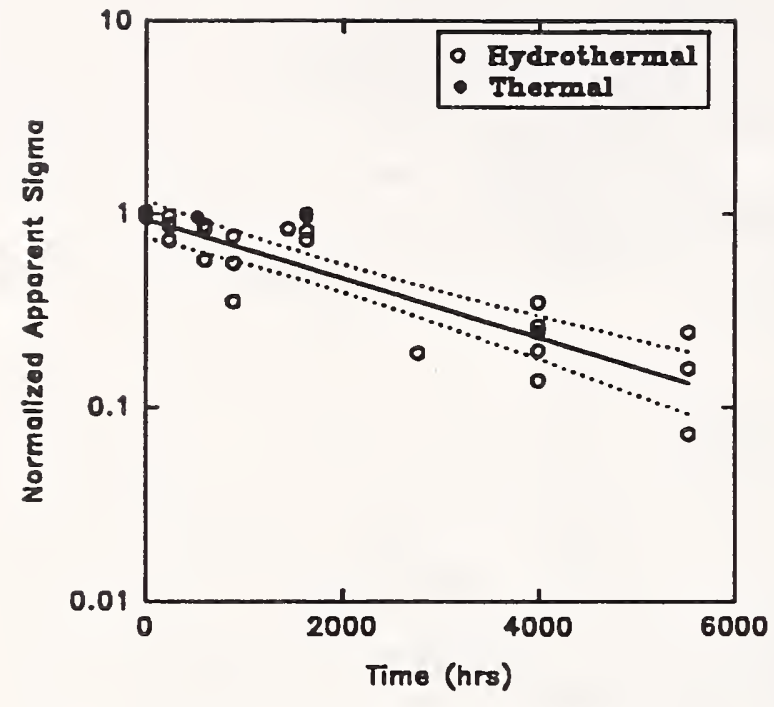

Figure 4: Calculated Strength of the Eglass Fiber, Sigma, as a Function of Exposure Time in $75^{\circ} \mathrm{C}$ Distilled Water.

\section{Accomplishments:}

Understanding of fundamental mechanisms is essential for the accurate predictions of composite lifetime, and the micro-mechanical tests offer results for a simplified geometry that can help elucidate mechanistic information. The studies in this project attempt to bridge micro-mechanical data with macroscopic performance for samples that are exposed to $25^{\circ} \mathrm{C}$ and $75^{\circ} \mathrm{C}$ distilled water for varying lengths of time. The work utilizes an EGlass/Epoxy model system because the initial focus in the overall task is glass fiber composites due to their lower raw-material cost and larger volume market potential as structural composites compared to those for carbon fiber composites. Furthermore, enabling industry to define the limits of durability for glass fiber composites compared to more expensive carbon fiber composites would assist in their risk analyses when deciding which material to chose for a given application and a particular required

performance.

Thus far, the following tasks have been performed in this project: a plan was developed for sample preparation and testing, the single-fiber samples were fabricated, fragmentation tests were performed on samples that were dry and partially saturated with moisture, and a procedure was developed for the fabrication of composite test specimens from resin and specially coated fibers. Testing of the resin itself was also completed. Future milestones include the preparation of the composites samples by the end of 1994 and completion of the tests on both the single-fiber and macroscopic composites. The data will then be evaluated and mechanical models will be developed as necessary to correlate the micro-mechanical results from the single-fiber samples with those from macroscopic composites. Finally, the implications of the results for the prediction of long-term mechanical performance will be considered.

The results to date focus primarily on the single fiber fragmentation test. In this test, a dogbone containing a single fiber is loaded in tension after various exposure times to water. The fiber fragments as the load is increased until a point is reached where further loading does not cause additional fiber breaks. This is called mechanical saturation. The fragmentation process is monitored and from these results, the fiber and interface strengths can be estimated.

The initial results suggest that the strengths of the E-glass fiber at lengths that correspond to 
the fragments at mechanical saturation show a fairly constant rate of decrease with immersion time in water. The influence of the water temperature is evident in the changing Weibull parameters that are determined in the calculation, but the strengths appear to be independent of temperature (Figure 6). Dry samples made with an the epoxy-compatible coating on the fibers clearly had stronger interfaces than samples made with fibers having a vinylester-compatible coating.

The samples immersed at $25^{\circ} \mathrm{C}$ for 3000 hours failed from matrix cracks initiated by fiber fractures; this result appears to indicate that the interface is still relatively strong while the matrix fracture toughness is decreasing. Such strong interfaces might actually be a problem in samples with cracks across the fibers if it suppresses the toughening mechanism of crack blunting through debonding along fibers.

The scatter in the data from both the strength of the fibers and the average fragment length at mechanical saturation makes evaluation difficult, but the interfacial shear strengths seem to display a slower rate of decrease than the strengths of the fibers (Figures 6 and 7). The interfacial strengths of the samples immersed at $25^{\circ} \mathrm{C}$ seem to remain approximately constant out to 2000 hours; both coatings apparently result in similar interfacial shear strengths of approximately 35 to $40 \mathrm{MPa}$. Interestingly, at short times, the epoxy-compatible samples immersed at $75^{\circ} \mathrm{C}$ seem to show a higher interfacial strength than the samples at room temperature; this trend may indicate that the higher temperature leads to additional chemical bonding at the interface that outweighs the negative effects of the absorbed water.

In contrast to the data for epoxy coated fibers, the samples with vinylester-compatible

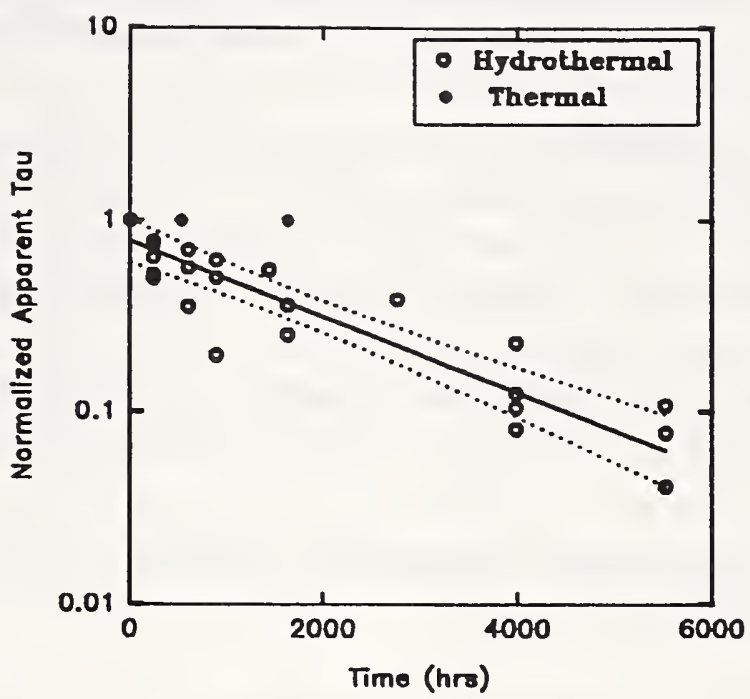

Figure 5: Calculated Interfacial Shear Strength, Tau, for E-glass Fiber/Epoxy Resin as a Function of Exposure Time in $75^{\circ} \mathrm{C}$ Distilled Water.

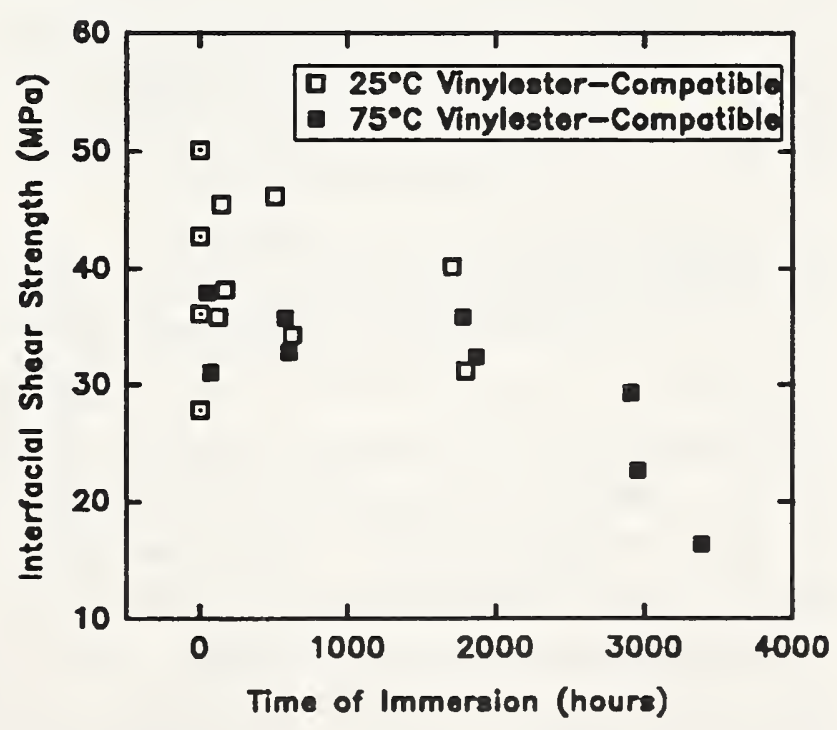

Figure 8: Interfacial shear strength for the vinylester-compatible coating, samples tested dry and after immersion in water at $3425^{\circ} \mathrm{C}$ and $75^{\circ} \mathrm{C}$. 
coatings seemed weaker at $75^{\circ} \mathrm{C}$ than at room temperature, initially (Figure 8). However, the rate of interfacial degradation appears to be increasing for both the epoxy-compatible and the vinylester-compatible coatings at 3000 hours of immersion at $75^{\circ} \mathrm{C}$.

In a composite structure, it is expected that the higher content of fibers will restrict the amount of strain that is caused by swelling in the direction parallel to the fibers. However, there will be much less of a constraint in the directions perpendicular to the fibers, so that the effect of swelling strains on the radial stresses at the interface may be more important in structural applications than the swelling-induced fragmentation and degradation of the strength of the fibers. Finally, the considerable scatter in the data make it clear that further testing is necessary to determine the repeatability and reliability of the results.

\section{Environmental Durability Studies: Development of Processing Methods to Fabricate Urethane Samples}

W.G. McDonough, C.K. Moon ${ }^{1}$, J.P. Dunkers, C.R. Schultheisz, C.L. Schutte and D.L. Hunston

${ }^{1}$ Pusan National Fisheries University, Pusan, South Korea

\section{Objective:}

At the request of the Automotive Composites Consortium (ACC), the Polymer Composites Group is attempting to incorporate an automotive resin into its ongoing effort on durability of composite materials. To this end, a joint effort between the ACC and NIST was established. The long term goal in this effort will be to understand the response of automotive composites to moisture exposure. The first step is a project to develop a processing method that will allow the fabrication of laboratory test specimens. Some tests in the durability effort employ specimens that can be made by the processing method used in the automotive industry to fabricate real parts, i.e., structural reaction injection molding (SRIM). Unfortunately, for one test that is used extensively in the durability program, the single fiber fragmentation test, samples cannot be prepared by SRIM. The motivation for using this test is that it enables the researcher to examine the effects of moisture on the fibermatrix interface in a simple specimen geometry.

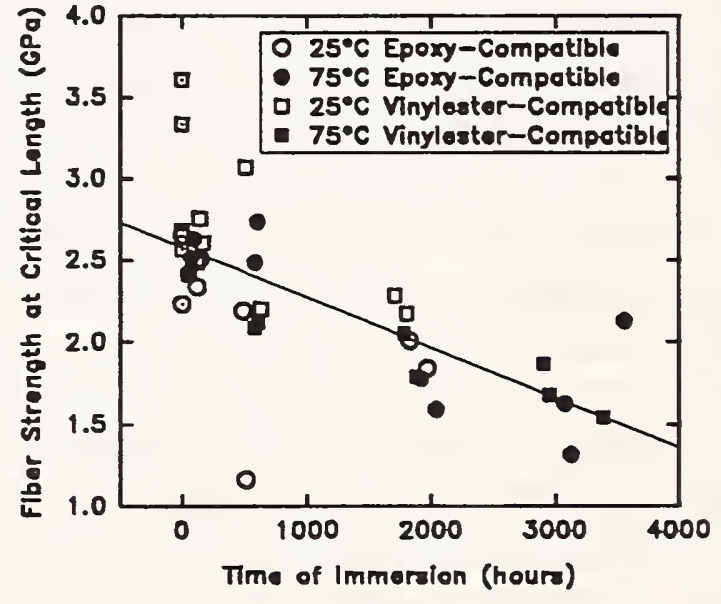

Figure 6: Effect of water immersion on the strength of E-glass fibers at lengths corresponding to mechanical saturation. Epoxy- or vinylester-compatible fiber coatings.

The samples for this test cannot be made 


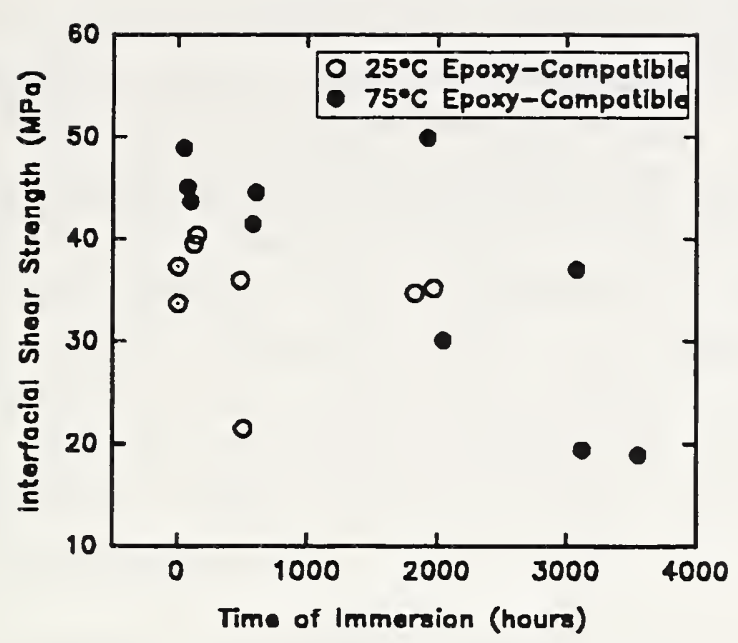

Figure 7: Interfacial shear strength for the epoxy-compatible coating. Samples tested dry and after immersion in water at $25^{\circ} \mathrm{C}$ and $75^{\circ} \mathrm{C}$.

Fragmentation samples are usually made by placing single fibers into silicone mold cavities, filling the cavities with resin, and curing the materials at elevated temperatures and atmospheric pressure. Unfortunately, the resin used in this project, polyisocyanurate, foams at atmospheric pressure. To surmount this problem, a variety of different processing approaches were examined. It was found that by changing the catalyst level in the resin and using the heat and pressure generated in an autoclave, void-free laboratory specimens could be manufactured. In addition, tests showed that these specimens could be successfully examined in single fiber fragmentation experiments; that is, the samples cured in the autoclave had the necessary extensibility to allow the fiber to reach mechanical saturation. For the fragmentation test, mechanical saturation is defined as the point where the fragmentation process ceases. To address the third step, measurements were performed to determine if the laboratory specimens were similar to samples made by the automotive industry using SRIM. The initial results using Fourier Transform Infrared Spectroscopy and Dynamic Mechanical Analysis techniques were very encouraging. The glass transition temperatures for resins made in the autoclave and via SRIM are within $5^{\circ} \mathrm{C}$ of each other (average value $180^{\circ} \mathrm{C}$ ). The FTIR spectra of resins made via the two processing routes are with automotive resins using SRIM due to the violent nature of this processing method which will break the fiber. Consequently, an alternate method must be developed to achieve this goal. This is being done in three steps. First, various processing methods are explored to see if void free laboratory specimens can be obtained. Second, the samples are measured to see if they have sufficient extensibility to be used in the single fiber fragmentation test. Finally, the laboratory samples are compared to materials fabricated by SRIM to ensure that the two materials are similar.

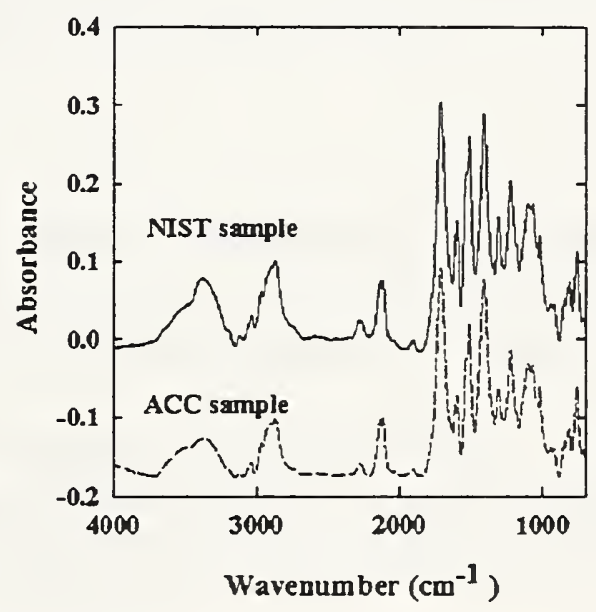

Figure 9: FTIR spectra of polyisocyanate samples made by NIST and the ACC. 
also similar as shown in Figure 9.

To complete the final phase of this project, the testing of laboratory and SRIM samples will be continued, and the results will be used to modify the autoclave process to maximize the similarity with automotive and laboratory materials. This should be completed late in 1995. At that point, a new project will be initiated to include the polyisocyanurate materials in our durability effort. 


\section{VISCOELASTICITY OF SOLID POLYMERS PROGRAM}

\section{$\underline{\text { Goals }}$}

The viscoelastic response of polymers is central to their lifetime, durability, and dimensional stability and, therefore is a determining factor in the performance of these materials in diverse applications from automotive and infrastructure to electronic packaging and imaging applications. The goals of the Viscoelasticity of Solid Polymers Program are to perform research into the mechanics of polymeric resins and to develop models of behavior that form the basis of the prediction of long term stability and performance. The Program is designed around two major themes that have 5-8 year time frames in which to make major accomplishments:

1. Develop the constitutive framework within which to model residual stresses, dimensional stability, and performance of engineering plastics. The program is focusing currently on developments in 'Solid Rheology' for the sub-yield behavior of solid-like polymers based on 'material clock' constitutive models. These show considerable promise for material description and for reasonably easy incorporation into finite element codes.

\section{$\underline{5 \text { year time frame }}$}

a) Evaluate material clock models for nonlinear viscoelasticity and aging responses of amorphous polymers.

b) Develop efficient methods to determine material parameters for the nonlinear constitutive equations.

c) Develop models for the viscoelastic response of 2-phase systems.

d) Do finite element modeling incorporating material clock model constitutive equations for nonuniform thermal histories typical of processing of two material components and injection molded parts.

e) Extend the 'clock' models to moisture diffusion and plasticization effects on polymer material performance.

f) Build finite element model of moisture effects in filled systems.

\section{8 year time frame}

a) Evaluate material clock (or other models) models for nonlinear viscoelasticity and aging responses of semi-crystalline materials.

b) Develop viscoelastic models of yield behavior of amorphous polymers.

c) Develop failure models for the prediction of lifetime of amorphous polymers in stress rupture and fatigue conditions, including time dependence of damage accumulation, crack initiation and propagation.

d) Extend Viscoelastic modeling of yield behavior to semicrystalline polymers. 
2. Establish the scientific underpinnings for the choice of models (constitutive, damage, etc.) required for the above.

\section{$\underline{8 \text { year time frame }}$}

a) In the framework of the physics of glasses determine the basis and limitations of 'clock' models for structural recovery for amorphous polymers.

b) In the framework of the physics of glasses determine the basis for clock models for mechanical response of amorphous polymers.

c) Study the physics of two-phase systems to establish mixing rules for viscoelastic responses.

d) Establish the physical basis for constitutive laws (clock, composite, or other) relevant to the viscoelastic response of semicrystalline polymers.

e) Study the physics of failure using time dependent damage accumulation models and crack incubation, growth and propagation models.

The Program is actively involved with industrial collaborators and designed to accept outside input of information to enhance the model development and transfer to the industrial technology community. Furthermore, the work that addresses the scientific underpinnings determining the choice of constitutive or damage laws interfaces with activities in the Theory and Modeling of Polymers Program.

\section{FY-94 Significant Accomplishments}

- A major survey article 'On the Physics Required for Prediction of Long Term Performance of Polymers and Their Composites' was published in the Journal of Research of NIST.

- $\quad$ Research on 'Physical Aging of Nylon 66' under a CRADA with DuPont and the University of Delaware showed that, unlike amorphous polymers, aging in the semicrystalline nylon occurs above the conventional glass transition.

- In collaboration with the GM/GE Thermoplastic Engineering Design Program, the time-aging time and time-temperature superposition behavior of polycarbonate was characterized from $103{ }^{\circ} \mathrm{C}$ to $145^{\circ} \mathrm{C}$.

- The torque and normal force responses of polycarbonate in torsion were characterized and multi-step strain histories compared with predictions from a strain energy density 'material clock', BKZ, and modified Boltzman constitutive equations.

- The three step stress relaxation response in a non-linear viscoelastic material during physical aging was successfully described using an integral constitutive equation with a strain dependent aging clock. 
- Torsional Dilatometry experiments showed that the coupling between aging and mechanical responses in polymer glasses occurs only in that the mechanical response varies with the structure of the glass. On the other hand, the glassy structure is independent of the applied stress field.

- $\quad$ Preliminary experiments showed how relative humidity-jump experiments are similar to temperature-jump experiments in epoxy glasses.

- $\quad$ Finite element analysis of an inner layer in a printed wiring board showed the impact of polymer viscoelasticity on the evolution of board dimensions over time and how coefficient of thermal expansion measurements can depend on measurement rate.

\section{Physical Aging of Polymers}

G.B. McKenna, C.R. Schultheisz, J.M. Niemiec, G. Gusler, P.H. Verdier, J.D. Barnes, C.L. Schutte, I. Spinu ${ }^{1}$, J. Beckmann ${ }^{2}$ and A. Perez-Castorena ${ }^{3}$

${ }^{1}$ University of Delaware, Newark, DE

${ }^{2}$ Institut für Organische Chemie Universität Potsdam, Potsdam, Germany

${ }^{3}$ Centro Nacional de Metrologia, Mexico City, Mexico

\section{Objectives:}

When a glass forming liquid, such as a polymer melt or rubber, is cooled there is some temperature at which the molecular mobility decreases enough that the material's thermodynamic state or structure cannot attain equilibrium in the time scale of the experiment (determined by the cooling rate). Below this temperature, generally referred to as the glass transition temperature $T_{g}$, glassy materials are unstable with the result that their properties continuously evolve towards a temporally distant equilibrium. Efficient design with and use of polymeric materials require an understanding of the physics underlying the structural recovery and the impact of the changing thermodynamic state on the mechanical properties of the material. A deep understanding of the interrelationships between the physics of glasses and how it influences material performance is essential to successful modeling of material response using nonlinear constitutive equations. Additionally, characterization of the aging response of polymeric materials forms the basis for an advanced materials data base relevant to the performance of polymers in engineering applications where a knowledge of how the dimensions and properties evolve over time is important to good design. The goals and objectives of this project area include: 1) improving the scientific underpinnings of our understanding of the glass transition event and its kinetics; 2) providing fundamental data on the variation of the viscoelastic properties of polymers due to structural recovery (physical aging); 3) expanding the knowledge base for aging to include the effects of moisture and polymer crystallinity on material performance.

\section{Accomplishments:}


Time-Temperature-Aging Time Superposition and Volume Recovery in Polycarbonate Glasses

Time-temperature and time-aging time superposition data have been obtained for a commercial grade polycarbonate. For time-temperature superposition the results show the deviation of the sub- $\mathrm{T}_{\mathrm{g}}$ response from the WLF equation. Two response regimes are observed: at temperatures far below $\mathrm{T}_{\mathrm{g}}$ the $\log \left(\mathrm{a}_{\mathrm{T}}\right)$ is linear in $\mathrm{T}$, followed by a transition towards the WLF behavior as $\mathrm{T}_{\mathrm{g}}$ is approached. The temperature at which the behavior changes from a linear dependence of $\log \left(\mathrm{a}_{\mathrm{T}}\right)$ on $\mathrm{T}$ to the transition-type behavior is found to depend on the aging time. This temperature decreases as aging time increases.

The time-aging time response is found to behave in a normal way, i.e., the viscoelastic responses of the polycarbonate obtained at different aging times $t_{c}$ can be superimposed by a shift $a_{t e}$ along the frequency (time) axis. At temperatures far below $T_{g}$ the $\log \left(a_{t e}\right) v s \log \left(t_{e}\right)$ is constant and has a slope somewhat less than unity. However, nearer to $T_{g}$ the slope decreases and there is a second regime in which the aging virtually ceases. In this polycarbonate no aging is observed above $136.9{ }^{\circ} \mathrm{C}$.

This part of the NIST collaborative project with the GM/GE Thermoplastic Engineering Design program is providing the data generated here for dissemination within the TED program and to incorporate into our internal computer codes for estimation of dimensional stability of post-molded plaques. Additional data are being generated for volume recovery that will be important both for comparisons of the aging behavior with the volumetric response and for use in modeling of sub- $\mathrm{T}_{\mathrm{g}}$ dimensional stability and residual stresses in postmold applications.

\section{Physical Aging in Nylon 66}

As part of a CRADA with the E.I. DuPont de Nemours Company and the University of Delaware, the capabilities of the laboratory as a center of expertise in the physical aging of amorphous polymers were extended into the area of semi-crystalline polymers using nylon 66 as the material for investigation. The experimental program set out to determine: 1) how nylon ages in the temperature range just below the glass transition of the amorphous phase, where normal aging behavior is expected; 2) how aging occurs in the regime just above the calorimetrically determined glass transition; 3 ) how aging occurs in the regime above the glass transition determined by dynamical mechanical measurements.

The physical aging process of nylon 66 was investigated over a range of temperatures from below the calorimetrically determined glass transition to well above it. It was found that the classical time-aging time superposition was valid excepting for some samples exposed to both higher temperatures $\left(55-65^{\circ} \mathrm{C}\right)$ and high stress $(24 \mathrm{MPa})$. The double logarithmic shift rate, $\mu$, was found to depend on temperature and stress level. It was found that, unlike many amorphous polymers, time-temperature and stress-temperature superpositions were not possible for the nylon 66 data. Furthermore, the polymer could not be aged into equilibrium 
even at temperatures above the nylon's typical glass transition values of $46-57^{\circ} \mathrm{C}$ reported in literature. Dilatometric measurements show higher volume recovery rates for the semicrystalline nylon than observed for amorphous polymers. Furthermore, the temperature dependence of the volume recovery rate is opposite from that observed for the amorphous polymers, i.e., it increases in going from below to above the calorimetric glass transition. This indicates that some of the aging in the nylon must be attributed to changes in the crystalline phase.

The results of the study were communicated to the technical community and to DuPont. Two articles describing the work were produced. In addition, the work has lead to a renewal of the DuPont CRADA to expand the study into films of polyethylene terephthalate.

Fracture Incubation in Engineering Thermoplastics

In a CRADA with the Dow Chemical Company, experiments were undertaken to characterize the creep, creep rupture and and fracture incubation processes in polycarbonate (PC) and syndiotactic polystyrene (sPS). A lever arm creep stage working with dead load has been developed to perform creep and creep rupture experiments at room temperature. First experiments to determine the time to failure behavior of a low molecular weight polycarbonate produced by DOW, were performed on two samples different in the thermal history. The failure time decreased dramatically upon annealing for one hour above $T_{g}$ and aging one week at room temperature. Future work aims to map out the failure response of the polymers of interest in order to determine the range of stresses and times in which to perform creep rupture experiments on the SAXS facility. The SAXS experiments on specimens under load are used to measure the size and volume density of sub $\mu \mathrm{m}$ size 'protocracks'. Preliminary SAXS experiments indicate that the polycarbonate shows very little scattering in the q-range in which proto-cracks are expected to be detected. Also, the sPS material shows an anomalous power law behavior in the q-range between $0.2 \mathrm{~nm}^{-1}$ and 0.5 $\mathrm{nm}^{-1}$ which requires further investigation. To perform these experiments the deformation stage used for the SAXS equipment has been improved to enable one to perform creep experiments up to $80 \mathrm{~kg}$.

The viscoelastic and creep rupture behaviors of polymers have been approached from a variety of different viewpoints. Molecular considerations lead to kinetic and reaction rate models of time dependent failure in polymer glasses. These models provide a useful description of the failure behavior of polymers which are subjected to static and monotonically increasing loading. However, they have been less successful in describing failure when the loading history becomes more complicated. In relaxation and creep experiments it is found that curves at different strain (stress) levels can be superposed to form a mastercurve by a shift factor along the log time axis. The concept that the applied stress simply shifts the viscoelastic spectrum has been used to develop descriptions of the nonlinear viscoelastic response of polymer glasses under constant loading history.

Ultimately, it is expected that these approaches can be brought together in a coherent picture of the influence of aging and its associated changes in viscoelastic properties on the failure 
response of polymers.

The Aging Response of Polymeric Glasses in Relative Humidity Jump Experiments

Dimensional stability and residual stresses in polymers and composites have largely been treated as being determined by the thermal expansivity of the resin when subjected to thermal histories, particularly cooling through the glass transition range. On the other hand, it is well known that epoxy resins absorb considerable moisture and this can be a cause of significant dimensional change and reinitiation of the physical aging process due to plasticization. The object of this study is to establish the validity of the hypothesis that moisture acts as a simple plasticizer in epoxies and, consequently, changes in moisture content in polymer glasses can be treated in a similar way as changes in temperature.

Here we have performed experiments using model di- or tri-amine terminated polypropylene oxide/ DGEBA epoxy systems. Samples are subjected to a constant humidity and temperature $\left(\mathrm{RH}_{1}\right.$ and $\left.\mathrm{T}_{1}\right)$ environment until sample mass equilibrium is attained. The samples are then moved to a tensile testing machine equipped with a constant humidity oven set at the same temperature but a different relative humidity $\left(\mathrm{RH}_{2}\right)$. The experimental methodology treats the change in relative humidity (RH-jump) in a manner similar to a change in temperature. Consequently, subsequent to the 'quench' (RH-jump) the changing viscoelastic response in uniaxial creep at different times after the 'quench' can be used to probe the aging response. While the process is complicated by the long diffusion time of the moisture into $\left(\mathrm{RH}_{1}<\mathrm{RH}_{2}\right)$ or out of $\left(\mathrm{RH}_{1}>\mathrm{RH}_{2}\right)$ the sample, obvious aging effects are observed, similar to those observed in temperature jump experiments. The results demonstrate the importance of understanding the coupling between moisture ingress and egress in epoxies and the physical aging process. These preliminary results were presented at the International Conference on Composites Engineering in August, 1994.

The Impact of Physical Aging on Craze Initiation and Propagation in Polymers

The investigation of the effects of physical aging on the crazing behavior of glassy polymers should yield better estimates of polymer lifetime. Improved estimates of polymer lifetime help in the selection of particular polymeric materials for particular applications.

Furthermore, a better understanding of the interaction between aging and crazing may lead to improved methods of processing or pre-treatment of polymeric materials to obtain longer polymer lifetimes.

Preliminary work has resulted in the development of an experimental capability to perform crazing experiments in uniaxial creep and stress relaxation conditions and in equibiaxial stress conditions.

\section{Viscoelastic Behavior of Polymers:}


G.B. McKenna, M.Y.M. Chiang, W.K. Waldron, Jr., C.R.Schultheisz, J. Niemiec, C.L. Schutte, J.-J. Pesce ${ }^{1}$, J. Beckmann ${ }^{1}$ and H. Chai ${ }^{2}$

${ }^{1}$ Institut Charles Sadron (EAHP), Strasbourg, France

${ }^{2}$ Tel-Aviv University, Tel Aviv, Israel

\section{Objectives:}

In this project the constitutive framework is developed within which to model residual stresses, dimensional stability, and performance of engineering plastics in post-processing conditions, e.g., post-injection molding. Currently under development is 'Solid Rheology' for the sub-yield behavior of solid-like polymers based on 'material clock' constitutive models that shows considerable promise for material description and for reasonably easy incorporation into finite element codes.

\section{Accomplishments:}

Nonlinear Viscoelasticity and Dimensional Stability in Engineering Polymers

This project complements the NIST ATP Program entitled "Engineering Design with Injection-Molded Thermoplastics" with the General Motors Corporation and General Electric Company. The overall goal of this ATP program is the development of "design-to-build" methodologies for injection molded parts. Several critical areas related to this goal are required. First, there is a need to accurately predict the dimensions of parts when they first leave the mold so that they can be successfully mated to other parts. Second, the ability to predict the stability of the parts during subsequent processing and service conditions is necessary. In addition to physical aging, such factors as high temperature manufacturing steps, such as encountered in the paint bake cycle, and long term exposure to seasonal temperature variations need to be considered. Third, the ability to model the nonlinear response to the mechanical histories associated with processing and service conditions is needed. Actually, the last two phenomena are coupled, i.e., thermal histories may create residual stresses and mechanical deformations may recover at elevated temperatures. The current objective is to survey the thermodynamic and constitutive theories and identify the most practical methods of describing the nonlinear response of polymers to the thermomechanical histories typical of industrial processes.

The effort has been divided into three areas:

1. Simulation of Yield in Compressible Solid Polymers. A constitutive equation with a shear strain and volume clock is presented for a compressible nonlinear viscoelastic material. The constitutive equation is used to simulate the finite circumferential deformation of a hollow cylinder which is perfectly bonded to a rigid support at its inner surface and a rigid shell at its outer surface. The nonhomogeneous problem is solved for two load conditions: (1) moment control where the outer surface is subjected to a constant moment rate and (2) rotation control where the outer surface is caused to rotate at a constant rate. In each case, the cylindrical surfaces rotate about the central axis and move radially, thereby inducing a radial shear strain and volume change distribution. It is shown that the particle volume distribution depends on the material parameter $f$ and there is a time when a layer of large 
shear strain gradient near the inner surface begins to grow rapidly indicating yield-like behavior. For rotation control, the applied moment necessary to maintain the prescribed rotation and the corresponding shear stress histories can have local maxima even with unenhanced stress relaxation for certain values of material parameter $f$.

2. Experiments and Analysis of the Nonlinear Viscoelastic Response and Apparent Rejuvenation of an Epoxy Glass. A modified BKZ theory with a strain dependent physical aging clock is used to describe the nonlinear viscoelastic response and apparent rejuvenation of an epoxy glass. In particular, the model is used to describe the results of aging experiments similar to those of Struik (1978). In one type of experiment, after a quench from above to below $T_{g}$, the sample is subjected to a series of constant "probe" strains and time-aging time superposition is used to determine the shift factor $\mathrm{a}_{\mathrm{e}}$ history. If a single large strain probe is applied within a series of small probes, a large decrease in $\mathrm{a}_{\mathrm{c}}$ is observed followed by its rapid increase in the subsequent small probes. Experiments in our torsional dilatometer reproduce these essential features and the results are compared with predictions of the modified BKZ model and discussed in terms of the Struik's erasure of aging hypothesis.

3. Modeling the Torsional, Normal Force, and Volume Relaxation of an Epoxy Glass in the Nonlinear Viscoelastic Range. Data from Duran and McKenna's(1990) experiments using an automated mercury torsional dilatometer are used to determine the strain potential function of a model epoxy glass. This instrument takes readings of torque, normal force, and volume change upon the application of a twist at one end of the sample. In earlier work, the measured torque and normal force were fit to separate single step relaxation functions. These single step relaxation functions were then used to predict the response to multiple step histories using the BKZ constitutive equation for an incompressible elastic fluid. This approach has two deficiencies. First, the assumption of incompressibility precludes the volume changes which are measured experimentally, and second, the deformation of the epoxy is limited by chemical crosslinks which make a fluid model inappropriate. In the current study, we use the isochronal torque, normal force, and volume change measurements of Duran and McKenna to determine a time dependent strain potential function. A polynomial representation of the strain energy density function is assumed and a material identification method is outlined for the determination of the polynomial constants from the torsional data. We show that although the scaling methods are not valid for compressible materials, they provide good estimates for the form of the strain energy density function.

\section{Evaluation of Material Clock Models for the Nonlinear Viscoelastic Characterization of Polymer Glasses}

Polymer forming processes are complex and impart residual thermal and mechanical stresses into parts. Furthermore, the material properties of polymers change continuously with time after formation due to physical aging processes. The search for more efficient design and manufacturing methods for polymer part dimensions and performance is leading to increasing needs for material models and data that can be used in computer simulations of both the 
manufacturing and end use environments. Of particular interest here is the non-linear viscoelastic response of a glassy polymer below the yield point, the description of which ultimately influences the evolution of part dimensions in, e.g., injection molding.

A study of the nonlinear viscoelastic response in torsion of a polycarbonate resin in the glassy state is being carried out. We have introduced a nonlinear viscoelastic model material response based on an extension of the Bernstein-Shokooh stress clock version of the BKZ theory of an ideal elastic fluid. The material clock or pseudo-time was chosen to depend on the strain energy in the material--hence, an energy clock function. Single step and two-step stress relaxation experiments were used to test the energy clock model. The original BKZ and a modified Boltzman equation were also compared with the data. The second step torque response was reasonably described using the energy clock model for the history in which $\psi_{2}=\psi_{1} / 2$ and for which $\psi_{2}=2 \psi_{1}$. In the case in which the deformation returns fully to zero $\left(\psi_{2}=0\right)$, however, the torque response was reasonably described by both the original BKZ model and the modified Boltzman model. The energy clock model, however, greatly overestimated the magnitude of the negative torque obtained upon reaching zero deformation. A systematic evaluation of material clock models for the evaluation of the nonlinear viscoelastic response of solid-like polymers is currently taking place in our laboratories and other clock models will be evaluated in the future.

Torsional Dilatometric Evidence of the Coupling Between Aging and Mechanical Response in Polymer Glasses

The development of nonlinear constitutive equations for the description of the response of solid-like polymers is an important facet in building predictive methodologies for performance of polymers in engineering applications. Having a sound physical basis for building such equations is an important goal. In the case of glass forming polymer there is controversy over the nature of their nonlinear viscoelastic response and how it is influenced by the structural recovery and how the structural recovery is impacted by large deformations. Solution of the problem determines part of the structure that nonlinear constitutive laws take.

Dilatometric experiments using the NIST torsional dilatometer show that, while the volume recovery of a polymer glass subsequent to a temperature jump is accompanied by changes in the viscoelastic response (physical aging), the underlying (baseline) volume recovery is unaffected by the large mechanical deformations that are applied. This contradicts the rejuvenation hypothesis for nonlinear viscoelastic behavior of glasses and has important implications for the development of nonlinear constitutive equations. In particular, it implies that the viscoelastic response depends upon the immediate (though evolving) glassy structure or thermodynamic state. However, the immediate glassy structure is, in some sense, independent of the applied stresses. This means that a constitutive law can be written that depends upon the thermodynamic state without being coupled to a nonlinearity induced by the state itself depending on the applied stresses. In other words, the mechanical portion of the thermodynamics depends on the underlying state of the glass, but the state of the glass and its evolution are decoupled from the applied stresses. 


\section{Viscoelastic Behavior in Polymer Engineering Through Finite Element Analysis}

Polymeric materials are primary candidates for many engineering applications in components and structures for automobile and electronic packaging industries. Production of reliable components and structures requires an ability to design not only so that initial property requirements and dimensional tolerances are met, but also that these properties and dimensions are maintained throughout the service lifetime and environment. Also, it must be noted that the dimensional tolerances and mechanical properties are so interrelated that they can not be considered independently. Therefore, the ability to predict and control the dimensional changes during the manufacturing process and assembly of components becomes very critical. In this project the primary objectives are:

(a) To develop and incorporate fundamental concepts of material clock models in viscoelasticity into numerical models (finite element method)

(b) To illustrate the essential influences of material viscoelasticity on part performance through solution of typical engineering problems (such as dimensional stability of structures)

In the current work we have chosen to model the dimensional response of a simplified model of a composite inner layer typical of a printed wiring board (PWB). A simplified micromechanical model has been used in a linear thermo-viscoelastic finite element analysis to examine the role of material viscoelasticity (stress relaxation or creep) on dimensional changes. The analysis incorporates material properties determined in our laboratory for the thermo-viscoelastic response of an epoxy resin. The model, based on a unit representative cell and classical laminate theory, serves to demonstrate the time-dependency of the inner layer dimensions during the cooling process after the part is removed from the mold. Long term behavior is explored in a numerical simulation which includes stress relaxation below the glass transition temperature $\left(\mathrm{T}_{\mathrm{g}}\right)$. In this model, the relaxation response at a given temperature, $\mathrm{T}$, is described by substituting a temperature-dependent reduced time factor into a formerly temperature-independent relaxation function (time-temperature superposition, TTS). Work is now beginning to incorporate these concepts and those involved in nonlinear viscoelasticity into a model for an injection molded plaque.

The work has shown the importance of accounting for the effect of time-dependent stress responses on dimensional change after the part is removed from the mold. Incorporation of a realistic time-temperature superposition (TTS) response at temperatures below $T_{g}$ shows that these changes continue for long times after reaching ambient temperature. In addition, preliminary results show that the value of the coefficient of thermal expansion (CTE) of the inner layer depends on thermal history during the measurement, and the final configuration of the inner layer is governed by the stress-free state of the substrate. Finally, these results show that dimensional stability can be dramatically affected by the thermal history of the material.

Characterization of Failure of Polymeric Interlayers Subjected to Shear Loading 
The mechanical properties (i.e. stress-strain relationship) of thin polymeric interlayers are of great interest in a variety of technological applications, including traditional adhesive bonding, microlamination, interlaminar fracture of composites and electronic packaging. Most mechanics treatments of laminated materials either neglect the effect of the interlayer or assume that it behaves like the bulk material. However, recent experiments show that the mechanical properties of the interlayer may greatly depend on its thickness. For example, it was found that the ultimate shear strain of a brittle epoxy increases from several percent to over $300 \%$ upon decreasing the bond thickness from $0.1 \mathrm{~mm}$ to several micrometers. Therefore, in this project, the primary objectives are:

(a) To incorporate material and geometrical nonlinearity in a finite element model to predict the deformation characteristics at the crack tip of an adhesive interlayer

(b) To compare the model predictions with experimental data on the global response of an adhesive interlayer

(c) To establish a local material failure criterion, which is intrinsic and independent of bond thickness for the adhesive interlayer

In this project, the finite element code ABAQUS was employed to obtain the large-strain elastoplastic stress state in the adhesive for the End-Notched Flexure (ENF) and End-Loaded Split (ELS) specimens. These specimens are commonly used for evaluating the mode II fracture toughness of laminated composites and adhesively bonded joints. In addition, their relative simplicity provides insight to the understanding of fracture in more complex adhesive bonding configurations. Experiments show that the adhesive thickness, $t$, plays a major role on the fracture conditions in the bond. Therefore, $t$ was set as a variable in this study, ranging from $18 \mu \mathrm{m}$ to $0.4 \mathrm{~mm}$. Global quantities such as plastic zone length and across-thebond average shear strain along that zone are compared with experimental data as well as with a previously developed shear-lag analysis in our laboratory. The applicable range of the shear-lag model is assessed from comparison with finite element results for various slenderness ratios of the split beam. In addition, some general observations concerning the local behavior at the crack tip are made; a more exhaustive investigation of this is deferred to a future work. The majority of elastoplastic stress analyses of adhesively bonded joints treat the adhesive interlayer in accordance with the $\mathrm{J}_{2}$ yield criterion. However, it is well known that for polymeric adhesives, which are of particular interest in this work, the yield conditions depend on pressure and follow closely a modified Mohr-Coulomb yield criterion. For this reason, the latter type of material model was also included in this study. 


\section{POLYMER CHARACTERIZATION PROGRAM}

\section{Goals}

The Polymer Characterization program provides measurement methods, data, and standard reference materials needed by U.S. industry, research laboratories, and other federal agencies to characterize polymers for processibility, properties, and performance. Molecular weight and molecular weight distribution are the molecular characteristics of polymers that most affect their processing, properties and performance. Properties and performance may also vary widely depending on the solid state structure formed during processing. Therefore, the focus of the program is on techniques that measure molecular weight, its distribution and the solid state structure of polymers. Primary methods employed for molecular weight are dilute solution light scattering and osmometry; chromatographic techniques, which require calibration by standards of known molecular mass, provide information on molecular weight distribution. Spectroscopic methods, solid state nuclear magnetic resonance (NMR) and infrared, and $\mathrm{x}$-ray diffraction are developed and applied to elucidate the solid state structure of polymers.

The polymer industry and standard organizations assist in the identification of current needs for standard reference materials. Based on these needs research on characterization methods and measurements are conducted leading to the certification of standard reference materials. Standards are produced for calibration of gel permeation chromatographs, the principal method used by industry for assessing molecular weight and molecular weight distributions, and melt flow standards that are used in the calibration of instruments used to determine processing conditions for thermoplastics.

\section{FY-94 Significant Accomplishments}

- An apparatus was constructed to purify by recrystallization polyethylene fractions which will be certified for molecular weight.

- A publication reported theoretical studies that support experimental work showing that chromatography of block copolymers at the adsorption transition of one of the blocks reveals the chromatogram of the other block.

- $\quad$ NMR studies of ethylene vinyl alcohol copolymers revealed that at concentrations where the vinyl alcohol units are guests in the polyethylene lattice the concentration of vinyl alcohol groups in the lattice is identical to the overall concentration.

- $\quad$ Proprietary research was conducted on the Division's small angle x-ray facility by scientists from GE, Mobil Chemical, W.R. Grace and Armstrong World Industries. 


\title{
$\underline{\text { Standard Reference Materials }}$
}

\author{
C.M. Guttman, J.R. Maurey, W.R. Blair and B.M. Fanconi
}

\section{Objectives:}

Provide the U.S. polymer industry with standards for calibration of instruments used in the control of the synthesis and processing of polymers. Principal polymer standards are certified for molecular weight and melt flow rate; the former are used to calibrate gel permeation chromatographs and the latter to calibrate melt flow indexers.

\section{Accomplishments:}

Aqueous SEC Molecular Weight Standard

No standard reference materials (SRM's) are presently available for water-soluble high polymers which are industrially important in such areas as adhesives, coatings, paints, lubricants and oil resistant plastics. The need for such standards has been established through consultations with industrial scientists, by market surveys and in the recommendations of the Polymers Division Assessment Subpanel.

By far, the most pressing need for molecular-weight certified water soluble polymers is in the calibration of aqueous size exclusion chromatography (SEC). Polyethylene oxides were selected for a series of molecular weight SRM's to meet this need based on their usage on most aqueous SEC columns without special column conditioning .

Absolute molecular weight measurements have been completed on two narrow molecular weight distribution polyethylene oxides, SRM 1923 with a Mw of 27,000 and SRM 1924 with a Mw of 127,000 . A report on the certification and use of these SRM's has been completed.

\section{SRM 1475 Recertified}

SRM 1475, a polyethylene molecular weight standard commonly used in SEC calibration and as a melt flow standard was recertified. The SEC measurements on the new materials were compared to those of the previous ones to evaluate consistency. The melt flow rate measurements on SRM 1475 were completed and a report on SRM 1475 was prepared consistent with the new NIST requirements on reporting uncertainties in measurement results.

\section{New Polyolefin Molecular Weight Standards}

Polyolefins, in particular polyethylenes, are the most widely used commercial polymers. High temperature $\left(150^{\circ} \mathrm{C}\right)$ Size Exclusion Chromatography (SEC), although a relative method requiring calibration, is the most commonly used method to establish the molecular weight of these polymers. Yet, few SEC calibration standards are available on the market for the calibration of high temperature SEC. Molecular weight fractions with $\mathrm{Mw} / \mathrm{Mn}$ of 
less then 1.2 are most useful materials for calibrating SEC.

To our knowledge there is only one commercial supplier of sharp fractions of polyethylene. These materials are a repackaging of the NIST one gram polyethylene fractions, SRM 1482, SRM 1483 and SRM 1484, into 0.25 gram samples. The lack of commercial standards arises out of the difficulties obtaining sharp molecular weight fractions of these materials and in measuring absolute molecular weights by light scattering or osmometry at temperatures as high as $150 \mathrm{C}$. In the past, NIST had a commercial polyethylene fractionated by an outside contractor and from three of the fractions produced SRM 1482,1483 and 1484.

SRM 1484, a sharp fraction polyethylene of molecular weight 120,000 dalton, has recently been recertified. At the current sales rate of this SRM, this material will be exhausted in a few years. There is no more of the original 120,000 dalton material.

Some cuts of the material that was fractionated in the original development of SRM 1482, SRM 1483 and SRM 1484 remain. From these fractions it appears that sufficient quantities are available with molecular weights around $160,000,70,000$ and 5000 dalton to produce three additional narrow fraction polyethylene SRM's.

One of the new fractions has been purified by recrystallisation. The others will be treated similarly. Certification of the molecular weight and intrinsic viscosity of all three fractions will begin in FY95. These new materials along with current SRM 1482 and 1483 provide a set of polyethylene fractions having molecular weights of

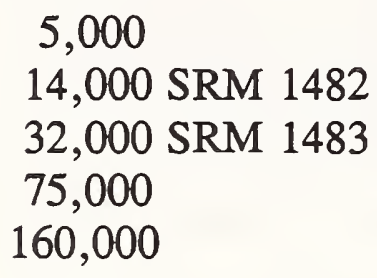

dalton. This will provide the polymer industry with an adequate set of molecular weight calibrants for polyethylene. A higher molecular weight calibrant is also needed, but at this time no method is available to produce one.

This work is supported by SRMP.

\section{Chromatographic Techniques for Polymer Characterization}

C.M. Guttman, W.R. Blair, E.A. DiMarzio, J. Douglas and S. Chou

\section{Objectives:}

This project addresses the need to develop characterization techniques for molecular weight distributions of copolymers. Theoretical and experimental works aims at providing industry with a simple chromatographic method to obtain the molecular weight distribution of a single 
block of a diblock or triblock copolymer. The approach is to develop theory to explain and analyze data from chromatography near the polymer absorption transition and determine the absorption transition for various polymers in mixed solvents to obtain a variety of homopolymer systems which can be used to analyze their copolymer. Associated work models the affects of strong polymer-substrate bonds on adsorption characteristics.

\section{Accomplishments:}

Chromatography of Polymers at the Adsorption Transition

The chromatography of a polymer from the exclusion region (Size Exclusion Chromatography) into the adsorption range is modeled for chains with a variety of architectures and interactions with surfaces. Using the matrix method, arbitrary energies of adsorption of the individual monomers for the surface can be treated. Chains with internal stiffness energies and architectures, including combs, stars and rings can be modeled. The modeled polymer molecules may be composed of several species to represent arbitrary copolymers. Theoretical studies support the experimental work by Pasch showing that diblock copolymers at the transition point of one of the blocks give chromatograms which display the SEC of only the other block. The chromatography of triblock copolymers at the transition point has been modeled. Because of its generality, the method promises to be of great utility in interpreting elution experiments on macromolecules of various architectures.

\section{Effect of Heterogenous Energies on Absorption Properties of Polymer Chains}

The presence of a few strong bonds in a chain with many weak bonds can change the properties of the polymer dramatically. For example, the presence of a few strong bonds will allow incompatible polymers to be compatible in a polymer blend. In recent experimental work related to the dilute solution characterization of polymers intended for use as an SRM the association properties of the polymers were found to differ significantly due to the change of a few groups in the backbone. Currently, the interaction of polymer with a few strong interactions with a surface is being studied by both numerical and analytical methods.

\section{Structural Characterization of Polymers by NMR}

\section{D.L. VanderHart and S.J. Kryder}

\section{Objectives:}

NMR, a powerful characterization technique, is further developed and used to elucidate the molecular and microstructural level features that control or determine physical properties of polymers. Areas of focus include: exploration of multipulse proton spin diffusion techniques in solid polymers, use of ${ }^{13} \mathrm{C}$ NMR in the characterization of liquid crystal polymers, examination of the partitioning of comonomers into crystal lattices, and studies of the crystal structure of cellulose triacetate and its oligomers. 


\section{Accomplishments:}

Exploration of Multipulse Proton Spin Diffusion Measurements for the Characterization of Solid Polymers

NMR measurement techniques are developed that will yield information about small-scale $(2-100 \mathrm{~nm})$ spatial inhomogeneity in polymers. Moreover, in the event that chemical inhomogeneity is also present, another objective is to develop methods by which simultaneous information about chemical inhomogeneity can be obtained. Examples of inhomogeneities amenable to study are: a) polymer blends (intimacy of mixing in compatible blends, domain size and some information on domain stoichiometry in phase separated blends), b) size of crystalline and non-crystalline domains in semicrystalline polymers, c) spatial distribution of motional heterogeneity in glassy polymers (what is the minimum distance representing the sample-averaged 'free volume' in a glassy polymer?), and d) spatial distribution of motional heterogeneity in high strength fibers (Is motion confined to the outer molecular layer of a microfibril, or is there evidence of internal defects?).

In these NMR studies spin diffusion techniques are combined with two other techniques, namely, multiple-pulse (MP) line narrowing techniques and magic angle spinning (MAS). The simultaneous application of MP and MAS achieves a very modest (compared to liquidstate NMR) amount of chemical shift resolution. In polymer blends, the resolution is often sufficient to yield a contrast in the spectra of two polymers, hence one can differentiate the signals arising from each component and deduce information about the phase dimensions. As part of this program, other methods, such as differential relaxation under MP, are investigated to establish polarization gradients based on differential molecular mobility. These experiments form the basis of the investigations into motional heterogeneity in glasses and defect structure in the interior of crystalline high-strength microfibrils. To aid in the data interpretation calculations are performed to relate spin diffusion with different initial conditions and morphologies.

Most current work concerns polymer blends and utilizes multiple-pulse techniques which require more experimental attention than other NMR techniques might require. The sensitivity of protons combined with the modest resolution achievable via multiple pulse methods make this technique widely applicable to the study of polymer blends. The measurement technique shows considerable promise for polymer blends, especially for those blends of glassy, high- $\mathrm{T}_{\mathrm{g}}$ materials, where thermal history, kinetics and thermodynamics often dictate the level of mixing and the resulting domain sizes.

Outputs of the work to date are a method for characterizing blends and a non-destructive way of obtaining information about phase stoichiometry. The latter provides insight into the thermodynamic compatibility of certain blends which, by their nature, cannot form under conditions of thermodynamic equilibrium. Blends of cellulose with poly(4-vinyl pyridine) and with poly(acrylonitrile) can be produced with domain sizes in the range of $3-12 \mathrm{~nm}$; yet, the phase separation which occurs produces near-pure phases, indicative of the fundamental incompatibility of these polymer pairs. The practical significance of obtaining 
information about thermodynamic compatibility is that predictions about the stability of the morphology of these materials and relationship between slow phase separation and concomitant changes in materials properties can be made. This is difficult information to obtain in any other way.

Current work on glassy polymers and high strength fibers aims to improve the understanding of the inhomogeneous character of glassy polymers and of the structural perfection of high strength fibers. Knowledge in this area should be useful in attempts to bridge the gap between molecular organization and mechanical properties, especially relative to glasses.

\section{${ }^{13}$ C NMR Study of Backbone Smectic Polymers}

In joint work with Dr. Ernesto Pérez, Instituto de Ciencia y Tecnología de Polímeros, Madrid, NMR is combined with DSC and x-ray diffraction to characterize a new class of smectic, liquid-crystalline (LC) polymers. These polymers are polyesters having bibenzoate groups in the polymer backbone as the mesogen and flexible spacers in the backbone consisting of aliphatic moieties. The emphasis is on certain characteristics which might influence the usefulness of these materials, such as a) degree of crystallinity and polymorphism, b) the size of crystalline domains, c) the persistence of LC order in the noncrystalline region of a partially crystalline sample, and d)the level of chain entanglement in the LC state relative to the glassy non-crystalline state.

Findings of interest include a) differences of mobility between the spacer and the mesogen in the LC phase (as expected, the mesogenic group is much less mobile than the spacer), b) only slight modifications to the mobility of the non-crystalline segments in the semicrystalline samples compared with the mobility of the LC segments (this supports the notion that the non-crystalline chains retain much of their LC order), c) evidence for polymorphism in the crystalline regions for poly(triethylene glycol p,p'-bibenzoate) where this polymorphism seems to arise from different conformations of the spacer, and d) evidence that chains do not have to disentangle significantly in order to form the LC and, as a consequence, low degrees of crystallinity upon crystallization. (Thus, processing from a smectic LC precursor will probably not result in a material of high crystallinity; moreover, expectations for a high degree of orientation are also tempered.)

\section{Partitioning of Guest Comonomers into Crystal Lattices}

The purpose of this work is to provide experimental data so that a more intelligent tailoring of materials properties of copolymers may emerge via control of comonomer levels and processing. Aspects of the work are as follows: a) establish and promote a ${ }^{13} \mathrm{C}$ NMR method, applicable to copolymers, for measuring the concentration of minor-fraction comonomer repeat units in the crystalline regions where the host lattice is that of the majorfraction comonomer, b) classify comonomers according to the degree to which they are excluded from the crystal, c) measure the influence of comonomer exclusion on the level of crystallinity which can be developed, d) obtain the dependence of comonomer concentration 
in the crystalline regions on overall comonomer concentration, and e) determine the time dependence of comonomer concentrations in crystallites in a given sample as a mechanism for degradation of properties.

Materials investigated in this work are ethylene vinyl alcohol (EVOH) copolymers and bacterially synthesized, highly isotactic copolymers of poly(3-hydroxybutyrate-co-3hydroxyvalerate) (PHB/V). The latter is a biodegradable copolymer with reasonable mechanical properties; however, it has a tendency to age with time and develop brittleness. For the PHB/V copolymers of lower HV content, where the host lattice is PHB, it is found that a) the HV residues are preferentially excluded from the crystalline regions and over the range of overall $\mathrm{HV}$ mole fraction from 0.044 to 0.27 , the concentration in the crystalline region ranges from 0.015 to 0.20 indicating that the crystalline region concentration is not a linear function of overall concentration. An explanation is that the more comonomer units reside in the crystalline region, the more the lattice of the host is distorted which reduces the energy barrier to incorporation of additional comonomers into the crystalline region. b) There is evidence that at higher HV concentrations, e.g. a mole fraction $\mathrm{HV}$ of 0.27 where the PHB lattice still dominates, a distribution of compositions of $\mathrm{HV}$ exists throughout the various crystallites. In fact, a small fraction of crystals characteristic of the PHV lattice form. c) There is little effect of comonomer exclusion on the overall crystallinity of these samples (albeit the heat of fusion is a function of the comonomer concentration).

In contrast to the $\mathrm{PHB} / \mathrm{V}$ copolymers, the $\mathrm{EVOH}$ copolymers, in the range where the $\mathrm{VOH}$ residues are guests within the polyethylene lattice, show almost no partitioning, i.e. the concentration in the crystalline regions is very close to the overall concentration. This consistent pattern over a range of concentrations suggests that hydrogen bonding between $\mathrm{VOH}$ residues is not an important factor in the level of incorporation. Rather, it seems that the hydroxyl group is not much different from a proton in the way it fits into the lattice. An interesting further observation is that short-chain (mostly butyl) branches, are rejected from the crystalline regions, even though the hydroxyl groups are incorporated. Hence, it is the short chain branches and not the density of hydroxyl groups which limit crystallinity in these materials.

Characterization by NMR and Raman Spectroscopy of Cellulose Triacetate and its Oligomers

Cellulose triacetate (CTA) is an important commercial polymer, especially in the form of films. Its high $T_{g}$ gives good dimensional stability and it is also very transparent. This is a semicrystalline material; moreover, CTA shows polymorphism, although the corresponding crystal structures have not been elucidated completely. Goals of this work were to a) establish similarities in crystal structure of CTA oligomers relative to allomorphs of the CVA polymer. With this information, the structure of the CTA polymer could be inferred from Xray crystallographic studies of oligomers for which single crystal specimens can be readily prepared, b) study the influence of solvents, preparative history, and thermal processing on the crystalline state of CTA, c) compare NMR results with characteristics postulated from xray studies of CTA allomorphs, d) relate observations regarding CTA and its allomorphs to 
cellulose chain polarity. Cellulose also shows polymorphism and a strong correspondence exists between the allomorphs of cellulose and those of CTA prepared by either heterogeneous acetylation or saponification.

Results are summarized as follows: a) oligomers, from the pentamer through the nonamer (the largest oligomer studied in the present work), show both NMR and Raman spectra strongly related to the CTA(I) lattice, b) heterogeneous acetylation of cellulose I-type materials leads to CTA(I) materials but this CTA(I) crystalline form is only evident after heat treatment at $220^{\circ} \mathrm{C}, \mathrm{c}$ ) heterogeneous acetylation of highly non-crystalline cellulose gives rise, after heat treatment, to some rather poorly developed CTA(II) crystallinity, d) CTA dissolved in diphenyl ether or partly dissolved in dibenzyl ether yields well developed CTA(II) crystallinity after precipitation and heat treatment, e) CTA(I) and cellulose (I) have parallel chains whereas CTA(II) and cellulose (II) have antiparallel chain character. These conclusions are partly based on NMR observations that CTA chains are very sluggish in their ability to rearrange and develop crystallinity which suggests that the resultant CTA allomorph reflects the dominant local statistics of chain polarity. The only time CTA(I) forms is when cellulose (I) crystals are precursors and when heterogeneous acetylation has taken place so as to preserve the local chain polarity. When the precursor state of CTA is a random arrangement of CTA chains, e.g. when non-crystalline CTA is the precursor, then only CTA(II) forms because of the low probability of finding local bundles of parallel chains.

There are two impacts of this work: a) the groundwork has been laid for solving the crystal structure of CTA(I) via the growth of a single crystal of an oligomer of CTA and b) if the arguments about chain polarity in both CTA and cellulose are correct, then the need to convert chains from parallel to antiparallel character in the industrially significant conversion of cellulose to rayon will probably limit the cellulose precursor materials to those with smaller lateral crystallite dimensions. For example, wood cellulose and cotton have smaller lateral crystallite dimensions relative to celluloses derived from certain bacteria or algae; hence, mercerization of the wood or cotton cellulose should be easier assuming a modest probability that a pair of adjacent microfibrils have opposite chain polarity.

\section{Transport of Small Molecules in Glassy Polymer Matrices}

\section{D.L. VanderHart, R.J. Schadt and S.J. Kryder}

\section{Objectives:}

The principal objective is to set up facilities to characterize the behavior of small molecules in glassy polymers. Solid-state proton NMR techniques are utilized to better understand the transport of water through epoxies and epoxy-fiberglass composites. This system was selected based on the known deleterious effects of water on the mechanical properties of epoxies and their fiberglass reinforced composites. The particular aspects of water transport which can be studied by these techniques include a) the kinds of sites occupied by water (voids as well as sites of chemical affinity), b) the uniformity of crosslinks in the epoxy 
matrix, and c) the uniformity of diffusion rates in an epoxy fiberglass composite, i.e. the importance of the epoxy-fiberglass interface in the transport process.

\title{
Accomplishments:
}

The conclusions drawn from the NMR evidence are a) crosslink density is quite uniform on the 3-4 nm scale; b) voids, if present, are very small, containing one or two water molecules (this fits better with the concept of 'free volume' than the concept of 'voids'), and c) the mobility of a typical water molecule, as judged by the linewidth (which reflects a combination of both translational and rotational mobility), increases as the overall amount of water increases, notwithstanding the trend shown by water uptake curves versus time which suggest that diffusion slows down as the amount of water increases. These results suggest that the diffusion processes of water in epoxy and their composites are not complicated by the exclusion of water from certain regions nor the partitioning of water into multiple sites of different energetics. In future work the diffusion constant of water in epoxies, and especially in epoxy-fiberglass composites will be measured and will seek evidence of the inhomogeneous diffusion of water where the inhomogeneity will be linked to the presence of water in interfaces.

This work aims to provide a molecular level description of the distribution and available sites for water in an epoxy. The impact will be information to help in designing epoxies with a greater resistance to water uptake. In particular, the notion that microvoids, whose volumes could contain many water molecules, are not present in any significant amount, eliminates the approach of improving epoxies through more tedious curing procedures. The impact of the planned diffusion measurements is to better understand the transport of water in an epoxy/fiberglass composite. The latter materials are widely used and anything which can be done to improve the performance of composites exposed to water will be helpful. In particular, studies will test the hypothesis that water wicks in along the epoxy/fiberglass interface.

A combination of NMR strategies are employed, including precise chemical shift measurements via magic angle spinning techniques, multiple-pulse proton spin diffusion studies, polarization transfer measurements between water protons and the epoxy protons, and water linewidth measurements as a function of water uptake levels. In the future, a pulsed field gradient facility with high gradient strength will be made operational for performing diffusion measurements on water in epoxies and epoxy composites. The demands of this latter experiment are very high in terms of the intensity and reproducibility of successive field gradient pulses and it remains to be seen if these experiments can be performed properly.

\section{Structural Characterization of Polymers by Small Angle X-ray Scattering}

\author{
J.D. Barnes
}




\section{Objective:}

The objective is to expand the use of small angle x-ray scattering (SAXS) as a technique for characterization of materials and processes through improvements in instrumentation and methods. SAXS is a complementary tool to small angle neutron scattering (SANS) because it utilizes different physical phenomena for obtaining scattering contrast and because it is less subject to certain kinds of interferences. While SAXS is a classical tool for probing microstructure in a wide range of materials it is not easily accessible to users because of the complexities in instrumentation and interpretation, difficulties which could conceivably be ameliorated through increased standardization of instruments and methods.

\section{Accomplishments:}

There is a continuing effort to make the SAXS facility at NIST more accessible to its users through upgrades to the computer systems, the data handling software, and the $\mathrm{x}$-ray camera itself. Users of the facility conduct either collaborative or proprietary research.

Collaborative research activities involve other NIST staff members and researchers from academic institutions, other government agencies, and industrial organizations. Proprietary research is conducted by researchers from corporate laboratories with the assistance of facility staff. General Electric Research Labs, Mobil Chemical Co. R \&D Center, W. R. Grace Co., and Armstrong World Industries have made use of this capability between 1 August 1993 and 1 October 1994.

A new sample carrier system has been made available for use with such heavy sample fixtures as deformation stages, furnaces, or vapor chambers. The original data collection system has been replaced with a desktop personal computer using a graphical interface. New ASCII data file formats produced by the data acquisition process are completely portable among the widest possible variety of computer platforms, greatly facilitating access by the facility's end users.

The data reduction software, which runs on a UNIX workstation, has been completely rewritten to better deal with the statistical nature of the data. Errors arising from "lack of fit" in averaging processes as well as from the Poisson character of the counting process are now properly accounted for. Careful attention has been paid to keeping the software simple, so that it can be compiled and executed on the broadest possible range of computer systems. The "scripting" approach that is natural to UNIX has also been found useful as a documentation device. The data reduction host computer is connected to the Internet, affording maximum convenience to its users.

New "nano-engineered" materials are increasing demands for SAXS data in the intermediate angle range $\left(.02 \AA^{-1} \leq \mathrm{q} \leq 0.5 \AA^{-1}\right)$. These data are critical in assessing the character of interfaces in phase-separated systems including aerogels, block copolymers, composite materials, and semicrystalline polymers. The limiting power law behavior that is accessible to SAXS is also important in gel-forming systems, reactive blends, micellar systems, and processes using in-situ polymerization. An especially effective way of investigating these phenomena for anisotropic scatterers is to display $I\left(q_{x}, q_{y}\right) q^{-p}$ as a two-dimensional image, 
where $I\left(q_{x}, q_{y}\right)$ is the scattering intensity as measured using an area detector, $q$ is the scattering momentum transfer, and $\mathrm{p}$ is a limiting power law exponent.

Most materials scatter weakly in the intermediate angle range. Current attempts to improve capabilities in this area include investigating methods for opening up the pinhole collimation system to increase photon flux at the sample, the use of a shorter scattered beam path to increase the angular range of the camera, and the use of improved area detectors to enhance the signal-to-noise ratio at larger scattering angles.

Materials studies conducted between 1 August 1993 and 1 October 1994 include:

1) Deformation behavior of urethane materials, conducted under an interagency agreement with Dr. Len Buckley, currently at the Naval Research Laboratory. These studies are aimed at elucidating the relationship between microstructure and optical transmission in these materials.

2) Microstructure of sheared block copolymers, a collaborative study between Dr. Catherine L. Jackson of the NIST Blends \& Processing Group and researchers from Michigan Technical University. The work is complementary to SANS studies carried out using an insitu shear cell. The SAXS facility is able to accommodate very tiny samples cut from cone and plate rheometer specimens but SAXS cannot be used for in-situ rheological studies in the way that neutrons can.

3) Interpenetrating polymer networks, IPN's, for printed wiring boards. Dr Barry J. Bauer of the Blends \& Processing Group is the principal investigator in this examination of the limiting power law behavior for systems in which the polymerization of $\mathrm{SiO}_{2}$ competes with polymerization of an acrylic polymer. Studies revealed that the microstructure is very sensitive to polymerization conditions.

4) Cooperative Research with Dow Chemical Co. under the direction of Dr. Gregory McKenna. The SAXS data revealed that certain semicrystalline syndiotactic polystyrene materials exhibit an unusual $\mathrm{q}^{-2}$ limiting power law behavior. Scattering measurements on glassy amorphous materials have demonstrated that the NIST camera is sensitive enough to detect an essentially constant contribution to the scattering that is thought to arise from density fluctuations. These measurements will be extended to specimens undergoing creep to determine whether scattering observations can detect the very earliest stages of craze or crack formation. 


\section{DENTAL AND MEDICAL MATERIALS PROGRAM}

\section{Goals}

The Dental and Medical Materials Program provides measurement methods, data, and concepts needed by the U.S. health care industry to develop dental composite restorative materials of greater durability and wear resistance. Dental restorative composites are heterogeneous materials having three essential phases: 1) a polymeric matrix which comprises the continuous phase, 2) fillers of various types, sizes, shapes and morphologies which constitute the disperse phase and 3) an interfacial phase that, in varying degree, bonds the continuous and disperse phases into a unitary material rather than a simple admixture. The polymeric matrix of a dental composite is formed by free radical polymerization, under ambient conditions, of a resin which is one or more vinyl monomers, usually of the methacrylate class. Polymerization is started either by the formation of initiating radicals from chemical reduction-oxidation( redox) reactions or by photochemical redox reactions. While all three phases are important in determining the properties of the composites, this program focuses primarily on the interfacial and polymer matrix phases. Although only a minor component of these composites, the interfacial phase that develops from the interaction of the silane coupling agent with the polymer matrix and the siliceous filler exerts a profound effect on the properties of the composites. Because these composites are used in an aggressive, aqueous environment that constantly challenges the vulnerable silane mediated polymer-glass bond, understanding of this critical interfacial phase is being acquired so that strategies can be developed for its improvement.

Dental research directions in support of the goals are established in collaboration with the American Dental Association(ADA), the National Institute of Dental Research, and guest scientists from the U.S. Navy and the U.S. Public Health Service. Currently, the ADA Health Foundation sponsors 35 research associates at NIST.

\section{FY-94 Significant Accomplishments}

- Identified a potentially superior silane coupling agent, 10methacryloxydecyltrimethoxysilane, for dental applications.

- Demonstrated that the single-fiber microbond test can be a sensitive, versatile, and facile tool probing the interface that forms between dental polymers and glass surface.

- Demonstrated that the silanization procedure can exert a significant effect on the durability of dental composites.

- Demonstrated that acrylic-vinyl ether monomer systems can be effectively polymerized using dual curing (radical/cationic) initiator systems. 
- Developed a new type of visible light activated photocationic initiator system.

- Developed several new synthetic routes to highly fluorinated monomers and oligomers with well defined structures.

- $\quad$ Synthesized, characterized and polymerized difunctional vinyl spiro orthocarbonate monomers that yielded hard, glassy crosslinked, expanded polymers.

\section{Dental Polymers Designed with Minimal Polymerization Shrinkage, Residual Vinyl Content, Water Sorption and Oxygen Inhibition}

J.W. Stansbury, C. Gingreau ${ }^{1}$, S.I. Kim, G.L. Scott, J.M. Antonucci and J.A. Tesk ${ }^{1}$ Ecole d'Applications des Haute Polymers, Strasbourg, France

\section{Objectives:}

To develop improved polymeric materials for dental resin applications through use of double ring-opening polymerization, novel macromonomers and prepolymers, and

cyclopolymerizable monomers and oligomers to achieve increased degree of conversion along with reduced polymerization shrinkage. Additionally, to increase the hydrophobic nature and resistance to oxygen inhibition of polymerization of dental polymers by use of fluorinated monomers and vinyl ether comonomers.

\section{Accomplishments:}

Synthesis and Characterization of Dental Resin Systems with Reduced Polymerization Shrinkage

The contraction associated with polymerization of resin-based dental composites has complicated the practical use of these versatile restorative materials. Cavity preparation, adhesion-promoting surface treatments and composite placement techniques are all dictated to a large extent by the need to accommodate shrinkage of the resin component. In attempts to minimize or eliminate the curing shrinkage of dental resins, the double ring-opening polymerization of spiro orthocarbonates (SOC) has been suggested as a source of expansion. Difficulties have been encountered with this approach due to the low moduli and limited molecular weights that are characteristic of these ring-opened polymers. The goal of this study was to address the problems of low molecular weight and low modulus, which are characteristic of ring-opened polymers, through the use of bis(SOC) monomers capable of crosslink formation. The key feature of the synthetic route to these difunctional monomers is the use of a hydroxymethyl-functionalized SOC intermediate. Two equivalents of this preformed SOC were coupled through either a diacid chloride or a diisocyanate to provide aromatic bis(SOC) monomers. These monomers, which are liquids at room temperature or low-melting crystallizable solids, were photo-polymerized with a UV-activated cationic initiator to produce glassy, crosslinked polymers with expansion. The combination of conventional methacrylate monomers and bis(SOC) monomers provided free radical/cationic 
dual cure resins which could be photo-cured to give clear polymer films over a broad range of monomer ratios. Alternatively, a true copolymerization of SOC and vinyl ether comonomers was achieved by a cationic mechanism. Ring opening of the SOC component was virtually complete.

A simple method to reduce polymerization shrinkage of an addition polymer is to increase the molecular mass of the monomer relative to the reactive groups. This decreases the number of reactive sites per unit volume in the monomer or resin, and thereby, decreases polymerization shrinkage. Through controlled synthetic procedures, prepolymers with relatively few reactive sites and minimized viscosities can be designed. Beyond the potential reduction in polymerization shrinkage for resins based on these prepolymers, the resulting cured polymers should yield less leachable material and have greater toughness than the highly crosslinked polymers obtained from the polymerization of small dimethacrylate monomers. This macromonomer approach to minimize polymerization shrinkage is a part of the project concerned with design and evaluation of low surface energy fluorinated materials.

Since the discovery that cyclopolymerizable monomers could be produced simply from the amine-catalyzed coupling reaction between acrylates and formaldehyde, developments with this new class of free radical polymerizable monomers have advanced rapidly. Initially, monoacrylates were converted to ether-linked 1,6-diene monomers, termed oxybismethacrylates, which demonstrated a strong propensity toward polymerization via an alternating intermolecular addition/intramolecular cyclization process. It was then shown that high degrees of conversion as well as reductions in polymerization shrinkage (by $30-40 \%$ relative to dimethacrylates after correcting for molecular weight of the monomers and extent of conversion in the polymers) could be obtained due to the cyclization process.

The use of a polymer-supported version of the amine catalyst necessary for synthesis of cyclopolymerizable monomers has shown some interesting applications. Product collected by direct filtration of the swollen catalyst gel had a significantly higher ratio of the cyclopolymerizable 1,6-diene to $\alpha$-hydroxymethylacrylate than did the product subsequently removed by solvent extraction of the filtered gel. These observations indicate a preference for the more polar $\alpha$-hydroxymethylacrylate product to be located within the network of the polymeric catalyst. Therefore, there is the potential to make modifications within the reactants and/or the polymeric support structure that would further emphasize this bias and simplify product purification. Use of a polymeric catalyst would also greatly facilitate catalyst removal in the preparation of water soluble cyclopolymerizable monomers and oligomers as well as in the single-step synthesis/formulation technique. Addition of a solvent, such as DMSO, to the reaction involving the polymeric catalyst resulted in a substantial increase in rate.

Low Surface Energy Monomers with Varied Fluorine Contents and Distributions.

Fluoropolymers, noted for outstanding durability in harsh environments, have not been utilized in dental materials despite their extreme hydrophobicity and chemical resistance. 
Prior studies of fluorinated resin-based composites revealed mechanical property deficiencies which limit utility of these unique materials. This study was devised to better define the influence of fluorine content $(\% \mathrm{~F})$ and its structural distribution on properties of fluorinated resins and their composites. A series of dimethacrylate monomers was prepared by reaction of fluorine-containing aromatic diepoxides with fluoroalcohols followed by conversion of the resulting diols to the dimethacrylates. The viscosity of each of the monomeres, as well as that of various mixtures with a comonomer, was dramatically reduced compared with conventional Bis-GMA resins by introducing fluorine as compact trifluoromethyl $\left(\mathrm{CF}_{3}\right)$ backbone segments or as long $\left(\mathrm{C}_{8}-\mathrm{C}_{9}\right)$ fluoroalkyl pendant chains. For the series of monomers synthesized, \% F increased from 23 to $60 \%$, molecular weight increased from 993 to 1941 , and refractive index decreased from 1.500 to 1.363 . Water contact angle measurements directly correlated with the overall fluorine content. Preliminary evaluation of photocured, silanized quartz-filled composites based on these fluoromonomers with the comonomer, 1,10-decamethylene dimethacrylate, provided diametral tensile strength (DTS) values ranging from $3.3 \pm 0.4 \mathrm{MPa}$ for the monomer with the maximum $\% \mathrm{~F}$ (and fluorine incorporated primarily as long chains) to $37.3 \pm 1.7 \mathrm{MPa}$ for a moderately fluorinated monomer (with $\mathrm{CF}_{3}$ groups as the only fluorine source). The resin with the highest fluorine content underwent phase separation during polymerization with the resulting increase in opacity severely limiting the degree of conversion and strength of the polymer.

A related synthetic approach gave fluorinated urethane methacrylate oligomers in which mixtures of $\mathrm{CF}_{3}$ and long fluoroalkyl substituents were combined to maximize strength and minimize viscosity. A composite based on such an oligomer had a DTS of $49.7 \pm 1.5 \mathrm{MPa}$ with a water uptake of only $0.2 \%$. These results indicate that both fluorine content and its distribution are important parameters in designing fluorinated resins for dental applications. It is expected that appropriately designed fluorinated resins will yield composites with excellent mechanical strength and resistance to oral fluids. With the relatively low densities of polymerizable groups and the low viscosities, which minimize the need for diluent comonomers, resins based on these fluorinated monomers and oligomers should polymerize with low shrinkage.

Novel highly-fluorinated cyclopolymerizable monomers and oligomers were also prepared through an amine-catalyzed reaction using fluorine-containing acrylates or diacrylates with paraformaldehyde. Appropriate fluorine placement allows efficient formation of the 1,6diene ether linkage necessary for effective cyclopolymerization. Free radical solution polymerization of a 1,6-diene monomer $(56 \% \mathrm{~F})$ resulted in precipitation of a completely cyclized polymer which was soluble only in Freon-type solvents. In an alternate approach, the formaldehyde/amine reaction was applied to fluorinated diacrylates to provide cyclopolymerizable oligomers ( 40 to $50 \% \mathrm{~F}$ ) with multiple 1,6-diene linkages situated along the prepolymer backbones. Introduction of fluorine reduces the viscosity of the oligomers relative to hydrocarbon analogs. Polymerization of these fluorinated oligomers provides crosslinked fluoropolymers which may be useful as low surface energy coatings or as stable matrix materials. 
Visible Light Polymerization of Vinyl Ether-Acrylic Monomer Systems.

Oxygen inhibition of the free radical polymerization of methacrylate monomers is the result of an interaction of a radical species with molecular oxygen to yield a hydroperoxide which is generally not reactive to further decomposition under near-ambient polymerization conditions. When dispensed for use in a restoration, oxygen begins to diffuse into the resin and upon irradiation, the relatively high concentration of oxygen at the resin/air interface results in rapid quenching of a significant portion of the radical sites present. Near the resin surface, the oxygen concentration remains sufficiently high to effectively halt the polymerization at low degrees of conversion. The end result is generally a polymer that is well cured in the bulk portion but surrounded on the exposed surfaces by a poorly cured layer that ranges between a tacky solid and a viscous liquid. This results in difficulties when thin layers of resin must be polymerized without substantial post-cure surface polishing, as in veneer, sealant and adhesive applications. The ability to apply and efficiently polymerize thin layers of protective resins to the entire exposed surface of a tooth, rather than to just the pit and fissure pockets, may provide a means of enhancing the scope of preventative dentistry.

Because of their many excellent properties (facile polymerization, optical clarity, acceptable mechanical strength), acrylic monomers and polymers have long played a dominant role in dentistry. However, acrylic based dental materials have several critical shortcomings including susceptibility to oxygen inhibition which limits their conversion and physicochemical stability in the oral environment. These limitations influence how resinbased polymers are employed in dentistry and in some applications, eliminate them from consideration. The recent development of photocationic initiator systems now makes it feasible to consider non-acrylic monomers such as vinyl ethers for use in polymeric dental materials. Vinyl ethers are noted both for their ability to undergo rapid cationic polymerization (free of oxygen inhibition) and free radical copolymerization. The goals of this study were to develop visible light activated initiator systems with capability for cationic/free radical polymerization and apply these to explore the feasibility of polymerizing vinyl ether-acrylic monomer systems.

Analysis of photocured unfilled resin films by FTIR indicated that while pure vinyl ether monomers could be converted to polymers with no measurable residual unsaturation, resins with vinyl ether and Bis-GMA comonomers showed high methacrylate and low vinyl ether conversions. A systematic study into the cause of the reduced activity of the vinyl ether has identified the methacrylate comonomer, rather than the camphorquinone or aromatic amine employed in the dual cationic/free radical photoinitiator system, as the predominant negative factor limiting the extent of vinyl ether polymerization. Preliminary results have shown that by increasing the acyl phosphonate sensitizer concentration, high conversion of both monomer types is possible without the use of the CQ/amine free radical initiator system. Using a simple visible light activated photoinitiator system comprising an iodonium salt plus an acyl phosphonate, well cured polymers based on hybrid vinyl ether/acrylic monomer systems are possible. These novel monomer and initiator systems have potential for use in 
dental composites, sealants and adhesives.

\section{Dental Composites with Improved Interfaces}

J.M. Antonucci, J.A. Tesk, W.G. McDonough, C.L. Schutte, S.I. Kim, J.R. Kelly ${ }^{1}$, J.W. Stansbury, H. Kikuchi ${ }^{2}$ and M. Nishiyama ${ }^{2}$

${ }^{1}$ U.S. Navy Dental School, Bethesda, MD

${ }^{2}$ Nihon University, Tokyo, Japan

\section{Objectives:}

To develop improved silane coupling agents and silanization procedures for enhancing the interfacial phase of dental composites and related materials. An ancillary goal is to compare the sensitivity of several mechanical tests as a means of assessing the quality of the interfacial phase.

\section{Accomplishments:}

Resin based composites, originally developed as esthetic filling materials for anterior teeth and for other low stress dental applications, are increasingly being used in high stress bearing situations, e.g. inlays, onlays, posterior restoratives. In order to better assess the capabilities of the rapidly growing number of newly improved composites for these and other uses, it is necessary to understand their nature and to have tests that will accurately characterize their strength and overall durability, at least in vitro.

Polymeric dental composites are interconnected heterogeneous materials that generally have three discernable phases: (1) a polymeric matrix or continuous phase formed by

polymerization of a resin system, (2) a higher modulus dispersed phase consisting of fillers of various types, sizes, shapes and morphologies and (3) an interfacial phase that bonds to both the continuous and dispersed phases thereby forming a unitary material. In these resin based dental composites adhesion between the polymeric matrix and the reinforcing filler is usually achieved by use of silane coupling agents, e.g. 3-methacryloxypropyltrimethoxysilane (MPTMS). MPTMS, is currently used in dental composites and related acrylic dental materials. The degree of reaction of the silane with the glass filler (oxane bond formation) and with the resin system (by copolymerization) determines the efficacy of a coupling agent. The oxane bond that forms between the silane agent and the mineral filler is especially vulnerable to hydrolysis. The physical-chemical nature of the silane agent (e.g. size, degree of hydrophobicity, reactivity, number of reactive groups, modulus, polymerization shrinkage), the silanization procedure and extent of filler coverage are important parameters that will determine the durability of the interface/interphase. In addition, the nature of the glass filler also can affect the durability of the interfacial silane phase. Thus certain radiopaque glasses are vulnerable to hydrolytic degradation reactions which can weaken bonding at the glass interface.

In order to determine the quality and durability of the interfacial phase in composites and 
related materials, it is necessary to employ tests that can accurately characterize the interfacial strength that exists between the matrix and filler and how the interfacial phase responds to environmental challenges. The biaxial flexure test and two types of single-fiber methods (the microbond bead and single-fiber fragmentation tests) hold promise for providing new insights into mechanisms of interfacial adhesion and degradation. Surface analysis of fractured interfaces by FT-IR and SEM spectroscopy and similar techniques also can aid in elucidating interfacial failure mechanisms.

While modest water absorption by polymeric dental composites can have some beneficial effects (e.g. partial compensation for polymerization shrinkage, release of internal strains), excessive water uptake produces a highly plasticized and weakened polymeric phase (matrix plus interfacial phases). Chemical degradation of the matrix and interfacial phases can follow resulting in exposure of the filler phase, leading to composites with deficient mechanical and physical properties. For example, excessive water absorption acts to decrease the strength and elastic moduli of composites, while enhancing their creep under compression. Degradation of the interfacial phase by hydrolytic reactions at the silane-glass interface, in turn, exposes the mineral fillers to degradative hydrolytic reactions, especially radiopaque fillers that contain certain types of metal cations, e.g. barium, strontium, zinc. To stabilize the interfacial phase against hydrolytic attack, silane agents are needed that copolymerize well with the resin, form optimal bonds with the mineral phase and that form chemical structures that are flexible, strong and water resistant.

\section{Silane coupling Agents: Comparison of MDTMS versus MPTMS}

A study was initiated to compare the efficacy of 10-methacryloxydecyltrimethoxysilane (MDTMS) and 3-methacryloxypropyltrimethoxy-silane (MPTMS) as silane coupling agents for polymer based dental composites. From its structure, MDTMS is expected to provide greater flexibility and hydrophobicity at glass filler-polymer interfaces than the smaller MPTMS. A macrosized, radiopaque particulate flass filler (Corning Glass 7724) was silanized using equivalent amounts of MPTMS and MDTMS according to previously described procedures involving either cyclohexane (hydrophobic) or aqueous methanol (hydrophilic) as the silanization solvent. The MPTMS and MDTMS-silanized glasses were used to prepare composite pastes with a resin consisting of Bis-GMA/TEGDMA (1/1, w/w) that was activated for visible light polymerization with $0.2 \%(\mathrm{w} / \mathrm{w})$ camphorquinone $(\mathrm{CQ})$ and $0.8 \%(w / w)$ ethyl 4-dimethylaminobenzoate (4EDMAB).

Both the diametral tensile strength (DTS) and the flexural strength (FS) values of the MDTMS based composites were comparable or better than MPTMS based composites at the same powder-liquid ratio. A noteworthy property of MDTSM compared to MPTMS is that significantly higher filler loadings can be easily achieved with the longer silane agent. Preliminary results also indicate that MDTMS may be offering greater protection of composite interfaces from the effects of water exposure.

Single-Fiber Tests for Evaluation of Interfacial Properties: Interfacial shear strength 
measurements by the microbond test

Traditionally fracture tests (e.g., diametral tensile, uniaxial tensile, flexural strength) have been used to study interfaces in polymer based materials, e.g., dental composites. However, interpretation of interfacial failure mechanisms from strength values derived from bulk property tests can be difficult because of the interaction of many competing failure modes that accompany composite fracture. Single fiber tests such as the microbond test (MBT) and the single-fiber fragmentation test (SFFT), have the potential to reduce the complexity of events that confound composite interfacial failure analysis and allow better identification of factors that control interfacial adhesion between dental polymers and glass surfaces.

The microbond test involves putting drops of resin onto a fiber, allowing surface tension to form a symmetrical drop, and then curing the resin. The fiber with the cured drops on it is attached to a tab, which in turn is attached to a load sensing device. After this, the blades of a blade micrometer are brought into contact with the upper surface of the drop. The blades, controlled by an actuator, impart a load on the drop until the blades shear the drop off the fiber. The interfacial shear strength is calculated by dividing the load needed to debond the drop by the bonded area.

In this study, MBT was investigated as a method to probe the interaction of methacrylate polymers with glass surfaces. A $2 \times 3$ factorial design was used in this study. The factors investigated were unsilanized and silanized E-glass fibers with the 3 visible light activated resins: A) Bis-GMA/TEGDMA (7/3 w/w); B) UDMA, a urethane dimethacrylate derived from HEMA and 2,4,4-trimethylhexane-1,6-diisocyanate, TMHDI; and C) FUDMA, a flexible poly(ethylene glycol) urethane dimethacrylate derived from TMHDI, 2-hydroxyethyl methacrylate and a polyethyleneglycol (PEG 200). The resins were applied as symmetrical droplets to unsilanized and silanized single glass fibers, photocured for 3 minutes under visible light irradiation and stored at $23^{\circ} \mathrm{C}$ in air for $24 \mathrm{~h}$. Mean shear bond strengths (SBS) in $\mathrm{MPa}, \mathrm{n} \geq 7$, obtained with the MBT apparatus were: $\mathrm{A})$ unsilanized: 10.0 (1.5), A) silanized: 29.1(2.8); B) unsilanized: 17.0(2.9), B) silanized: 36.5(5.9); C) unsilanized: 6.1(1.5), C) silanized: 18.8(4.2). Resin B gave the highest SBS for both unsilanized and silanized glass fibers and demonstrated about a twofold increase for silanized vs. unsilanized fibers; A and $\mathrm{C}$ showed about a threefold increase in bond strength for unsilanized vs. silanized fibers. $C$, the most flexible resin, exhibited the lowest SBS with both unsilanized and silanized fibers. Two-way ANOVA showed that both the resin and glass as well as their interaction were highly significant $(\mathrm{P}=0.0001)$. Photomicrographs of the polymer-fiber specimens indicated that the failure occurred at the interface. These results indicate that the MBT is a promising, facile method for assessing the quality of the interface that forms between dental polymers and glass surfaces. A complementary study is in progress to compare different silane coupling agents, e.g. MDTMS versus MPTMS.

Interfacial Shear Strength Measurements by the Single-Fiber Fragmentation Test

In addition, the single-fiber fragmentation test (SFFT) also is under evaluation as a method to 
study the interface in dental composites. The SFFT involves securing a fiber into a dogboneshaped cavity of a silicone mold, then pouring the resin into the cavity. Visible-light irradiation is used to cure the resin. The sample is removed from the mold, placed into a loading jig and loaded in tension to induce breaks in the fiber. The SFFT essentially applies a tensile load to generate a interfacial shear stress between the fiber and resin. If the matrix is transparent, visual observation of the failure process is possible. Unlike the MBT, the matrix of SFFT samples must have a higher strain to failure value than that of the fiber. The tensile force is increased until no further breaks occur. At this point, the sample has reached saturation. Statistical analysis is conducted on the distribution of fiber lengths, and this information is then combined with information of the tensile strength of the fiber and the fiber diameter to estimate the interfacial shear strength of the fiber/matrix bond. Preliminary tests indicate that the Bis-GMA resin A yields too brittle a polymer to be useful in this test. In addition to resins $B$ and $C$, other monomer systems will be investigated for their applicability with the SFFT.

\section{Effect of Silanization Methods on Composite Strength and Durability}

An important factor in determining the strength and durability of polymer matrix composites is the method of silanization employed to modify the filler surface. The chemistries by which the silane agent attaches itself to the glass filler can be altered by changes in the solvent, catalyst, temperature and post treatments employed in the silanization procedure. A study was initiated to determine the effect of four silanization methods on the strength and durability of dental composites formulated with a visible light activated Bis-GMA/TEGDMA $(1 / 1 \mathrm{w} / \mathrm{w})$ resin and a macrosized $(5.5 \mu \mathrm{m})$ silica filler treated with $0.7 \mathrm{wt} \%$ MPTMS. The following silanization methods were employed: A:MPTMS in methanol/water $(95 / 5 \mathrm{w} / \mathrm{w})$; stirred $2 \mathrm{~h}$ at room temperature; silica filler added and the mixture heated to remove the solvent; the dried filler was then heated for $2 \mathrm{~h}$ at $110^{\circ} \mathrm{C}$. A'; same as A, but the silanized filler was washed with dichloromethane to remove loosely attached silane moieties. B: MPTMS was dissolved in cyclohexane containing $2 \mathrm{wt} \% \mathrm{n}$-propylamine (based on the weight of filler), silica filler was added and stirred with heating to remove the solvent; the dried silanized silica filler was then heated for $2 \mathrm{~h}$ at $110^{\circ} \mathrm{C}$. $\mathrm{B}^{\prime}$ : same as $\mathrm{B}$, but the post treatment of washing with dichloromethane was included. These silanized fillers were then immersed separately in distilled water at $60^{\circ} \mathrm{C}$ for 1 or 2 weeks. After filtration and desication, fillers $A, A^{\prime}, B$, and $B^{\prime}$ were used to prepare visible light cured composites containing $76 \mathrm{wt} \%$ of these glass fillers. The efficacy of the various silanization methods was assessed using various mechanical tests (uniaxial tensile, compressive and flexural strength tests).

Composites formulated with fillers silanized by Methods $\mathrm{A}$ and $\mathrm{A}^{\prime}$ and conditioned in $60^{\circ} \mathrm{C}$ water 1 and 2 weeks, respectively, showed significant deterioration in mechanical properties compared to their controls (composites formulated with unconditioned silanized fillers, A and $A^{\prime}$ ). Composites based on silanized fillers prepared by Methods B and $B^{\prime}$ which has been immersed in $60^{\circ} \mathrm{C}$ water for 1 week exhibited excellent retention of mechanical strength compared to their controls. However, similar composites based on the use of these silanized 
fillers, when conditioned for 2 weeks in $60^{\circ} \mathrm{C}$ water, showed significant differences in mechanical properties. Composites with fillers silanized by Method B' (dichloromethane wash) showed significant reductions in their uniaxial tensile, compressive and transverse strengths. These results were comparable to the results obtained with composites based on similarly conditioned fillers that were silanized by Methods A and A'. Significantly, composites formulated with fillers silanized by Method B, even after 2 weeks immersion in $60^{\circ} \mathrm{C}$ water, exhibited excellent retention of mechanical strength.

A possible explanation for the observed differences in the durability of the silane coating on these silica fillers lies in the different chemistries of the silane coupling agent MPTMS in methanol/water and in cyclohexane with n-propylamine as a catalyst. Analysis of the dichloromethane washings of silanized fillers prepared by Methods $A^{\prime}$ and $B^{\prime}$ by gel permeation chromatography revealed significant differences in their compositions. Washings from Method $\mathrm{A}^{\prime}$ contained predominantly trimers and tetramer molecular species derived from MPTMS, whereas those from Method $\mathrm{B}^{\prime}$ consisted mainly of a dimer product. In methanol/water systems, MPTMS exists in a hydrolyzed form which can undergo condensation reactions via the silanol groups to form dimers, trimers, tetramers, etc. prior to being absorbed on silica. By contrast, in cyclohexane (Method B) a minimal amount of water is present, mainly from water adsorbed on the silica powder, and the reaction conditions probably favor adsorption of mainly the monomeric silanol form of MPTMS on silica. Thus, different silanization conditions (solvent, acidity, basicity, temperature, etc.) may generate different molecular species from the silane agent which, in turn, may exhibit different adsorption behaviors on silica. In addition, the deposition of most silane coupling agents occurs by a complex adsorption mechanism and usually results in multilayer surface coatings, consisting of both chemisorbed and physisorbed components, rather than monolayers. The overall results of this study indicate that silanization of silica fillers in hydrophobic solvents such as cyclohexane with a basic amine catalyst can lead to silane coatings with enhanced environmental durability. However, removal of physisorbed silane components present on this type of silanized silica appears to have an adverse effect on the durability of the silane coating.

\section{Improved Adhesive Systems for Bonding to Dentin}

J.M. Antonucci, G.E. Schumacher ${ }^{1}$, J.E. Code $^{1}$ and P.S. Bennett ${ }^{2}$

${ }^{1}$ U.S. Public Health Service

${ }^{2} \mathrm{ADAHF} / \mathrm{PRC}$

\section{Objectives:}

To obtain better understanding of the mechanisms of bonding to dentin so that more efficacious bonding systems can be developed.

\section{Accomplishments:}


Dental adhesives often employ acidic etchants to cleanse tooth surfaces. These aggressive surface treatments add extra steps to the bonding protocol and can cause damage to the tooth substrate, leading to irratation or even injury. $N$-phenyliminodiacetic acid (PIDAA) is a unique amino acid of moderate acidity $\left(\mathrm{pK}_{1}=2.4\right)$ with excellent chelating ability and the ability to initiate or coinitiate the free radical polymerization of a variety of dental monomers. In contrast to $\mathrm{N}$-phenylglycine (NPG) and similar aryl amino acids, which in aqueous solvents do not remove the smear layer from dentin, PIDAA in aqueous solvents such as aqueous acetone effectively removes the smear layer from dentin surfaces without excessive opening of dentinal tubules, and with minimal damage to the underlying sound dentin and pulpal tissues. Etching with PIDAA does not require an aqueous wash or air blowing step as is the case with strong acid etchants. In addition, scanning electron microscopy of PIDAA-treated dentin also reveals that these tubules are obturated with debris. The obturation of dentinal tubules acts as a seal against harmful chemicals as well as a barrier to the flow of dentinal fluids that can adversely affect the durability of dentin bonding. Treatment of PIDAA-infused dentin with dental adhesive monomers, especially carboxylic acid monomers, results in efficient interfacial polymerization that translates into strong bonding of composites to dentin. Consistently high bond strengths have been routinely obtained using PIDAA. These results demonstrate that PIDAA as a single agent can function as an etchant, a primer, a polymerization initiator or coinitiator, and an obturator. Its use provides a simple, minimally tissue-damaging, two-step procedure for effective bonding to dentin.

\title{
Bioactive Polymeric Dental Materials Based on Amorphous Calcium Phosphate with Remineralization Potential
}

\author{
J.M.Antonucci, D.Skrtic ${ }^{1}$ and E.D.Eanes ${ }^{2}$ \\ ${ }^{1}$ Ruder Boskovic Institute, Yugoslavia \\ ${ }^{2}$ National Institute of Dental Research (NIDR), NIST
}

\section{Objectives:}

To develop polymer matrix materials with remineralizing potential.

Because of their excellent biocompatibility with both soft and hard tissue, crystalline calcium orthophosphates, especially hydroxyapatite (HAP) and those capable of converting to HAP, are finding increasing use as prophylactic, prosthetic, adhesive and restorative materials in dental and other biomedical applications.

By contrast, amorphous calcium phosphate (ACP), which has an apparent compositional formula of $\mathrm{Ca}_{3}\left(\mathrm{HPO}_{4}\right)_{0.2}\left(\mathrm{PO}_{4}\right)_{1.87}\left(\mathrm{H}_{2} \mathrm{O}\right)_{x}$ ( $\mathrm{x}$ is approximately 3 ) and is an important intermediate in HAP formation, has received far less attention as a biomaterial. The two principal reasons for this are ACP's high solubility in aqueous media and its rapid conversion to HAP. These very properties, however, suggest its use as a bioactive filler in polymeric materials, e.g. composites, sealants, adhesives, that can preserve teeth against demineralization and possibly promote remineralization of defective tooth structure. Visible 
light curable composites based on several types of acrylic monomer systems and a stabilized ACP were prepared, polymerized and evaluated for their ability to release calcium and phosphate ions in the presence of an aqueous environment in a sustained fashion and at levels adequate to favor HAP formation.

The resins for these composites were formulated by mixing typical dental commercial monomers in the proportions indicated in Table 1: 2,2-bis[2'-hydroxy-3'methacryloxypropoxy)phenyl]propane (BisGMA), triethylene glycol dimethacrylate (TEGDMA), 2-hydroxyethyl methacrylate (HEMA), 3-methacryloxypropyltrimethoxy silane ((MPTMS) and zirconyl dimethacrylate (ZrM). Camphorquinone (CQ) and ethyl-4-N,Ndimethylamino benzoate (4EDMAB) were used as the photooxidant and photoreductant components, respectively, of the visible light photoinitiator system.

Table 1.

\begin{tabular}{|c|c|c|c|c|c|c|c||}
\hline \multicolumn{7}{|c|}{ Composition of Resins Used for Preparation of Composite Disk Specimens } \\
\hline \hline \multirow{2}{*}{$\begin{array}{c}\text { Resin } \\
\text { system }\end{array}$} & \multicolumn{7}{|c||}{ Resin composition (wt\%) } \\
\cline { 2 - 9 } & Bis-GMA & TEGDMA & HEMA & ZrM & MPTMS & CQ & 4EDMAB \\
\hline \hline 1 & 49.5 & 49.5 & - & - & - & 0.2 & 0.8 \\
\hline 2 & 35.1 & 35.1 & 28.0 & 0.8 & - & 0.2 & 0.8 \\
\hline 3 & 44.5 & 44.5 & - & - & 10.0 & 0.2 & 0.8 \\
\hline
\end{tabular}

Composite pastes were prepared using $40 \mathrm{wt} \% \mathrm{ACP}$ as the particulate filler and $60 \mathrm{wt} \%$ of each of the monomer systems shown in Table 1 as the resin component. Disk specimens were prepared by inserting the paste into circular teflon molds, covering the open ends with mylar lined glass slides, and irradiating each side for $120 \mathrm{~s}$ with blue light $(\sim 470 \mathrm{~nm})$. The specimens were postcured in their mold assemblies at $37^{\circ} \mathrm{C}$ for $18 \mathrm{~h}$ and, after removal from their molds, the composite disks were weighed and examined by $\mathrm{x}$-ray diffraction (XRD). To prevent excessive conversion of ACP to HAP within the composite structure, the ACP powder used in these composites was stabilized by the incorporation of pyrophosphate ions $\left(\mathrm{P}_{2} \mathrm{O}_{7}{ }^{4}\right)$ during its preparation. A portion of this $\mathrm{P}_{2} \mathrm{O}_{7}{ }^{4}-\mathrm{ACP}$ also was silanized with MPTMS in order to assess the influence of the silane coating on the release of calcium and phosphate ions.

Each composite disk was immersed in $100 \mathrm{ml}$ of continuously stirred, HEPES buffered $(\mathrm{pH}=7.4)$ sodium chloride solution $(240 \mathrm{mOsm})$ at $37^{\circ} \mathrm{C}$. The release of calcium $\left(\mathrm{Ca}^{2+}\right)$ and total phosphate $\left(\mathrm{H}_{2} \mathrm{PO}_{4}{ }^{1-}, \mathrm{HPO}_{4}{ }^{2-}, \mathrm{PO}_{4}{ }^{3-}\right)$ ions $\left(\mathrm{PO}_{4}\right)$ from the disks was kinetically monitored for up to $500 \mathrm{~h}$ using atomic absorption spectroscopy $(\mathrm{Ca})$ and $\mathrm{UV}$ spectrophotometry $\left(\mathrm{PO}_{4}\right)$. Upon completion of the immersion tests, the disks were removed, air dried, and reweighed and characterized by XRD. 
The results of this study were as follows: composites based on the relatively hydrophobic BisGMA/TEGDMA monomer system (resin 1) and unsilanized ACP, showed the lowest $\mathrm{Ca}^{2+}$ and $\mathrm{PO}_{4}$ release rate. By contrast, composites which were formulated with ACP that had been presilanized with MPTMS and resin 1, exhibited a significantly higher release rate of $\mathrm{Ca}^{2+}$ and $\mathrm{PO}_{4}$ ions. Possibly, the enhanced ion release profile in this composite may be the result of an enhanced degree of homogeneous dispersion of the filler into the polymeric matrix that can be achieved with silanized ACP compared to untreated ACP. Preliminary studies also indicate that a similar improved dispersion of ACP into resin, with accompanying high $\mathrm{Ca}^{2+}$ and $\mathrm{PO}_{4}$ ion release, can also be achieved when MPTMS is added as a additional comonomer to the BisGMA/TEGDMA system (resin 3, Table 1) before mixing with unsilanized ACP to form the composite. Resin 2, which contained the hydrophilic HEMA as one of its monomeric components, yielded an ACP composite with the highest release rate of $\mathrm{Ca}^{2+}$ and $\mathrm{PO}_{4}$. XRD analysis showed that the amorphous nature of presilanized ACP is similar to that of untreated ACP and is maintained in this state in the composite formulations before immersion in the aqueous media.

On immersion in buffered saline, conversion of modest amounts of ACP to HAP occurred internally in these composites. Because of the preponderance of unconverted ACP in these composites, they are able to sustain release of $\mathrm{Ca}^{2+}$ and $\mathrm{PO}_{4}$ ions over long periods of time. Solutions supersaturated with respect to $\mathrm{Ca}^{2+}$ and $\mathrm{PO}_{4}$ ions obtained from this and the other composites were above the minimum needed for remineralization to occur. Mineralization, however, did not occur spontaneously in these experiments, but required the introduction of exogenous HAP seed crystals. Recent experiments using an ACP/polymeric sealant with bovine teeth having artificial caries lesions showed significant remineralization via HAP formation.

\section{Support for the Biomaterials Integrated Products Industries}

J.A. Tesk, J.R Kelly ${ }^{1}$ and G.S. Fishman ${ }^{2}$

${ }^{1}$ U.S. Navy Dental School, Bethesda, MD

${ }^{2}$ Alfred University, Alfred, NY

\section{Objective}

The objective is to support U.S. industries that manufacture biomaterial integrated products.

Efforts aimed at supporting the industries that manufacture biomaterial integrated products involved planning for future initiatives, workshops to identify needs and participation on standards related boards and committees.

\section{Accomplishments:}

Major contributions were made to two international standards, ISO6871-1 and 6871-2, that avoided putting at risk the foreign sales of dental alloys of economic importance to several American dental companies. 
A recent effort has been aimed at exploring the needs of the biomaterials-integrated products industry and how expertise from existing NIST programs may be applied toward meeting those needs. A workshop was held on September 1-2, 1994, with orthopedic manufacturers and the Health Industry Manufacturers Association, to address needs of the orthopedic industry. A priority list was identified by representatives from seven companies, the NIST Polymers and Ceramics Divisions, the Food and Drug Administration, the Mayo Clinic and Alfred University. The compatibility of implant materials with body tissues and effects of wear are leading concerns. Prior to clinical use new materials are currently evaluated by expensive and time-consuming in vitro joint-simulation wear studies. Replacement of jointsimulation tests by simplified, inexpensive and reliable technique(s) is a high priority. New test procedure(s) would be used to screen materials and could accelerate the gathering of data for identifying materials interactions in wear processes. Other needs discussed included: the potential usefulness of polyethylene particulate standards for studies of the contribution of wear debris toward osteolysis (the dissolution of bony tissue) leading to loosening of artificial joints in about $5-10 \%$ of patients over a 10-15 year period, Characterization of materials as affected by processing conditions, and the development of a synthetic synovial fluid to replace bovine serum currently used in wear studies. Follow-up workshops are planned for the development of a NIST-industry program to address these issues and those of other industry segments, such as cardiovascular, bone replacement, and other biomaterial product manufacturers.

\section{Durability and Processing of Dental Ceramic, Metallic and Ceramic-Metal Materials}

J.A. Tesk, J.R. Kelly ${ }^{1}$, K. Asaoka ${ }^{2}$, O. Okuno ${ }^{3}$, J. Lechner ${ }^{4}$, S.C. Saunders ${ }^{5}$, R. Sorensen ${ }^{6}$, H. Morris ${ }^{7}, \&$ R. Mais ${ }^{8}$

${ }^{1}$ U.S. Navy Dental School, Bethesda, MD

${ }^{2}$ Tokushima University, Tokushima, Japan

${ }^{3}$ Tohoku University, Sendai, Japan

${ }^{4}$ Statistical Engineering Division, NIST

${ }^{5}$ Washington State University, Pullman, WA

${ }^{6}$ University of California, Los Angeles, CA

${ }^{7}$ Department of Veterans Affairs, Ann Arbor, MI

${ }^{8}$ Department of Veteran Affairs, Hines Hospital, Hines, IL

\section{Objective}

The objective is to provide analytical and processing information that can be useful in the choice of materials for improved dental prostheses.

\section{Accomplishments:}

A collaborative effort was undertaken with the Department of Veterans Affairs, Washington State University and the NIST Statistical Engineering Division to analyze clinical data taken from a large study of the durability of dental ceramic-metal prostheses. The objective was to examine predictions of survivability using a two-parameter Weibull model for data that included left-, right-, and interval censoring and how such analysis may be useful in 
prospective design of clinical experiments by changing the censoring conditions. The analysis of the clinical data developed Weibull parameters that produce a survival curve that is consistent with experience up to the right-hand censoring time (ca. 10 years) and predicts a $10 \%$ survival of $30 \pm 10$ years ( \pm values are for one standard deviation). An extension of the investigation is planned to explore the sensitivity of confidence intervals to various censoring parameters (patient recall times and intervals).

Ceramic-on-ceramic and metal-on-metal prostheses offer durability (esp. ceramic-on-metal) and esthetics (esp ceramic-on-ceramic). However, there are significant failures with each system. Improvements in performance require material/system properties as affected by processing variables and a better understanding of the sources for failure of prostheses. Significant progress has been made on a number of fronts by U.S. Navy and NIST work. A mixed-hazard Weibull failure model that employs finite-element stress analysis has been able to reproduce the essential features of in vitro and in vivo failure probability and failure origin of a high-strength ceramic core prosthesis with a porcelain veneer. Parametric studies can be conducted with the model to examine how changes in survival may be affected by prosthetic design (including veneer-core thicknesses) and properties. Other work on test methods led to related results that implicated the interface as an important source of failure in both ceramicon ceramic and ceramic-on-metal prostheses.

As mechanical properties and residual stresses from processing affect survivability, collaborative work with Tokushima University applied the indentation-microfracture method to learn how applicable this method may be for determination of fracture toughness and residual stresses in porcelain systems. Two dental porcelain systems were chosen for their extremes in crystallinity and subjected to variations in processing ranging from slow cooling to forced-air quenching. One system displayed marked sensitivity of crystallinity to processing and, therefore, was found unsuitable for direct use of the indentation method for determination of residual stresses (a consistent microstructure is needed to apply this method). As a result, it was concluded that caution needs to be employed in the routine application of this method to determine residual stresses as affected by processing of dental porcelain systems. 


\section{THEORY AND MODELING PROGRAM}

\section{Theory and Modeling of the Transport Properties of Polymer Solutions}

\section{J. F. Douglas}

\section{Objective:}

The processing of polymer fluids often involves mixing particulate matter, small molecule solvents and different types of polymers ("blends") to create complex mixtures. An understanding of the transport properties of such materials is very important for the control of fabrication processes and the ultimate material properties of these composite products.

\section{Accomplishments:}

A novel method is introduced for calculating the friction coefficient of polymer chains and other complex-shaped objects. The method has proven to give accurate results where exact or numerically accurate results are known. The problem of determining polymer friction from molecular parameters (size and shape of monomer, molecular weight of polymer, ...) was critically reviewed to assess the practical limitations of current theories of polymer solution hydrodynamics. The theory has recently been extended to describe transport properties (viscosity, thermal and electrical conductivity, dielectric constant,...) of immiscible polymer blends.

The cooperative diffusion coefficient of a suspension of fluid or solid droplets is found to be related to the viscosity and osmotic compressibility of the suspension. The theory also applies to a composite of polymer and solid filler and to blends of immiscible polymers.

A model of diffusion-limited polymer desorption kinetics was introduced which leads to a stretched exponential decay of the adsorbed polymer concentration and a characteristic time for the desorption process which varies inversely to the polymer solution diffusion coefficient. The predictions of the model were confirmed in experimental studies by Steve Granick and coworkers at the University of Illinois. Similar stretched exponential kinetics has recently been observed at NIST in the mixing of spin-cast homopolymer layers.

\section{Theory and Modeling in Polymer Physics}

\section{E.A. DiMarzio, P.H. Verdier and S.C. Glotzer}

\section{Objectives:}

The scientific underpinnings of the phase behavior of multiphase polymers are just beginning to approach full development. Implementation of current theory into computer simulations for study of the dynamics of phase separation and the resulting morphologies are important undertakings. Furthermore, the kinetics of the glass transition event, in spite of significant 
effort over the years, are relatively poorly understood on a microstructural level. Theory and modeling are needed to make further progress and to establish connections to the mechanical response of engineering polymers. The long term objectives of the theory and modeling efforts are to provide improved fundamental basis for building appropriate constitutive equations for the description of the mechanical response of polymers to complex thermo-chemical-mechanical histories.

\section{Accomplishments:}

Significant progress was made in the areas related to the structure of two-component systems and a debut has been made to develop models of the dynamics of glass forming or complex fluids. The specific areas of work were in: a) mixing of plate-like and rod-like molecules in solvent; b) adsorption of molecules of differing architectures onto surfaces; c) collapse of a polymer chain in a melt of other polymers; d) composition dependence of the glass transition temperature; e) off-lattice simulations of the dynamics of dense polymer systems; $f$ ) massively parallel simulations of relaxations in glass forming systems; g) structure development in chemically reacting blends.

\section{Mixing Plate-like and Rod-Like Molecules with Solvent}

The Flory-Huggins calculation was extended and improved for applications to systems which are mixtures of plates, rods and solvent. The purpose was to understand those phases which occur because of entropy frustration. Applications of the theory now include [1] rigid rods (liquid crystals), [2] plates (this work), [3] glasses (past and future work), [4] spin glasses. The hope is to understand the common thread of frustration in all these systems, and to formulate a universal view. There are applications of this work to industry. A mixture of $5 \%$ layered silicate and $95 \%$ nylon is being used as timing gasket covers in Japanese autos. Dimensional stability under repeated thermal cycling is improved in these materials. Layered silicates are also being studied for use as flooring materials.

Work was completed which generalized the lattice model of polymers to treat systems of compact molecules such as plates, cubes and thick rods. The calculation allows for various concentrations, molecular weights, and molecular orientations. The thermodynamic properties of mixtures of plates and solvent have been calculated. This system shows a phase transition from randomly disordered to aligned plates that is much like the isotropic to nematic phase transition in rigid rod polymers. For squares of edge length to thickness ratio $r$ the transition to aligned phase occurs at a volume fraction $\mathrm{V}_{\mathrm{r}}$ given by $\mathrm{rV}_{\mathrm{r}}=2.7$, while for rigid rods of length $\mathrm{x}$ the relation is $\mathrm{xV}_{\mathrm{x}}=5$.

\section{Chemical Reactions Coupled to Spinodal Decomposition in Polymers}

This effort seeks to understand the coupling of chemistry to phase transition phenomena. The impact occurs whenever chemical change modifies the kinetics and equilibrium properties of polymers during processing. Our treatment concerns only the control of morphology by chemistry during spinodal decomposition. 
The basic equations for spinodal decomposition of polymers undergoing chemical reaction were derived. These basic equations can then be solved for various kinds of chemical reactions. The problem is computer intensive because of the nonlinear character of the equations.

\section{Collapse of a Polymer in a Matrix of Other Polymers}

The task is to determine the shape of one molecule in the matrix of other polymers. Lattice model statistics done carefully give the unexpected result that the Flory approximation is sufficient to yield a tricritical point.

\section{Glass Temperature Depression by use of Two Component Plasticizers}

The object is to determine the glass temperature depression by mixed plasticizer systems as a function of pressure, molecular weight of polymer, and plasticizer composition. The initial rate of glass temperature depression is a universal function of total mole fraction and does not depend on the kind of plasticizer provided only that the plasticizing molecules are small and that they dissolve in the polymer. The main conclusion that only the total mole fraction is relevant has not yet been tested experimentally. The entropy theory of glasses is used which in past work has been successful in locating the glass transition temperature.

\section{Computer Simulation Studies of the Dynamical Behavior of Dense Polymer Systems}

The processibility of plastics and the final characteristics of their fabricated end-products depend in large part upon the relaxation behavior of the high-polymer chains of which these materials are primarily composed. Better theoretical treatments of polymeric systems will lead to more efficient processing and to better fabricated end products. A major shortcoming of present theories is their inability to treat excluded volume and chain entanglement effects in a realistic way. Present work is aimed primarily at the study of these effects in dense (many-chain) systems by simulation of simple models of such systems on large computers. The simulation results provide data on simple model systems which serve to guide the development of better theoretical treatments, leading eventually to improvement of physical properties of plastic materials and products.

Two kinds of systems are under study at present: One-component glasses and twocomponent blends. The work on one-component glasses is in its early stages. Its initial emphasis is on the study of sample volume (i.e., density) as a function of temperature above and below the glass transition, and on the estimation of glass transition temperature as a function of quench rate. Simulation programs for these systems have been written and are being tried out.

The work on two-component blends is primarily directed at the study of phase separation following quenching. Following experimental practice, the simulations employ two techniques to observe phase separation: Angular dependence (form factor) of the scattering 
of light and/or neutrons, and "simulated microscopy"-- i.e., direct viewing of the patterns formed during phase separation. In the scattering studies, phase separation following quenching is indicated by the appearance of a peak in the scattering form factor. Its position and height appear to be governed by quite different time scales. In one example, the position of the peak reaches essentially its final value in approximately one-fifth the time required for it to reach its final intensity. The process appears to proceed in two stages - an initial, relatively rapid separation, followed by a much slower "ripening" period of coalescence and growth. Direct visual examination of the conformations of the system shows similar phenomena, using programs which allow display of both still pictures and movies of the phase-separating system.

\section{Relaxation in Complex and Glass-Forming Materials}

The physical properties of glasses are observed to evolve over very long time scales, leading to many important technological problems associated with product durability and mechanical failure. Presently, simulation techniques and increases in computational speed have developed to an extent that should allow an investigation of the evolution of microstructure in these complex materials, which is the origin of physical aging and the unusual and often universal viscoelastic properties of these materials. New theories suggest that many of the universal properties of glasses and other complex materials can be understood in terms of simple statistical mechanical models. The goal of our theoretical and computational investigations is to identify and verify a minimal model of the glass transition, and apply this model to materials processing problems.

A workshop on this topic, sponsored by the NIST Center for Theoretical and Computational Materials Science, is currently being organized for February, 1995.

In an effort to bring high performance computing to bear on elucidating the nature of glass-forming materials and the kinetic processes governing their structural recovery and viscoelastic properties, a joint effort is currently developing massively parallel molecular dynamics algorithms in collaboration with researchers at Sandia National Lab, Arizona State University and the University of Mainz. This approach will enable the study of glassy behavior of both monatomic and polymeric materials, and materials near surfaces, or within pores. Concurrently, we are exploring the applicability of newly-developed frustrated percolation models to the glass transition in collaboration with leading university researchers.

\section{Reaction-Controlled Morphology of Phase-Separating Materials}

The goal of this work is to understand the role of chemical reactions in stabilizing and tuning the morphology of phase-separating polymer blends, and to apply this knowledge to polymer processing and materials design.

The study of a Langevin model that was proposed for a phase-separating polymer blend undergoing chemical reaction has continued. Because of the high nonlinearity of the coupled 
equations that describe such a system, massively parallel computer simulation techniques were developed that allow the study of very large systems of molecules out to very long time scales. Both linear and self-consistent theoretical techniques, massively parallel numerical simulations of the nonlinear equations, and Monte Carlo simulations were used to show that chemical reactions can suppress the initial long-wavelength instability characteristic of spinodal decomposition, and stabilize the phase-separation process. The results suggest that simple chemical reactions in a phase-separating mixture give rise to an effect analogous to that seen in phase-separating systems where long-range and short-range forces compete, and that chemical reactions can be used to stabilize and tune transient, nonequilibrium patterns created in phase-separating materials. This work has enormous applicability to the processing of polymer materials, and promises to provide insight into the effectiveness of chemical reactions to control and stabilize blend morphology during processing. Generalization of the model to more complicated chemical reactions where, e.g., phase separation is induced by polymerization or arrested by transesterification is underway.

\section{Terminally Anchored Chain Interphases}

\section{J.H. van Zanten}

\section{Objective:}

The objective is to develop a simple, computationally transparent approach for describing the physics of terminally anchored chain interphases in contact with a multicomponent, size polydisperse solvent.

\section{Accomplishments:}

A Flory-Alexander mean field type description of terminally anchored chain interphases in contact with a multicomponent, size polydisperse solvent was developed wherein the effects of chain stretching were described in terms of freely jointed chain model (i.e., the inverse Langevin function) and the mixing effects were accounted for by the well-known FloryHuggins theory of polymer solutions. The chain stretching approach used here accounts for the finite extensibility of the terminally anchored chains, and, therefore, can be used to describe strongly stretched, high density interphases. Mass conservation and geometric considerations were used to account for the effect of solvent size. The equilibrium properties of the terminally anchored chain interphase were found to depend on the surface density of the anchored chains, the solvent composition (i.e., size and quality effects) and the chain size. ${ }^{1,2}$ As an example of the utility of this approach, the chromatographic properties of terminally anchored chain interphases were investigated within the developed model. ${ }^{2}$ Other potential applications are colloidal stabilization, surface modification, drug delivery, separations and catalysis.

Future work in this area will entail further refinements of the present theory, including the use of a more accurate description of chain stretching at high extensions (worm-like chain model) and non Flory-Huggins approaches to account for the mixing phenomena. Since an 
important technical application of these interphases is in the stabilization of colloidal dispersions, the applicable colloidal forces will be described within the framework of the model described herein. In addition, the potential use of these interphases in chemical processes will be considered in the context of actual contacting configurations.

'J.H. van Zanten, Macromolecules, 27, 5052 (1994)

${ }^{2} \mathrm{~J} . H$. van Zanten, Macromolecules, 27, 6797 (1994) 


\section{OTHER AGENCY PROGRAMS}

\section{$\underline{\text { Goals }}$}

In response to requests from other federal agencies the Division conducts research on a reimbursable basis to solve specific needs of the requesting agency. The nature of these activities changes from year to year depending on national priorities. Specific projects include an ONR effort to develop neutron and x-ray reflectometry for characterization of thin polymer films and interfaces, part of the Halon Fire Suppressant Replacement Program at NIST supported by the US Armed Services and the Federal Aviation Administration. A three year effort sponsored by the National Archives and Records Administration to improve the storage environment for archival records, assistance to the Bureau of Consular Affairs, Department of State to evaluate candidate security laminates and a long term activity with the National Institute of Dental Research to develop improved dental restorative materials. The last mentioned project is included in the report on the Dental and Medical Materials Program.

\section{Protection of Archival Records}

\section{C.M. Guttman and W.R. Blair}

\section{Objective:}

The objective is to improve the micro-environment for storage of archival records by determining the absorption and diffusion of pollutant gases through boxes used in the storage of archival records.

\section{Accomplishments:}

Protection of records stored in the National Archives and Records Administration (NARA) from atmospheric pollutants has been a matter of long-standing concern. This research program currently involves the study of shielding mechanisms of containers commonly used in protecting archival materials from such materials as sulfur dioxide $\left(\mathrm{SO}_{2}\right)$ and nitrogen oxides.

The diffusion constants of $\mathrm{SO}_{2}$ and $\mathrm{NO}$ for six types of box board used to make archival boxes were measured. For the two samples provided by NARA diffusion constants were found to be about $1 \times 10^{-3} \mathrm{~cm}^{2} / \mathrm{sec}$. Four samples obtained from a commercial manufacturer had diffusion constants of $3 \times 10^{-3}$ to less than $1 \times 10^{-5} \mathrm{~cm}^{2} / \mathrm{sec}$. These data suggest that in many cases, diffusion of $\mathrm{SO}_{2}$ and $\mathrm{NO}$ through box board is mediated by the particular glue used to laminate the box board.

The absorption of $\mathrm{SO}_{2}$ and $\mathrm{NO}_{2}$ by box board was found to be very dependent upon the nature of the box board sample. Both permanent and nonpermanent binding of $\mathrm{SO}_{2}$ were 
observed. $\mathrm{SO}_{2}$ absorption in the range of 800 to 1600 micrograms of $\mathrm{SO}_{2}$ per gram of box board was found for the NARA box boards.

The absorption of NO by the same NARA box board was found to be considerably less than that by $\mathrm{SO}_{2}$. For the two NARA box boards studied less than 1 microgram of NO per gram of box board was absorbed.

\title{
Development of X-ray and Neutron Reflectivity Techniques Using Model Systems
}

\author{
Wen-li Wu, J.H. van Zanten, W.E. Wallace, P. Chin and G.T. Davis
}

\section{Objectives:}

Determine interaction of polymer and substrate by measuring physical properties of ultra-thin polymer films under co-sponsorship of the Office of Naval Research.

\section{Accomplishments:}

Polymers are used pervasively in the packaging of microelectronic components for applications such as adhesives for die attach, interlayer dielectrics, molding compounds, encapsulants, and tape carriers in tape automated bonding. Since many of the failures experienced in polymeric electronic packages are initiated at polymer interfaces with materials such as silicon, silicon dioxide, copper, aluminum, and other polymers, it becomes important to understand the nature of these interfaces and the parameters that control adhesion. In this project, the interfacial region is being studied by using X-ray and neutron reflectivity to measure the influence of interaction with the substrate on the physical properties of ultra-thin polymer films (50 to $500 \AA)$. Density profiles of atomic composition in the interfacial region can be determined as well as sensitive measurements of film thickness as a function of temperature from which glass transition temperature can be determined.

The thermal behavior of several polymers (polystyrene (PS), polymethylmethacrylate (PMMA) and poly-(2)-vinylpyridine $(\mathrm{P}(2)$-vinylpyridine $(\mathrm{P}(2) \mathrm{VP})$ ) in contact with sulfuric acid/NoChromix treated silicon substrates has been investigated via $\mathrm{X}$-ray and neutron reflectometry. Ultrathin $(<1000 \AA)$ films of the various polymers are produced by spin coating on the silicon substrates from fairly dilute solutions $(\leq 2 \mathrm{wt} \%)$. It is our belief that the silicon surface is essentially the native oxide with a small $(<1 \%)$ amount of hydroxyl groups present. The three polymers considered in these studies display the following affinity for this silicon surface: P(2)VP > PMMA > PS. The film thicknesses are determined as a function of temperature under high vacuum conditions. The glass transition temperatures of ultrathin PS films are greatly reduced from its bulk value, while the glass transition temperatures of PMMA and P(2)VP increase with decreasing film thickness. The observed thermal expansion behavior is similar. Above $T_{g}$, the PS thermal expansion is found to be what is expected from a constrained film based upon literature values of bulk thermal expansion, while PMMA and P(2)VP display thermal expansion coefficients which decrease 
with decreasing film thickness. In the extreme case of thin $\mathrm{P}(2) \mathrm{VP}$ films, no thermal expansion is observed below the now elevated $T_{g}$. All of these observations are in agreement with the earlier description of the various polymer-substrate interactions and highlight the importance of these interactions on the thermal behavior of polymer-substrate interfaces and, in turn, ultrathin polymer films.

Hydrogen passivated silicon surface is known to have a stronger adhesion to PS than the acid-cleaned surface. Studies of polystyrene on hydrogen-passivated silicon (111) surfaces have shown a suppression of the coefficient of thermal expansion (CTE) which varies with film thickness. For films below $100 \AA$ no thermal expansion was seen up to $150^{\circ} \mathrm{C}$. For films several hundred Ångstroms thick thermal expansion was observed but was substantially less than the bulk linear CTE. This thermal behavior of PS on hydrogen passivated silicon is rather similar to that of $\mathrm{P}(2) \mathrm{VP}$ on acid cleaned silicon surface. This similarity suggests that the elevation in glass transition temperature and the suppression in CTE are direct consequences of strong adhesion between polymers and the substrates. A direct measurement of the adhesion energy between those polymers and silicon with different surface treatment is in progress.

In addition to silicon, other surfaces with relevance to electronic packaging have also been included in this work; they include chromium, aluminum, and graphite. Characterization of these surfaces in terms of their depth composition has been completed, and the work to elucidate the thermal behavior of the overlaid polymer films is now in progress.

Progress has also been made in refining the theory of off-specular reflectivity. Verification of this refined theory is now being conducted using two different well-defined surfaces; silicon surface with evenly spaced aluminum lines and silicon surface with monodisperse gold spheres.

\section{Elastomer Seal Compatibility for Halon Fire Suppressant Replacements}

G.B. McKenna, W.K. Waldron Jr. and F. Horkay ${ }^{1}$

${ }^{1}$ Eötvös Lorand University, Budapest, Hungary

Objectives: This project is a part of the Halon Fire Suppressant Replacement Program at NIST supported by the US Air Force, Navy, Army and the Federal Aviation Administration. The current research was motivated by the fact that the bromine atom, which is credited with giving halon 1301 (bromotrifluoromethane or $\mathrm{CF}_{3} \mathrm{Br}$ ) its strong inhibition character, readily reacts in a catalytic cycle to destroy ozone molecules. By international agreement (Montreal Protocol), commercial production of $\mathrm{CF}_{3} \mathrm{Br}$ has ceased in order to protect the stratospheric ozone layer. No alternatives are available which have all the positive characteristics of $\mathrm{CF}_{3} \mathrm{Br}$. Hence, there is an urgency to finding a replacement chemical in order to maintain aircraft safety. The sponsors are concerned with two specific applications: protection of commercial and military aircraft engine nacelles and protection of military aircraft dry bay 
areas.

\section{Accomplishments:}

The FY93 objectives of the Halon Fire Suppressant Replacement Program were: 1. Determine the best two candidates for each application from the twelve gaseous agents proposed by the Air Force. 2. Identify the best practical alternative from chemicals not among the twelve. 3. Ascertain whether or not sodium bicarbonate powder is a viable option. The Elastomer Seal Compatibility portion of the program is concerned with identifying elastomer seal systems which are compatible with the alternative fluid agents.

In October 1993, three of the twelve proposed agents were selected for additional testing by the sponsor's Technology Transition Team. HFC-125 $\left(\mathrm{C}_{2} \mathrm{HF}_{5}\right)$ and HFC-227 $\left(\mathrm{C}_{2} \mathrm{HF}_{7}\right)$ would be considered for engine nacelle applications. FC-218 $\left(\mathrm{C}_{3} \mathrm{~F}_{8}\right)$ and $\mathrm{HFC}-125\left(\mathrm{C}_{2} \mathrm{HF}_{5}\right)$ would be considered for dry bay applications. In addition, it was recommended that iodotrifluoromethane $\left(\mathrm{CF}_{3} \mathrm{I}\right)$ be included in the testing programs for both applications because it was more effective than Halon 1301 in suppressing non-premixed flames. Sodium bicarbonate powder was the most efficient agent, but it was later decided that the operational difficulties were too severe.

Although elastomer seal compatibility did not drive these down-selection decisions, the results from FY93's research did provide differentiation among the agents in terms of their interactions with the elastomers and greases, and they were factored into the down-selection process. A major conclusion from the results of the durability measurements is that the 150 ${ }^{\circ} \mathrm{C}$ condition was too severe, i.e., property changes were extreme for most of the elastomers and greases. Subsequent information from the Air Force indicated that all-metal storage containers are used when temperatures are expected to exceed $70{ }^{\circ} \mathrm{C}$. Therefore, additional testing at lower temperatures was required. The objectives for FY94 have been to downselect the candidate fire suppressant agents from three to one for each application. These down-selection decisions are still pending. However, observations during our research gave early indications that there were potential corrosion, polymer degradation, and toxicity problems associated with $\mathrm{CF}_{3} \mathrm{I}$. In particular, swelling measurements with $\mathrm{CF}_{3} \mathrm{I}$ were discontinued because a solid residue developed on pressure vessel's view ports at $70{ }^{\circ} \mathrm{C}$. It was later concluded that the $\mathrm{CF}_{3} \mathrm{I}$ was impure and degraded creating $\mathrm{HI}, \mathrm{HF}$, and $\mathrm{I}_{2}$. The durability measurements were also discontinued because of degradation problem, i.e., the silicone and fluorosilicone elastomers were completely destroyed, all three greases were destroyed, and the stainless steel fixtures were corroded after 1 week exposures at $100{ }^{\circ} \mathrm{C}$. These observations were important because they resulted in the discontinuation of testing of $\mathrm{CF}_{3} \mathrm{I}$ by the entire research team for approximately three months until a purer supply of the chemical was obtained. Testing with a new lot of $\mathrm{CF}_{3} \mathrm{I}$ has resumed, but our observations indicate that the saturation vapor pressure of the chemical increases after exposures at elevated temperatures. We believe that this is an indication that the same degradation observed previously is occurring but to a lesser degree. More recently, cardiotoxicity and genotoxicity testing have revealed adverse effects associated with $\mathrm{CF}_{3} \mathrm{I}$, and consequently, it has been eliminated from consideration for any use in occupied spaces. 
Excessive swelling or deterioration of the elastomer seal (o-ring and its lubricant) in the fire suppressant storage container could lead to leakage of the agent leaving the system unready to respond in case of fire. Short term exposure experiments were conducted and data were generated on the proclivity of eleven of the twelve fluid agents (the twelfth chemical HFC-32 is flammable and was not considered) to alter the properties of various polymers (elastomers and lubricants). The compatibilities of the fire suppressant agents with commonly used elastomers and lubricants (greases) were characterized using two types of measurements.

The first type was based on solution thermodynamics and characterizes swelling due to sorption of the agent into the elastomer or grease. The degree of swelling was determined by measuring the solvent (agent) weight fraction $w_{1}$ in the polymer/agent system at various temperatures and vapor pressures. Using these weight fractions and the Flory-Huggins theory, a single polymer-solvent interaction parameter $\chi$ was calculated for each polymer/agent system at various temperatures. The $\chi$ values were used to characterize the compatibilities of the agents with the elastomers and greases. Small $\chi$ values correspond to good solubility or, for present purposes, bad compatibility.

The second type, durability measurements, produced data on residual mechanical (rheological) properties of the elastomers (greases) after exposure to the agents at elevated temperature and pressure $\left(150^{\circ} \mathrm{C}, 5.86 \mathrm{MPa}\right)$. Compatibility ratings were based on the results of compression set and tensile test measurements of the elastomers and viscosity measurements of the greases. These tests provide direct information on the physical and chemical damage to the samples at extreme conditions.

In order to better understand the changes in mechanical properties with respect to temperature, three temperatures were considered: $50,75,100{ }^{\circ} \mathrm{C}$. Compression set measurements and tensile testing were performed for exposures to the four agents at each temperature for 1,2, and 4 weeks. The compression set and decrease in ultimate elongation were plotted $v s$. exposure time on log-log scales. The data were fit using a KWW stretched exponential creep function with the intention of extrapolating to longer exposure times. The analysis of the compression set measurements showed some differentiation but the results for the tensile test data were inconclusive. Additional rheological measurements were not performed for the greases because of the absence of systematic time dependencies for the viscosities at $150^{\circ} \mathrm{C}$. This suggests that no significant chemical degradation occurs for the three greases considered.

An activation energy analysis was used to determine the temperature dependencies of these durability measurements and the temperature of $75^{\circ} \mathrm{C}$ was chosen for additional long-term exposures. Long-term exposures for $8,16,32,48$, and 74 weeks at $75^{\circ} \mathrm{C}$ have been started and the results of compression set measurements and tensile testing are being used to check and improve the creep models. These long-term durability measurements and analyses will provide reliable estimates of the elastomers' resistance in service conditions.

Additional swelling measurements have been proposed to investigate the perfect mixing 
assumption for polymer solutions and gels, i.e., the volume of the swollen polymer equals the sum of the individual volumes of polymer and agent. This assumption is necessary to calculate the volume fractions of polymer and agent from their respective weight fractions and is an important assumption when measuring volume fractions directly. In addition, swelling measurements of polymers in supercritical solvents are being prepared.

\title{
Development and Utilization of Test Methods for Qualification of Passport Laminates
}

\section{W.G. McDonough and D.L. Hunston}

The Bureau of Consular Affairs at the U.S. Department of State is responsible for the U.S. passports, and this responsibility includes choosing the plastic laminate that is used to protect the photograph and descriptive information on the inside of the front cover. This section of the passport is called the data page. The laminates must protect the data page from dirt, spills and abrasions that may occur in normal use. Furthermore, these laminates must resist attempts to tamper with the photograph or data page. As part of the solicitation process for new passports, laminating materials are selected. The objective in this program is to provide assistance to the State Department in the laminate selection process. The responsibilities include helping to prepare the evaluation specifications for the solicitation, performing appropriate evaluation tests as part of the solicitation, examining new materials and security concepts provided by suppliers as candidates for future passports, providing feedback on new materials to help the suppliers improve their materials and to help the State Department decide on future directions, and developing new or modified test methods that will be appropriate for the next generation laminates.

During the past year, technical evaluations have been completed for two solicitations, and the plans for a third evaluations in the coming year have been prepared. Currently, NIST performs nine of the ten laminate tests used in the evaluation. Based on the expertise and results gained from these evaluations, major changes are now underway in the composition of future passports, and revised tests to accommodate these changes are being developed. The continuation of this work assures a smooth introduction of new technologies and materials in this vital application.

\section{Monitoring Polymer Processes through Fluorescence Probes}

\author{
F.W. Wang, R.E. Lowry and B.M. Fanconi
}

\section{Objectives:}

In the manufacture of a variety of products, such as polymer matrix composites and vinyl polymers, the cure or polymerization reactions of a polymer-forming system must be adequately monitored and controlled to produce the desirable resultant product. The objectives are to develop and refine fluorescence techniques to monitor chemical and physical 
changes in reactive polymer systems.

\section{Accomplishments:}

Cure of Epoxy Resins

Fluorescence techniques are particularly useful for cure monitoring because they are sensitive and adaptable to in situ, non-destructive monitoring. For example, Wang, Lowry and Fanconi used a fluorescence technique to monitor the cure at $60 \mathrm{C}$ of an epoxy resin which was a stoichiometric mixture of diglycidyl ether of bisphenol A(DGEBA) and 4,4'methylene-bis-(cyclohexylamine)(PACM). They dissolved in the epoxy resin 1-(4dimethylaminophenyl)-6-phenyl-1,3,5-hexatriene(DMA-DPH), which is a viscosity sensitive fluorescent probe, and 9,10-diphenylanthracene(DPA), which is an internal standard not sensitive to viscosity. They then measured the fluorescence intensities of the probe, DMADPH, and the internal standard, DPA, at various cure times. Finally, they used the ratio of these intensities, which is insensitive to the geometry of the sample or the presence of fillers, to monitor the cure of the epoxy resin.

Lin and Wang described a wavelength-shift method for fluorescence cure monitoring, in which they measured the change in Stokes' shift (the difference, $v_{\mathrm{A}}-v_{\mathrm{F}}$, where $v_{\mathrm{A}}$ and $v_{\mathrm{F}}$ are the peak wavenumbers of the absorption and fluorescence spectra of the fluorescent probe) to monitor polarity change and gelation during epoxy cure. The wavelength-shift method is especially advantageous for in situ, non-destructive cure monitoring, because it eliminates an internal standard probe which is often required in methods based on measurement of fluorescence intensity change, and it obviates a complex optical system which is used in methods based on measurements of fluorescence anisotropy change. However, in some situations, the wavelength-shift fluorescent probe used by Lin and Wang 1-(4-dimethylaminophenyl)-6-phenyl-1,3,5-hexatriene (DMA-DPH), has some limitations because its change in Stokes' shift is moderate and its excitation wavelength is not in the visible, where the interference of impurity fluorescence from the resin and the optic fiber is less serious than in the UV.

Recently, however, a class of wavelength-shift probes which have much better fluorescence properties for cure monitoring than DMA-DPH has been identified. Each of these wavelength-shift probes absorbs visible light and, in response to the change in polarity of its surroundings, exhibits a much larger change in Stokes' shift than DMA-DPH. Five of these wavelength-shift probes have been successfully used to monitor the cure of epoxy resins, by measuring the peak absorption and fluorescence frequencies of each probe at various cure times.

Fluorescence Monitoring of Poly(dimethylsiloxane) Network Formation

Manufacturing of pre-ceramic components, as well as preparation of adhesive joints, often involves the hydrosilation reaction of a vinyl-terminated polydimethylsiloxane polymer(vinyl silicone) with a dimethylsiloxane-methylhydrosiloxane copolymer(hydrosilicone). A 
fluorescence method was successfully developed to meet the need for online cure monitoring of these processes.

A trace amount of vinyl anthracene(VA) or 9,10-bis-(phenylethynyl)anthracene(BPEA) was added as a fluorescent probe to a resin consisting of the vinyl silicone, the hydrosilicone, and platinum divinyl complex, a catalyst. These probes were chosen because hydrosilation of the vinyl group in VA or the ethynyl groups in BPEA led to marked changes in the fluorescence properties of VA or BPEA. Furthermore, if the hydrosilation rate of the vinyl group in VA or the ethynyl groups in BPEA is comparable to the hydrosilation rate of the vinyl groups in the vinyl silicone, the changes in fluorescence properties will be suitable indicators of the hydrosilation or the network formation from the vinyl silicone and the hydrosilicone.

With the use of VA as a fluorescent probe, fluorescence spetra were obtained (at an excitation wavelength of $350 \mathrm{~nm}$ ) at three stages of cure: at the beginning of the cure at 60 $\mathrm{C}$ (Stage I), after 300 minutes of thermal treatment at $60 \mathrm{C}$ (Stage II), and after an additional thermal treatment at $130 \mathrm{C}$ for $17 \mathrm{~h}$ (Stage III). In the fluorescence spectrum taken at Stage II, the bands at $390 \mathrm{~nm}, 413 \mathrm{~nm}$ and $436 \mathrm{~nm}$ which are characteristic of methylanthracene appeared. The spectrum taken at Stage III was practically identical to that of methylanthracene, indicating the complete hydrosilation of the vinyl group. Since the fluorescence intensity at $394 \mathrm{~nm}$ remained constant all through the cure, the ratio of the intensity of the peak near $410 \mathrm{~nm}$ to the intensity at $394 \mathrm{~nm}$ was used as an indicator of the extent of hydrosilation. The ratio decreased monotonically with the cure, being $3.1,2.0$ and 1.7 at Stages I, II and III, respectively.

With the use of BPEA as a fluorescent probe, fluorescence spectra were obtained (at an excitation wavelength of $350 \mathrm{~nm}$ ) at three stages of cure: at the beginning of the cure at 60 $\mathrm{C}$ (Stage I), after 240 minutes of thermal treatment at $60 \mathrm{C}$ (Stage II), and after additional thermal treatment at $130 \mathrm{C}$ for $17 \mathrm{~h}$ (Stage III). The spectrum taken at Stage I had a band at $468 \mathrm{~nm}$, another band at $497 \mathrm{~nm}$, and a shoulder at $443 \mathrm{~nm}$, with relative intensities of 7, 5, and 1, respectively. In the spectrum taken at Stage II, the shoulder at $443 \mathrm{~nm}$ in Stage I became a distinct band which had a higher intensity than the band at $468 \mathrm{~nm}$. In addition, a shoulder appeared at $435 \mathrm{~nm}$. The spectrum taken at Stage III showed a shoulder at $468 \mathrm{~nm}$, where the most prominent band had appeared in Stage I. In addition, there was a broad band in the region where the shoulder at $435 \mathrm{~nm}$ had appeared in Stage II. The ratio of the intensity at $468 \mathrm{~nm}$ to the intensity at $443 \mathrm{~nm}$ decreased monotonically with the cure, being $7.0,0.81$ and 0.68 at Stages I, II and III, respectively.

Synthesis of Pyrene-labeled Poly(methyl methacrylate)

A three-step synthetic procedure was used to prepare a pyrene-labeled poly(methyl methacrylate) polymer, in order to meet a need of the Consortium on Measurement Technology For Polymer Processing. The procedure entailed the reduction of pyrenecarboxaldehyde to form pyrenylmethanol, the esterification of pyrenylmethanol to form pyrenylmethyl methacrylate, and copolymerization of pyrenylmethyl methacrylate and methyl 
methacrylate to form a pyrene-labeled poly(methyl methacrylate) polymer(py-PMMA). The fluorescence spectrum of a dilute solution of py-PMMA showed both pyrene fluorescence and pyrene excimer fluorescence.

\section{Scattering from Surfactant Vesicles and Vesicular Dispersions}

\section{J.H. van Zanten}

\section{Objective:}

The objective is to develop scattering techniques for the characterization of vesicular and micellar dispersions due to their increased utilization in various industries and applications such as biotechnology, pharmaceuticals, cosmetics and chemical separations, synthesis and catalysis. Non-invasive, rapid and economical optical characterization techniques are of special interest here.

\section{Accomplishments:}

The use of simultaneous, multiangle static light scattering spectroscopy in the characterization of vesicles has been considered in detail. Applications of the Rayleigh-Gans-Debye (RGD) theory of light scattering to the analysis of static light scattering spectra has determined that phosphatidylcholine vesicles prepared by detergent dialysis are essentially optically isotropic hollow spheres. ${ }^{1}$ In addition, static light scattering techniques have been used to estimate the wall thickness of phosphatidylcholine vesicles. ${ }^{1,2}$ This determination was done in two different manners. One was via a combination of the RGD theory and Zimm's light scattering technique ${ }^{1}$ while the other was done solely within the context of Zimm's light scattering technique. ${ }^{2}$ Lastly, a comprehensive review of the application of light, neutron and $\mathrm{x}$-ray scattering techniques to the characterization of vesicles and vesicular dispersions was prepared. ${ }^{3}$

Future work will investigate the applicability of scattering techniques to characterize vesicular and micellar dispersions. An approach utilizing Zimm's light scattering technique to estimate size polydispersity in these systems is under development. Also, the influence of size polydispersity and shape fluctuations on dynamic light scattering spectra and the use of multiangle scattering techniques are under consideration. It is hoped that in the future, NIST neutron scattering facilities can be utilized to thoroughly characterize a vesicular dispersion.

'J.H. van Zanten and H.G. Monbouquette, J. Coll. Interface Sci., 165, 512 (1994).

${ }^{2}$ J.H. van Zanten, Langmuir, 10, 4391 (1994).

${ }^{3}$ J.H. van Zanten, to appear in "Vesicles", edited by M. Rosoff, Marcel-Dekker. 


\section{OUTPUTS/INTERACTIONS}

\section{$\underline{\text { Publications }}$}

Antonucci, J.; Stansbury, J. Molecularly designed dental polymers. Book Chapter in Functional Polymers for Emerging Technologies [Editor], Imperial College, London published by the American Chemical Society, in press.

Antonucci, J.M.; Bennett, P.S.; Code, J.E. Effect of redox metals on dentin bonding with PMDM/N-phenyliminodiacetic acid. Proc. of IADR Meeting, Seattle, WA, 3/9-13/94, Abst. \#1404, Journal of Dental Research 73 (Special Issue), 277 (1994).

Code, J.E.; Antonucci, J.M.; Bennett, P.S. Photoactivated dentin bonding with Nphenyliminodiacetic acid. Proc. of IADR Meeting, Seattle, WA, 3/9-13/94, Abst. \#975, Journal of Dental Research 73 (Special Issue), 223 (1994).

Reed, B.B.; Antonucci. J.M.; Stansbury, J.W. Synthesis of a vinyl cyclic ketal derivative of camphorquinone. Proc. of IADR Meeting, Seattle, WA, 3/9-13/94, Abst. \#204, Journal of Dental Research 73 (Special Issue), 127 (1994).

Antonucci, J.M.; Wang, F.W.; Liu, D.W.; Tesk, J.A. Fluorescence cure monitoring of dental resins. Proc. of IADR Meeting, Seattle, WA, 3/9-13/94, Abst. \#1541, Journal of Dental Research 73 (Special Issue) (1994).

Schumacher, G.E.; Antonucci. J.M.; Bennett, P.S. N-phenyliminodiacetic acid as an etchant/primer for dentin bonding. Proc. of IADR Meeting, Seattle, WA, 3/9-13/94, Abst. \#1403, Journal of Dental Research (Special Issue), 277 (1994).

Antonucci, J.M.; Skrtic, D.; Eanes, E.D. Bioactive polymeric dental materials based on amorphous calcium. ACS Polymer Preprint $\underline{35}$ (2), 460-461 (1994).

Matsuya, Y.; Matsuya, S.; Antonucci, J.M.; Takagi, S.; Chow, L.C. Formation of hydroxyapatite in a polymeric calcium phosphate cement. Proceedings, First International Conference on Composite Engineering (ICCE/1), August, 861-862 (1994).

Skrtic, D.; Eanes, E.D.; Antonucci, J.M. Polymeric calcium phosphate composites with remineralization potential. Proc. of ACS Meeting, San Diego, CA, 3/13-17/94, in press.

Barnes, J.D. X-ray scattering in the intermediate angle range-challenges and opportunities. PMSE Preprints, 71, 91-2 (1994). 
Bauer, B.J.; Briber, R.M. The effect of crosslink density on phase separation in interpenetrating polymer networks. Advances in IPN's, Vol. 4, pp. 45-76 (1994).

Bauer, B.J.; Briber, R.M.; Dickens, B. "Grafted Interpenetrating Polymer Networks", ACS Books, Advances in Chemistry Series No. 239, pp. 179-204 (1994).

Blair, W.; Jewett, K.; Wang, F.; Schiller, S. Preparation and monitoring of lead acetate containing drinking water solutions for toxicity studies. NISTIR 5388.

Al-Sheikhly, M.; Farahani, M.; Bowen, R.L. Polymerization initiative by N-p-Tolylglycine: free radical reactions studied by pulse and steady-state radiolysis. Journal of Applied Polymer Science, 54: 1049-1058 (1994).

Thomas, Charles; Bur, A.J.; Adebo, Ade Ultrasonic measurement of polymer material properties during injection molding. Proc. SPE Ann. Technical Meeting, May 1994, p. 2236.

Bur, A.J.; Thomas, C.L. Real-time optical monitoring of polymer injection molding. Proc. SPE Ann. Technical Meeting, May 1994, p. 490.

Bur, A.J.; Wang, F.W.; Thomas, C.L.; Rose, J.L. In-line optical monitoring of polymer injection molding. Polym. Engr. \& Sci., 34, 671 (1994).

Carrier, D.D.; Kelly, J.R. In-ceram failure behavior and core-veneer interface quality as influenced by residual infiltration glass. J. Prosthodont, in review.

Carey, C.M.; Riggan, Jr., W.B. A cyclic polyamine ionophore for use in a dibasic phosphateselective electrode. Analytical Chemistry 66(21), 3587-3591 (1994).

Chang, S.S. Calorimetric studies on glasses and glass transition phenomena. ASTM Symposium on Assignment of the Glass Transition, in press.

Shearer, B.B.; Naleway, C.A.; Chow, L.C.; Chou, H. Dissolution characteristics of NaF- and $\mathrm{DCPD} / \mathrm{NaF}$-treated hydroxyapatites under bacterial acid challenge. Caries Research, in press.

Chow, L.C.; Takagi, S.; Shern, R.J.; Chow, T.H.; Takagi, K.K.; Sieck, B.A. Effects on whole saliva of chewing gums containing calcium phosphates. J. of Dental Research, $\underline{73}(1), 26-$ 32 (1994).

Chow, L.C.; Takagi, S.; Ishikawa, K. Formation of hydroxyapatite in cement systems. Proceedings of the Materials Research Society Symposium, Hydroxyapatite and Related Compounds (CRC Press, pp. 127-137 (1994). 
Chiang, M.Y.; McKenna, G.B.; Yuan, J. A micro-mechanical model of dimensional recovery in a composite layer. ANTEC 94, Soc. of Plastics Engineers, Vol. II, 2116 (1994); Polym. Engr. Sci., in press.

Chiang, M.Y.; McKenna, G.B.; Yuan, J. A viscoelastic micro-mechanical analysis for dimensional stability of a composite layer. Polym Eng Sci, in press.

Dadmun, M.; Han, C. The phase behavior of a hydrogen bonding molecular composite. Materials Research Society Symposia Series, in press.

Dadmun, M.; Muthukumar, M.; Han, C.; Hemplemann; Schwahn, D.; Springer, T. An investigation of the gelation of poly(benzyl L-glutamate) in Benzyl Alcohol Using Scattering Methods. Macromolecules, in press.

Bauer, S.; DeReggi, A.S. Thermal-pulse investigation of poled nonlinear optical polymers. 8th International Symposium on Electrets, Paris, France, Sept. 1994, pp. 218-223 (IEEE Pub. \#94CH3443-9).

DeReggi, A.S.; Giacometti, J.; Davis, G.T. Polarization distribution and stability in NLO polymers. Proceedings of SPIE Symposium on Organic, Metallo-Organic, and Polymeric Materials for Nonlinear Optical Applications, SPIE 2143, pp. 217-228 (1994).

Giacometti, J.; Minami, G.; DeReggi, A.S.; Dickens, B.; Chinaglia, D.L. Thermal pulse measurements on electron-beam irradiated fluoroethylene-propylene copolymer. Proceedings of 8th Inter. Symp. on Electrets, Paris, France, Sept. 1994, pp. 212-217 (IEEE Pub. \#94-CH34439).

DeReggi, A.S.; Giacometti, J.A. Poling kinetics in amorphous polar polymers containing space charge. 1994 Annual Report, Conference on Electrical Insulation and Dielectric Phenomena, pp. 286-291 (IEEE Pub. No. 94-CH3456-1).

Zanin, M.; Giacometti, J.A.; DeReggi, A.S. Charge injection and polarization in ethylenepropylene copolymer (EPDM) probed with the thermal pulse technique. 1993 Annual Report, Conference on Electrical Insulation and Dielectric Phenomena, pp. 215-220 (IEE Pub. \#93CH3269-8).

Giacometti, J.A.; DeReggi, A.S.; Davis, G.T.; Dickens, B. Thermal pulse study of the electric polarization in P(VDCN-VAc). 1993 Annual Report, Conference on Electrical Insulation and Dielectric Phenomena, pp. 275-280 (IEEE Pub. \#93-CH3269-8).

Balizer, E.; Federly, J.; Haught, D.; Dickens, B.; DeReggi, A.S. FTIR and X-ray study of polymorphs of nylon-11 and relation to ferroelectricity. J. Polym. Sci. Polym. Phys. Ed., $\underline{32}$, 365-369 (1994). 
DiMarzio, E.A. Contribution to a liquid-like theory of rubber elasticity: II Existence of a $\left(\lambda_{x} \lambda_{y}\right.$ $\left.+\lambda_{y} \lambda_{z}+\lambda_{z} \lambda_{x}\right)$ term. Polymer, in press.

DiMarzio, E.A. plus 10 other authors. Hierarchical Structures in Biology as a Guide for New Materials Technology. NMAB-464, National Academy Press, published 1994.

Dickens, B. Monitoring the cure of thin-layer polyimides on glass substrates with photoacoustic infrared spectroscopy. Proceedings of the 1994 International Symposium on Microelectronics, pp. 501-506 (Int'1 Soc. for Hybrid Microelectronics, Reston, VA),

ISBN 0-93-815-41-6.

Dickens-Venz, S.; Takagi, S.; Chow, L.C.; Bowen, R.L.; Johnston, A.D.; Dickens, B. Physical and chemical properties of resin-reinforced calcium phosphate cements. Dental Materials, 10, 1049-1058 (1994).

Dunkers, J.; Ishida, H. Vibrational assignments of N,N-BIS(3,5-dimethyl-2-hydroxybenzyl) methylamine in the fingerprint region. Spectrochimica Acta, in press.

Douglas, J.F.; Friedman, A. Coping with complex boundaries. Mathematics in Industrial Problems, Part 7, Springer-Verlag, pp. 166-185 (1994).

Douglas, J.F.; Garboczi, E.J. Intrinsic viscosity and the polarizability of particles having a wide range of shapes. SIAM Review, in press.

Douglas, J.; Ishinabe, T. Self-avoiding walk contacts and random walk self-intersections in variable dimensionality. Physical Review E, in press.

Kholodenko, A.; Bearden, D.; Douglas, J. Influence of surface interaction and chain stiffness on polymer-induced entropic forces and the dimensions of confined polymers. Phys. Rev. E., 49, 2206 (1994).

Douglas, J.; Frantz, P.; Johnson, H.; Schneider, H.; Granick S. Regimes of polymer adsorption-desorption kinetics. Colloids and Surfaces, $\underline{86}, 251$ (1994).

Zhou, H-X; Szabo, A.; Douglas, J.F.; Hubbard, J.B. A Brownian dynamics algorithm for calculating the hydrodynamic friction and the electrostatic capacitance of an arbitrarily-shaped object. J. Chem. Physics, 100, 3821 (1994).

Douglas, J.; Zhou, H-X; Hubbard, J.B. Hydrodynamic friction and the capacitance of arbitarily-shaped objects. J. Phys. E., 49, 5319 (1994).

Douglas, J.; Freed, K. Competition between hydrodynamic screen ('draining') and excluded volume interactions in an isolated polymer chain. Macromolecules, 27, 6088 (1994). 
Kholodenko, A.; Douglas, J. Generalized Stokes-Einstein equation for spherical particle suspensions. Physical Review E, in press.

Douglas, J.; Hubbard, J.; Zhou, H-X Friction coefficient of polymers chains. Proceedings of American Chemical Society Meeting, Poly. Matls. Sci. \& Eng. Symposium, August (1994), p. 639.

Eanes, E.D.; Hailer, A.W. Effect of ultrafilterable fragments from chondroitinase and protease treated aggrecan on calcium phosphate precipitation in liposomal suspensions. Calicified Tissue International 55, 176-179 (1994).

Lyzak, W.A.; Flaitz, C.M.; McGuckin, R.S.; Eichmiller, F.C.; Brown, R.S. Diagnosis and treatment of an oral base-metal contact lesion following negative dermatologic patch tests. Annals of Allergy, $\underline{73}, 161-165$ (1994).

Eichmiller, F.C. An overview of beta quartz glass-ceramic inserts. Journees de Biomateriaux Dentiares, in press.

George, L.A.; Eichmiller, F.C. Glass-ceramic inserts for use in composite dental restorations. Proceedings, 13th Southern Biomedical Engineering Conference, J. Vossoughi, ed., University of the District of Columbia, Washington, DC, April 16-17, 1994, pp. 764-767 (1994).

Glotzer, S.C.; DiMarzio, E.A.; Muthukumar, M. Spinodal decomposition of chemically reactive binary mixtures. Physical Review Letters, in press.

Glotzer, S.C.; Stauffer, D.; Jan, N. Monte Carolo simulations of spinodal decomposition of chemically reacting binary mixtures. Physical Review Letters, 72 , 4109 (1994).

Glotzer, S.C.; Coniglio, A. Self-consistent solution of phase separation with competing interactions. Physical Review E, 므, 4241 (1994).

Glotzer, S.C.; DiMarzio, E.A.; Muthukumar, M. Spinodal decompostion of chemically reactive materials. ACS Preprint, August 1994 Meeting, Washington, DC, 71,645 (1994).

Ossadnik, P.; Gyure, M.F.; Stanley, H.E.; Glotzer, S.C. Molecular dynamics simulation of spinodal decomposition in a two-dimensional binary fluid mixture. Physical Review Letters, $\underline{72}$, 2498 (1994).

Guard, H.E.; Coleman, W.M.; Ross, M.M. On the characterization of the reaction of organotin compounds with $\mathrm{D}$-glucuronic acid. Carbohydrate Research, in press.

Guttman, C.M.; Maurey, J.R. Determination of the weight average molecular weight of two poly(ethylene oxides), SRM 1923 and SRM 1924. NISTIR 5286. 
Guttman, C.; Johnsonbaugh, D.; Jewett, K. Protection of archival records from pollutants III. Diffusion and absorption studies of archival boxboards exposed to ppm concentration. NISTIR 5404 (1994).

Guttman, C.; Douglas, J.; DiMarzio, E. Chromatography of macromolecules near the absorption transition: study of copolymers, comb polymers, and stars. Proceedings of the American Chemical Society, Division of Polymeric Materials: Science and Engineering Fall Meeting, Washington, DC, Vol. 71, 641 (1994).

Han, C. Mobility and dissolution of fluctuations in polymer mixtures. ACS PMSE Preprint, 71, 630 (1994).

Holoubek, J.; Han, C.C. Optical transmission of phase separating polystyrene/poly(methyl methacrylate). ACS PMSE Preprint, 71, 368 (1994).

Dadmun, M.; Han, C. A neutron scattering study of the orientation of a liquid crystalline polymer by shear flow. Macromolecules, 27, 7522 (1994).

Balsara, N.P.; Jonnalagadda, S.V.; Lin, C.C.; Han, C.C.; Krishnamoorti, R. Thermodynamics interactions and correlations in mixtures of two homopolymers and a block copolymer by small angle neutron scattering. J. Chem. Phys. 99, 10011 (1993)

Hobbie, E.; Bauer, B.; Han, C. Fractal growth during early-stage spinodal decomposition in a hydrogen-bonded polymer blend. Physical Review Letters 12, 1830 (1994)

Han, C.C.; Yoon, Hichang Statics and kinetics of phase behavior in polymer blends. NATAS (North American Thermal Analysis Society) Polymer Blends and Copolymers Symposium, p. 23 (1993).

Han, C.C. Shear rate dependence of the critical dynamics of polymer mixtures. ACS Polymer Preprint, $\underline{35}, 58$ (1994).

Hobbie, E.K.; Nakatani, A.I.; Han, C. Shear-induced mixing in polymer blends. Modern Physics Letters B, 19, 1143 (1994).

Jinnai, H.; Hasegawa, H.; Hashimoto, T.; Han, C.C. Time-resolved small-angle neutron scattering study of spinodal decomposition in deuterated and protonated polybutadiene blends: 1. Effect of initial thermal fluctuations. J. Chem. Phys., 99, 4845 (1993)

Yoon, H.; Feng, Y.; Qiu, Y.; Han, C.C. Structural stabilization of phase separating $\mathrm{PC} /$ polymer blends through interfacial modification by transesterification reaction. J. of Polymer Science, in press. 
Ishikawa, K.; Takagi, S.; Chow, L.C.; Ishikawa, Y.; Eanes, E.D.; Asaoka, K. Behavior of a calcium phosphate cement in simulated blood plasma in vitro. Dental Materials $\underline{10}, 26-32$ (1994).

Ishikawa, K.; Eanes, E.D.; Tung, M.S. A constant composition study of the effect of supersaturation on apatitie crystal formation in aqueous solutions at physiologic $\mathrm{pH}$ and temperature. Journal of Dental Research 73, 1462-1469 (1994).

Ishikawa, K.; Eanes, E.D. The hydrolysis of anhydrous dicalcium phosphate into hydroxyapatite in the presence of calcium chloride. J. of Dental Research $\underline{72}$, 474-480 (1993).

Jackson, C.L.; Muthukumar, M.; Barnes, K.A.; Morrison, F.A.; May, J.W.; Nakatani, A.I.; Han, C.C. Shear-excited morphological states in a triblock copolymer. American Chemical Society, Polymer Preprints, $\underline{35}, 624$ (1994).

Jackson, C.L.; Bauer, B.J.; Barnes, J.D. The morphology of interpenetating organic-inorganic polymeric networks. ACS Polymer Materials Science \& Engineering Preprints, 71,85 (1994).

Jackson, C.L.; Barnes, K.A.; Morrison, F.A.; Mays, J.W.; Nakatani, A.I.; Han, C.C. A shear-induced martensitic-like transformation in a block copolymer melt. Macromolecules, (February 13 issue), 1995, in press.

Karim, A.; Felcher, G.P.; Russell, T.P. The interdiffusion of polymers at short times. Macromolecules, Vol. 27, No. 23, p. 6973 (1994).

Karim, A.; Satija, S.; Douglas, J.; Ankner, J.F.; Fetters, L.J. Neutron reflectivity study of the density profile of a model end-grafted polymer brush: influence of solvent quality. Physical Review Letters, $\underline{73}$, No. 25, p. 3407 (1994).

Karim, A.; Satija, S.K.; Han, C.C.; Slawecki, T.M.; Kumar, S.K.; Russell, T.P. Phase behavior of thin polymer blend films. ACS PMSE Symposium Preprints, 71, p. 280 (1994).

Kelly, J.R. Clinical failure of dental ceramic structures: insights from combined fractography, in vitro testing and finite element analysis. Ceramics in Biomedical Applications: Proceedings American Ceramic Society, in press.

Harvey, C.K.; Kelly, J.R. Contact damage as a failure mode during in-vitro testing. J. Prosthodontics, in press.

Kelly, J.R. Extrapolation from strength: caveat emptro. Trans. Acad. Dent. Mater. 7, 16-22, (1994).

Kelly, J.R. Extrapolation from strength: caveat emptro. Dent. Mater., in review. 
Markovic, M.; Fowler, B.O.; Brown, W.E. Octacalcium Phosphate Carboxylates, IV. Incorporation of excess succinate and ammonium ions in the octacalcium phosphate succinate structure. Proceedings of Symposium, Hydroxyapatite and Related Compounds (CRC Press), 139-144 (1994).

Markovic, M.; Fowler, B.O.; Brown, W.E. Octacalcium Phosphate Carboxylates, IV. Kinetics of formation and solubility of octacalcium phosphate succinate. J. of Crystal Growth 135, 533538 (1994).

Mathew, M.; Takagi, S.; Fowler, B.O.; Markovic, M. The crystal structure of calcium succinate monohydrate. Journal of Chemical Crystallography 24(7), 437-440 (1994).

Mathew, M.; Wong-Ng, W. Crystal structure of a new monoclinic form of potassium dihydrogen phosphate containing orthophosphacidium ion, $\left(\mathrm{H}_{4} \mathrm{PO}_{4}\right)$. Journal of Solid State Chemistry, in press.

Maurey, J.M.; Guttman, C.M. Studies on the melt flow rate of SRM 1473, a low density polyethylene. NISTIR-1473.

McKenna, G.B. Dilatometric evidence for the apparent decoupling of glassy structure from the mechanical stress field. J. Non-Crystalline Solids, 172-174, 756 (1994).

McKenna, G.B. On the physics required for prediction of long term performance of polymers and their composites. Seminar Lectures in the 2nd Intl. Disc. Meeting on Relaxations in Complex Systems (in press) and J. Res. NIST, 99, 169 (1994).

McKenna, G.B.; Leterrier, Y.; Schultheisz, C.R. The evolution of material properties during physical aging in "Use of Plastics and Plastic Composites: Materials and Mechanics Issues", ed. by U.K. Stokes, ASME, New York, 261 (1993).

Spinu, I.; McKenna, G.B. Physical aging of Nylon 66. ANTEC, SPE, Vol. II, 2063 (1994).

Aboulfaraj, M.; G'Sell, C.; Mangelinck, D.; McKenna, G. B. Physical aging of epoxy networks after quenching and/or plastic cycling. J. Non-Crystalline Solids, 172-174, 615 (1994).

McKenna, G.B.; Schultheisz, C.R.; Leterrier, Y. Volume recovery and physical aging: dilatometric evidence for different kinetics. Deformation, Yield \& Fracture of Polymers, $\underline{9}$, p. $31 / 1$, Inst. of Materials, London.

McKenna, G.B.; Waldron, W.K.; Horkay, F. Swelling of elastomers in sub- and super-critical fluorocarbons. Deformation, Yield and Fracture of Polymers, 9 , p 111/4, Institute of Materials, London. 
McKenna, G.B.; Horkay, F. Effect of crosslinks on the thermodynamics of poly(vinyl alcohol) hydrogels. Polymer, in press.

McKenna, G.B. Glassy state relaxation and aging. Polymer Preprints, $\underline{352}, 90$ (1994).

Terech, P.; Rodriguez, V.; Barnes, J.D.; McKenna, G.B. Organogels and aerogels of racemic and chiral 12-hydroxy octadecanoic acid. Langmuir, in press.

McKenna, G.B.; Waldron, W.K.; Horkay, F. Section 8. Elastomer seal compatibility, "Evaluation of alternate in-flight fire suppressants for full-scale testing in simulated aircraft nacelles and dry bays", ed. by W. L. Grosshandler, R.G. Gann and W.M. Pitts, NIST SP861, April, 1994.

Horkay, F.; Waldron, W.K.; McKenna, G.B. Elastomer compatability with fluorocarbons by vapor sorption measurements. Polym. Engr. and Sci., 71, 735 (1994).

Ketcham, S.A.; Niemiec, J.M.; McKenna, G.B. Extension and compression of elastomeric butt joint seals. ASCE Engineering Mechanics Div. Journal, submitted.

Spinu, I.; McKenna, G.B. Physical aging of nylon 66. Polym. Eng. and Sci., in press.

Misra, D.N. Interaction of chlorhexidine digluconate with and adsorption of chlorhexidine on hydroxyapatite. Journal of Biomedical Materials Research 28, 1375-1381 (1994).

Misra, D.N. Interaction of some coupling agents and organic compounds with hydroxyapatite: hydrogen bonding, adsorption and adhesion. Journal of Adhesion Science and Technology $\underline{8}(2)$, 87-99 (1994).

Mopsik, F.I. Stability of a numerical Laplace transform for dielectric measurements. IEEE Transactions on Dielectrics and Electrical Insulation, 1, 3 (1994).

Mopsik, F.I. Radiation induced dielectric loss in hydrocarbon polymers. Polym. Sci.: Part B: Polym. Phys., 31, 1989 (1993).

Nakatani, A.I.; Douglas, J.F.; Ban, W.-B.; Han, C.C. A neutron scattering study of shear induced turbidity in polystyrene dissolved in dioctyl phthalate. J. Chem. Phys., 100, 3224 (1994).

Sung, L.; Nakatani, A.; Fauvarque, P.; Douglas, J.; Han, C. SANS studies of the phase behavior of binary polymer blend/diblock copolymer mixtures. ACS PMSE Preprints, $\underline{71}$ (1994), p. 370.

Parnas, R.S.; Cohen, Y. A terminally anchored polymer chain in shear flow: Consistent Calculations. Rheologica, in press. 
Parnas, R.S.; Howard, J.G.; Luce, T.L.; Advani, S.G. A proposed standard reference material for permeability. Polymer Composites, in press.

Luce, T.L.; Advani, S.G.; Howard, J.G.; Parnas, R.S. Permeability characterization, part 2: flow behavior in multiple-layer preforms. Polymer Composites, in press.

Advani, S.G.; Bruschke, M.V.; Parnas, R.S. Resin transfer molding in flow phenomena in polymeric composites. Book: Flow Phenomena in Polymeric Composites, ed.: S.G. Advani, Elsevier, p. 461.

Phelan, F.R.; Leung, Y.; Parnas, R. Modeling of microscale flow in unidirectional fibrous porous media. American Society for Composites 8th Technical Conference on Composite Materials, Journal of Thermoplastic Composite Materials, 7, p. 208-218, July 1994.

Phelan, F.R.; Wise, G. Analysis of transverse flow in aligned fibrous porous media. Composites Manufacturing, in press.

Woerdeman, D.L.; Phelan, F.R.; Parnas, R.S. Interpretation of 3-D permeability measurements for RTM modeling. Polymer Composites, in press.

Ratzker, M.; Lashmore, D.S.; Johnson, C.E.; Shapiro, A. Electroforming of metallic glasses for dental applications. Dental Materials, in press.

Ratzker, M.; Lashmore, D.S.; Dariel, M.P. Ambient temperature synthesis of bulk intermetallics. Materials Research Society Proceedings, 350, 41-46 (1994).

Rose, K.J.; Carey, C.M. Swimmer's calculus. Journal of Pediatric Exercise (1994).

Schen, M.; Davis, G.; Mopsik, F.; Guthrie, W. An industry/government/university partnership: measuring sub-micrometer strain in polymer films. Proceedings of IPC Printed Circuits Exposition, April 24-27, 1994, Boston, MA

Schultheisz, C.R. A note on the Huygens-Fresnel-Kirchhoff diffraction of spherical waves by a circular aperature. J. Optical Society of America A, Vol. 11, No. 2, February, 1994.

Schultheisz, C.R.; Waas, A.M. Compressive failure of composites. Progress in Aerospace Sciences, An International Review Journal, in press.

Schutte, C.L.; McDonough, W.; Shioya, M.; McAuliffe, M.; Greenwood, M. The use of a single-fiber fragmentation test to study environmental durability of interfaces/interphases between DGEBA/MPDA epoxy and a glass fiber: the effect of moisture. Composites, Vol. 25, No. 7, p. 617-624, 1994. 
Schutte, C.L.; McAuliffe, M. Durability of glass fiber/polymer composites. ANTEC 94, Society of Plastics Engineers, 2394-2397.

Schutte, C.L. Environmental durability of glass fiber composites, Materials Science Reports, Vol. R13, No. 7, pp. 265-323, Nov. 15, 1994.

Schutte, C.L. Use of the single-fiber fragmentation test to study durability of model glass fiber/epoxy interfaces/interphases. ICCE/1 1st Intl. Conf. on Composite Eng., New Orleans, LA, pp. 471-472.

Schutte, C.L.; McAuliffe, M. Use of the single-fiber fragmentation test to study durability of model glass fiber/epoxy interfaces/interphases. American Society of Composites 9th Technical Conference Proceedings, pp. 1028-1033.

Skrtic, D.; Eanes, E.D. Effect of 1-hydroxyethylidene-1,1-bisphosphonate on membranemediated calcium phosphate formation in model liposomal suspensions. Bone and Mineral $\underline{26}$, 219-229 (1994).

Smith, L.E.; Fanconi, B.M. Polymers Division Technical Activities-1993. NISTIR 5315.

Stansbury, J.W.; Dickens, B.; Liu, D.W. Preparation and characterization of cyclopolymerizable resin formulations. Journal of Dental Research, in press.

Stansbury, J.W.; Liu, D.W.; Kim, S.I. Polymer-supported catalysts for synthesis of cyclopolymerizable monomers. Polymer Preprints $\underline{35}(1)$, 474-475 (1994).

Terech, P.; Maldivi, P.; Dammer, C. "Living polymers in organic solvents: stress relaxation in bicopper tetracarboxylate/tert-butyl cyclohexane, in press.

Smith, T.B.; Kelly, J.R.; Tesk, J.A. Fracture behavior of In-Ceram ${ }^{\circledR}$ and metal-ceramic restorations. J. Prosthodont. $\underline{3}(3), 138-144$ (1994).

Tesk, J.A.; Okuno, O. Investments for processing of dental materials at high temperatures ( $\mathrm{T}$ ca. $\left.1200-1300^{\circ} \mathrm{C}\right)$. Book Chapter in Dental Materials: Properties and Selection, 2nd Ed., in press.

Tesk, J.A.; Antonucci, J.M.; Stansbury, J.W.; Liu, D.W.; Kelly, R.J.; Asaoka, K.; Code, J.; Schumacher, G. Properties and interactions of oral structures and restorative materials. NISTIR 5426.

Asaoka, K.; Tesk, J.A.; Kelly, J.R. Evaluation of fracture toughness and residual stress in dental porcelain by indentation-microfracture method. Proc. of the 4th International Conference on Residual Stresses, 6/8-10/94, Baltimore, MD, Society for Experimental Mechanics, 854-859 (1994). 
Tomazic, B.B. Characterization of mineral phases in cardiovascular calcification. Proceedings of Symposium, Hydroxyapatite and Related Compounds (CRC Press), 93-115 (1994).

Tomazic, B.B.; Brown, W.E.; Schoen, F.J. Physiochemical properties of calcific deposits isolated from porcine bioprosthetic heart valves removed from patients following 2-13 function. Journal of Biomedical Materials Research 28, 35-47 (1994).

Tung, M.S.; O'Farrell, T.J. Effect of ethanol on the formation of calcium phosphates - A simple method for preparation of amorphous calcium phosphate. J. of Crystal Growth, in press.

Tung, M.S.; Lin, C.; Chow, T.H.; Sung, P. The effect of ethanol on the solubility of hydroxyapatite in the system $\mathrm{Ca}(\mathrm{OH})_{2}-\mathrm{H}_{3} \mathrm{PO}_{4}-\mathrm{H}_{2}$ at 25 and $33^{\circ} \mathrm{C}$. Proceedings of the Materials Research Symposium, Hydroxyapatite and Related Compounds (CRC Press), pp. 145-151 (1994).

Marugan, M.M.; Perez, E.; VanderHart, D.L. Solid-state ${ }^{13}$ NMR study of thermotropic polybibenzoates. I. Poly(heptamethylene, p,p'-bibenzoate) and poly[oxybis(trimethylene)p,p'bibenzoate], Macromolecules, in press.

Hackney, J.M.; Kraemer, G.P.; Atalla, R.H.; VanderHart, D.L.; Chapman, D.J. Influence of hydrodynamic environment on composition and macro-molecular organization of structural polysaccharides in egregia menziesii cell walls. Planta, 192, 461-472 (1994).

VanderHart, D.L. Proton spin diffusion studies of polymers blends having modest monomer size: I. Poly(styrene)/Poly(xylylene ether) - a miscible blend. Macromolecules, 27, 2837-2845 (1994).

Verdier, P.H.; Kranbuehl, D.E. Phase separation in polymer blends: off-lattice simulation studies. Polymer Preprints (ACS), 35, 73 (1994).

Kranbuehl, D.E.; Verdier, P.H. Effects of variable excluded volume on the relaxation behavior of off-lattice polymer chains. Polymer Preprints (ACS), $\underline{35}, 71$ (1994).

Verdier, P.H.; Kranbuehl, D.E. Computer simulation studies of scattering from phaseseparating polymer blends. Polym. Mats.: Science \& Engineering, 71, 643 (1994).

van Zanten, J. Unilamellar vesicle diameter and wall thickness determined by Zimm's light scattering technique. Langmuir, 10, 4391 (1994).

van Zanten, J. Terminally anchored chain interphases: the effect of multicomponent polydisperse solvents on their equilibrium properties. Macromolecules 27, 5052 (1994).

van Zanten, J. Terminally anchored chain interphases: their chromatographic properties. Macromolecules 27, 6797 (1994). 
van Zanten, J. Characterization of vesicles and vesicular dispersions via scattering techniques. Book Chapter in Vesicles, M. Rosoff, ed., Marcel-Dekher, in press.

Wineman, A.S.; Waldron, W.K. Yield-like response of a compressible nonlinear viscoelastic solid. J. of Rheology, in press.

Wineman, A.S.; Waldron, W.K. Normal stress effects induced during circular shear of a compressible nonlinar elastic cylinder, Intl. J. Non-Linear Mechanics, in press.

Waldron, W.K.; McKenna, G.B.; Santore, M.M. Probing aging and rejuvenation in the nonlinear viscoelastic range. Soc. of Plastic Engineers Technical Papers, Vol. II, 2071 (1994).

Waldron, W.K.; McKenna, G.B.; Santore, M.M. The nonlinear viscoelastic respone and apparent rejuvenation of an epoxy glass. J. of Rheology, in press.

Leblanc, L.; Ross, G.; Wallace, W. Measured stopping powers of hydrogen and helium in polystyrene near their maximum values. Nuclear Instruments and Methods in Physics Res. B (in press).

Lin, K.-F.; Wang, F.W. Fluorescence monitoring of polarity change and gelation during epoxy cure. Polymer, in press.

Slomkowski, S.; Kowalczyk, D.; Basinska, T.; Wang, F.W. Proteins at the interfaces of poly(styrene/acrolein) latexes and solvent. ACS Symposium: Macro-ion Characterization: From Dilute Solutions to Complex Fluids, in press.

Kowalczyk, D.; Slomkowski, S.; Wang, F.W. Changes in conformation of human serum albumin (HSA) and gamma globulins ( $\mathrm{G}$ ) upon adsorption of polystyrene and poly(styrene/acrolein) latexes: studies by fluorescence spectroscopy. Journal of Bioactive and Compatible Polymers, in press.

Waterstrat, R.M.; Okabe, T. Dental Amalgam. Chapter in Intermetallic Compounds, J. Westbrook, Editor, John Wiley \& Sons, pp. 575-590 (1994).

Wu, W.L.; Orts, W.J.; Majkrzak, C.J.; Hunston, D.L. Water adsorption at a polyimide/silicon wafer interface. Polym. Eng. \& Sci., in press.

Wu, W.L.; Orts, W.J.; van Zanten, J.H.; Fanconi, B.M. Density profile of spin cast polymethylmethacrylate thin films. J. Polym. Sci.:13: Polymer Phys., 32, 2475 (1994).

Wu, W.L. Off-specular reflection from flat interfaces. J. Chem. and Physics 101(5), 4198 (1994) 
Wu, W.L. Water adsorption at polymer/silicon water interfaces. Materials Research Society Symp. Proc., $\underline{338}, 565$ (1994).

Wu, W.L.; van Zanten, J.; Orts, W. Observation of thickness dependent thermal expansion in ultrathin deuterated polymethylmethacrylate films on silicon. Macromolecules, in press.

Wu, W.L.; van Zanten, J.; Orts, W. Glass transition temperature of ultrathin polymer films on silicon. Proceedings of the 1994 International Symposium on Microelectronics, pp. 507-511 (Int'l Soc. for Hybrid Microelectronics, Reston, VA). 


\section{Technical and Professional Committees: Leadership Activities}

Academy for Dental Materials

J.A. Tesk, Member, Long Range Planning Committee

Academy of Operational Dentistry

F.C. Eichmiller, Chairman of Research Committee

American Academy of Fixed Prosthodontics

J.R. Kelly, Research Committee

American Academy of Gold Foil Operators

F.C. Eichmiller, Chairman of Research Committee, Executive Board Member

American Academy of Implant Dentistry

J.A. Tesk, J. Oral Implantology, Editorial Board

American Association for Dental Research - Washington Section

F.C. Eichmiller, Past President

L.C. Chow, Past President

J.W. Stansbury, Current President

J.R. Kelly, Vice-President

L.C. Chow, Member, Program Review Committee, Mineralized Tissue Group

American Association of Engineering Societies: Engineers' Precollege Education Council

L.A. George, Member

American Ceramic Society

L.A. George, Chair, Pre-College Education Sub-Committee

L.A. George, Vice-Chair, Baltimore/Washington Section

American Chemical Society

J.M. Antonucci, Councilor; Member of Admissions Committee

M. Farahani, Member

American College of Prosthodontists

J.R. Kelly, Research Committee

American Crystallographic Association

J.D. Barnes, 1995 Chair, Small Angle Scattering Special Intergroup 
American Institute of Chemical Engineering

R.S. Parnas, Programming Committee

C.L. Schutte, Session Chair

American Institute of Physics

G.B. McKenna, Governing Board Member

American National Standards Committee, MD 156, Dental Materials, Instruments, and Equipment

J.M. Antonucci

Observer-Participant, Task Force on Posterior Composites

Observer, Task Forces on Dental Gloves and Adhesion

R.L. Bowen

Secretary, Subcommittee on Biological Evaluation of Dental Materials

Member, Subcommittee on Direct Filling Resins

F.C. Eichmiller, Chairman, Subcommittee \#39 on Pit and Fissure Sealants

J.R. Kelly, Chairman, Subcommittee \#38 on Porcelain-Metal Systems

J.A. Tesk

Member, MD 156 Committee, NIST Representative

Member, Subcommittee \#38 on Porcelain-Metal Systems

Chairman, \#14, Base Metal Casting Alloys

Observer, \#5, Dental Casting Alloys

Observer, \#40, Dental Implants

Member, Task Force on Posterior Composites

Vice Chairman, Terminology

Vice Chairman, ASC MD 56, WG3, Terminology and Special Projects

American National Standards Institute

J.A. Tesk, Member, Medical Device Standards Board

American Physical Society

G.B. McKenna, Past-Chairman, Division of High Polymer Physics

C.C. Han, Executive Committee, Division of High Polymer Physics

Program Chair, Division of High Polymer Physics, 1995

American Society for Mechanical Engineers

M.A. Schen, Organizer and Editor, Symposium on Thermal and Mechanical Behavior and Modeling, November 1994

American Society for Testing and Materials (ASTM)

F.W. Wang

Member, D-20 Plastics

Member, D-20.95 Recycled Plastics

Member, D-20.96 Environmentally Degradable Plastics 
Member, G-3 Durability of Non-Metallic Materials

Member, G-3.05 Reference Materials for Exposure Tests

Member, E-13 Molecular Spectroscopy

Member, E-13,09 Fiber Optics

S.S. Chang

Member, D37 Thermal Measurements

Member, D37.01 Test Methods

Member, D37.05 Thermophysical Properties

M. Farahani

Voting Member, E10.01

C.M. Guttman

Member, D20.30 Thermal Properties

Member, D20.70 Analytical Methods

Member, E-37 Subcommittee on Thermal Analysis

Chairman, Task Group on Purity Standards and Methods

D.L. Hunston

Member, D30 High Modulus Fibers \& Their Composites

G.B. McKenna

Member, D-11 Rubber and Rubber-like Materials

Member, E-9 Fatigue

Armstrong World Industries

C.C. Han, Member, Advisory Board

G.B. McKenna, Member, Advisory Board

E.A. DiMarzio, Member, Advisory Board

Technical Agent for ATP Cooperative Agreement

Representative, External Advisory Board for ATP Cooperative Agreement

Calcified Tissue International

E.D. Eanes, Member, Editorial Board

Case Western University/Princeton University Technical Advisory Council

E.A. DiMarzio, Member, Center for Excellence for Biomimetic Processing

Center for Molecular and Microstructure of Composites

D.L. Hunston, Member, Technical Advisory Board

Chemical Society of Washington

J.M. Antonucci

President, Program Chairman

Member, Long Range Planning Committee

B.B. Reed

Chairman, Science Fair Committee

Member, Long Range Planning Committee 
Member, Board of Manager

Comite International Permanent pour La Recherche Sur La Preservation des Materiaux en Milieu Marin (COIPM)

W.R. Blair, NIST Delegate

Composite Manufacturing

D.L. Hunston

Member, International Editorial Advisory Board

Defense Nuclear Agency

F.I. Mopsik, Member, Scientific Review Panel

Gordon Research Conference: Science of Adhesion

J.M. Antonucci

Member of the Council of the Gordon Research Conferences

L.C. Chow

Member of the Council of the Gordon Research Conferences

Gordon Research Conference: Polymer Physics

C.C. Han, Chairman 1994

Gordon Research Conference: Calcium Phosphates

L.C. Chow, Co-chairperson, 1992

Great Lakes Composites Consortium

D.L. Hunston, Member, Technical Advisory Board

Institute of Bioceramics, New York State College of Ceramics at Alfred University

L.A. George, Resource Board Member

Institute of Electrical and Electronic Engineers (IEEE)

Conference on Electrical Insulation and Dielectric Phenomena

A.S. DeReggi

Member, Executive Committee

Chairman, Digest Committee

Editor, Digest of Literature on Dielectrics

Institute for Interconnecting and Packaging Electronic Circuits (IPC)

M.A. Schen, NIST Representative, Technology Roadmap Committee

Interagency Oral Health Research Group

J.A. Tesk, Member

F.C. Eichmiller, Member 
International Association for Dental Research

J.M. Antonucci

Member, Executive Committee, Dental Materials Group

Archivist, Dental Materials Group

Member, Souder Award Committee

N.W. Rupp, Past President, Dental Materials Group

J.A. Tesk, Treasurer, Dental Materials Group

International Journal of Adhesion \& Adhesives

D.L. Hunston, Member, International Advisory Editorial Board

International Standards Organization

TC106, Dental Materials and Devices

F.C. Eichmiller, Pit and Fissure Sealants

J.A. Tesk

U.S. Expert, SC-2, WG-2; Base Metal Alloys

U.S. Expert, SC-2, WG-1 on Porcelain-Metal Systems and Dental Porcelain Member, TAG

J.R. Kelly

U.S. Expert, SC-2, WG-1; Porcelain-Metal Systems and Dental Porcelain

TC150, Cardiovascular Implants

J.A. Tesk, Advisor to TAG

Journal of Dental Research

J.R. Kelly, Editorial Review Board

J.A. Antonucci, Editorial Review Board

J.W. Stansbury, Editorial Review Board

L.C. Chow, Editorial Review Board

Journal of Operative Dentistry

F.C. Eichmiller, Member, Editorial Review Board

Journal of Polymer Science, Physics Edition

G.B. McKenna, Member, Editorial Review Board

C.C. Han, Member, Advisory Board

Journal of Rheology

G.B. McKenna, Member, Editorial Review Board

Guest Editor, Symposium on Rheology of Solids and Composites

MIT Club of DC

C.L. Schutte, Board of Directors 
Materials Research Society

C.L. Schutte, Session Organizer

Measurements for Polymer Processing Consortium

A.J. Bur, Member, Oversight Board

National Cancer Institute

J.A. Tesk, Consultant

National Institute Dental Research

J.M. Antonucci

AD HOC Reviewer of NIDR Grants

(SBIR) Grant Applications, Reviewer for SBIR Site Visits

L.C. Chow, AD HOC Reviewer of NIH Grants

J.W. Stansbury, Oral Biology and Medicine SBIR Grants Reviewer

National Institutes of Health

E.D. Eanes, Member, NIH Library Advisory Committee

National Science and Technology Council

M. A. Schen, Member, Sub-committee on Electronic Materials

NIST/Industry Polymer Blends Consortium

C.C. Han, Co-chairman

E.A. DiMarzio, Secretary

Polymer Composites

R.S. Parnas, Guest Editor

Polymers Division Editorial Committee

P.H. Verdier, Chairman

Semiconductor Equipment and Materials International

M.A. Schen, Task Leader, CTG Standard Test Method

Semiconductor Research Corporation

G. T. Davis, Member, Electronic Packaging Technical Advisory Board

M. A. Schen, Member, Electronic Packaging Technical Advisory Board

Society of Plastics Engineers

G.B. McKenna, Technical Program Committee Member for Plastics Analysis Division, 1994 ANTEC 
Society of Rheology

G.B. McKenna

Representative to American Institute of Physics Committee on Public Policy

U.S. Air Force

N.W. Rupp, Surgeon General's Consultant on Dental Materials, Emeritus

U.S. Naval Dental School

J.A. Tesk, Consultant

W.R. Rupp, Lecturer

F.C. Eichmiller, Lecturer

J.R. Kelly, Consultant

University of Lowell

F.W. Wang, Member, Plastics Advisory Group 
Industrial and Academic Interaction

\section{Industrial}

NIST is working with the Automotive Composites Consortium (ACC) formed by Ford, Chrysler, and General Motors to develop the technology which will make liquid composite molding the most attractive fabrication method for light-weight structural automotive parts. The technology permits the use of computer simulation rather than expensive, time consuming experiments to optimize tool design and processing conditions. NIST is using its process simulation models to predict mold filling for parts of interest to the ACC and to measure the preform permeabilities required by the analysis. The ACC provides details on the part design and feedback on the validity or the predictions from the simulation so the models can be refined and improved. A new project on the cross-member of a passenger van has begun.

General Electric and Ford Motor Company have an Advanced Technology Program that is attempting to commercialize a material system with the advantages of a thermoplastic but processes like a thermoset so that liquid composite molding can be used to make structural automotive parts. NIST's Polymers Division is supporting this effort by providing permeability measurements on the reinforcement materials to be used. These data are a key element in developing the optimized processing that is required to make the fabrication cost effective.

A cooperative program with Northrop-Grumman Corporation is extending NIST's process simulation capability for fabrication of structural composites parts by liquid molding to commercial aerospace applications. Grumman is testing and evaluating NIST's finite element codes through comparisons with flow visualization experiments and the fabrication of parts. NIST is using the results to refine and improve the models.

Boeing Aircraft is considering the use of RTM for fabrication of a variety of aircraft parts and is taking advantage of NIST's expertise in this area. The cooperation includes process simulation models and permeability measurements.

NIST, the Automotive Composites Consortium (Ford, General Motors, and Chrysler), Owens Corning, and Dow Chemical Company have initiated a joint program to study the fundamental aspects of durability for polymer composites in structural automotive applications. The goals are to improve test methods and predictive models for durability and to provide guidelines for the development of improved materials. NIST is studying environmental degradation in simple geometries like the single-fiber fragmentation test, and the ACC is investigating full composites to see how the fundamental data measured in the simple geometries translates to macroscopic composite properties. To assist the program, Owens Coring is providing glass fibers and expertise on surface treatments, while Dow Chemical Company is supplying resins and advice on sample preparation.

A collaborative program with Dow Chemical Company is investigating the effect of 
morphology on the fracture behavior of two-phase toughened thermosets that are important for adhesives and composites. Dow has some unique chemistry that permits the generation of materials with systematic variations in morphology. NIST is conducting fracture experiments and utilizing its expertise in toughening mechanisms to establish structure-property relationships that industry can use to formulate improved materials.

NIST is cooperating with the Textile Research Institute to develop new methods for measuring the permeability of preform materials that are deformed by placing in a mold and for automating the data acquisition in the fiber fragmentation test.

Under a Cooperative Research Agreement has been signed with Michigan Molecular Institute (MMI) NIST is using its small angle $x$-ray and neutron scattering facilities to study the size and shape of polyamidoamine dendrimers synthesized at MMI.

A NIST/industry consortium, "New Measurement Technology for Polymer Processing" develops real-time measurement technology based on optical and fluorescence methods to monitor important processing parameters. Member companies includes Dow Chemical, DuPont, 3M, Rohm \& Haas and Flow Vision.

Under a Research Associate Program with Enimont America a fluorescence method for in-line monitoring of a microwave cure process is being developed.

The NIST Small Angle X-ray and Pole Figure Facility continues to be a resource for the U.S. Industry. Projects on various aspects of effects of processing on the SAXS measured structure of polymers were performed with Mobil Chemical Company, Shell Oil Company. General Electric Corporation, W. R. Grace Company and Armstrong World Industries.

Collaborative studies of the yield behavior of glassy polymers were undertaken with Xerox.

A guest scientist from Xerox Corporation is engaged in a study of the evolution of the yield response of a copolymer glass after a quench from above to below the glass transition. The copolymer, polystyrene-poly(n-butyl methacrylate), is used in toner applications and the yield stress is related to the processing of the polymer into a fine powder for toner applications.

NIST and E. I. duPont de Nemours and Company have initiated a joint program to study the polymer/solid interface and to address the fundamental problem of packing density and interchain diffusion near a flat substrate. DuPont is supplying specialized model materials for the study which takes advantage of the new interface characterization technique based on neutron reflectivity developed by NIST. The results of the study will help identify processing conditions that optimize interface properties in composites, coatings, adhesives, and electronic packaging systems.

A Cooperative Research and Development Agreement has been established with the National Center for Manufacturing Science on the nature of the two phase morphology of 
interpenetrating polymer networks. One of the phases is produced from sol-gel chemistry based on organo-silicon compounds. The potential applications are to produce new materials for printed wiring boards. SANS studies have already started in collaboration with Sandia Labs, a consortium member of NCMS.

A Cooperative Research and Development Agreement was signed with the Dow Chemical Company to study fracture incubation in engineering thermoplastics using SAXS and position annihilation lifetime spectroscopy. Polymers Division staff and a foreign guest scientist collaborate with researchers from Dow to investigate incubation and growth of flows under creep loading conditions.

Collaborative research among scientists from NIST, Eastman Chemicals and the Forest Products Laboratory of the USDA investigates the morphology of cellulose triacetate and its aligomers by solid state NMR techniques.

Collaborative research among scientists from NIST and National Semiconductor Corporation is directed toward a fundamental understanding of moisture-induced cracking of microelectronic encapsulation subjected to solder reflow temperatures ("pop-corning").

A Cooperative Research and Development Agreement was developed among E. I. duPont de Nemours, Co., University of Delaware and NIST to study physical aging of Nylon ${ }^{\circledR}-66$, This resulted in a post-doctoral level researcher, I. Spinu, working in the Polymers Division.

A Cooperative Research Agreement with Armstrong World Industries studies the viscoelastic properties of plasticized PVC coatings. The work will use NIST's specialized characterization facilities to study the relationship between processing conditions and properties for model material systems provided by Armstrong. There is also a program to understand viscoelasticity, as it affects extrusion and foaming of rubber foam insulation.

Collaborative research among Goodyear, Sandia National Laboratory and NIST on the subject of elastomer blends involves SANS studies of the chemical microstructure effects on the miscibility of elastomer blends. NIST expertise on this subject are heavily relied upon in this technologically important subject.

A NIST/Industry polymer blends consortium was initiated to study effects on polymer blend properties of mixing/demixing under shear flow as well as methods of understanding and characterizing the interface. The first consortium meeting was held September 24, 1993. At that time five companies, Rohm and Haas, Raychem, 3M, Armstrong World Industries and Aristech had formally signed agreements to join the consortium. Goodyear Rubber and Tire Company and Sandia National Laboratories have also signed on since then. Negotiations with additional companies are still in progress.

Dr. Sigfried Bauer from the Heinrich Hertz Institute fur Nachrichtentechnik in Berlin participated in a study of polarization distribution in a poled polymer with non-linear optical 
properties. The study which used the NIST thermal pulse apparatus also led to a measurement of the thermal diffusivity of thin polyimide films adhered to a substrate.

Cooperative research with GE on the morphology and phase separation studies of the reactive polymerization system of cyclic polymers resulted in GE fellow, Dr. Roger Kambour spending 8 months in the Division working with the staff on this technologically important polymer system.

In cooperative research with the GM/GE ATP Program on Thermoplastic Engineering Design, Division staff are developing constitutive equations for nonlinear viscoelasticity of solid polymers. Experimental is also proceeding to validate viscoelastic models and to provide data for numerical simulations of post-mold dimensional stability.

A Dental and Medical Materials (DMMG) Group scientist took a lead role with American dental industry to incorporate changes to an ISO committee draft that had held clauses that would have had severe negative impacts on exporting some American dental casting alloys.

The DMMG Group is engaged in collaborative research with Dentsply/Caulk and the Metallurgy Division to develop a mercury free, direct-filling, metallic restorative.

Jeneric/Pentron signed an agreement for licensing of adhesive technology developed by American Dental Association Health Foundation (ADAHF) research associates in the dental group.

American Medical Electronics, Inc., licensed calcium phosphate cement technology from the ADAHF.

Colgate-Palmolive signed an option agreement with the ADAHF toward licensing a two-solution fluoride rinse developed by research associates from DMMG.

Health Tech Development, Inc. licensed an ADAHF patent on super-strong ternary alloys (Ru$\mathrm{Pd}-\mathrm{Zr}$ ). The company plans to develop the alloy for industrial, as well as dental, applications.

\section{Industrial/Academic}

A cooperative research program on the subject of polyolefin blends among NIST, Exxon and Princeton University has been carried out intensively in the past two years.

NIST is continuing to work with representatives from the microelectronics community who are interested in the quality and sophistication of measurements used for characterizing properties of microelectronic packaging materials. Z-axis coefficient of thermal expansion has been of primary interest during the past year. Currently, the group consists of representatives from Semiconductor Research Corporation, IBM (Endicott), DEC, Microelectronics and Computer Technology Corporation, Cornell University, University of Texas, University of 
Maryland, Purdue University, and Naval Surface Warfare Center (Crane, IN).

A workshop entitled "Materials Metrology and Data for Commercial Electrical and Optical Packaging and Interconnection Technologies" was held in Gaithersburg on May 5 and 6, 1994 and was cosponsored by NIST, The Institute for Interconnecting and Packaging Electronic Circuits, The Optoelectronics Industry Development Association, and The Semiconductor Research Corporation. Of the 109 in attendance, 45\% represented industrial companies, $18 \%$ came from universities and $37 \%$ were from government agencies. The purpose of the workshop was to augment industry roadmapping efforts by identifying priorities in materials data and measurement sciences, bridging the gap between suppliers, manufacturers and assemblers, as well as catalyze collaborations among the various segments of the electronics industry. Results of the workshop are summarized in NISTIR 5520.

\section{Academic}

A grant to Drexel University supports development of optical and ultrasonic techniques to measure resin flow front velocity, mold filling time, resin solidification, resin temperature, part shrinkage, and resin modulus.

Professor K. F. Lin, National Taiwan University, participated in the development of a fluorescence technique, based on frequency shifts, to measure curing of thermosetting resins. The approach has the advantage that no internal standard is needed.

A NATO sponsored collaborative effort with the Instifuto de Ciencia y Tecnologia de Polimeros, Madrid, Spain investigates smectic liquid crystalline polymers.

A collaborative project with the University of Maryland Baltimore funded by the EPA addresses the stability of well-characterized lead solutions in polycarbonate bottles. The solutions are used in toxicology studies at the University of Maryland.

Staff at the University of Massachusetts Lowell collaborates on the development of an in-line method for monitoring the uniformity of mixing in blends of recycled plastics.

Professor H. Marand of Virginia Polytechnic Institute and State University joins in the study of the fine texture of crystallizable polymer blends.

The measurement of domain size and stoichiometry in blends of cellulose and hydrogen-bonded polymers by solid state nuclear magnetic resonance spectroscopy is conducted jointly with McGill University.

A collaboration with Tel Aviv University investigates failure mechanisms in adhesive joints by analysis of experimental results by elasto-plastic finite element methods.

A collaborative program has been initiated with the University of Delaware to investigate the 
polymer/solid interface in composites. Pearl Chin, a PhD student of Prof. Roy L. McCullough, uses the NIST-developed neutron reflectivity technique to determine the polymer microstructure near the interface. The results will be correlated with other experiments conducted at both NIST and Delaware to assess interface properties. The goal is to provide the understanding needed to optimize the interface structure for the best composite performance.

Experimental studies of the phase diagrams and dynamics of polymer blends are conducted with collaborators from Kyoto University, Massachusetts Institute of Technology, University of Michigan, University of Maryland, Cornell University, Princeton University, Tokyo Science University, and East China University of Chemical Technology.

Theoretical studies of phase dynamics in polymer blends is conducted with Professor M. Muthukumar of the University of Massachusetts.

A joint project with Professor N. Tsutsumi of the Kyoto Institute of Technology investigates polymer blends as host materials for non-linear optically active guest molecules.

A joint program is underway between NIST and Prof. John Hoffman at Johns Hopkins University to study on-line process monitoring in liquid composite molding. As part of this effort a PhD student, Dara Woerdeman, is working at NIST to develop an evanescent-wave, optical-fiber, fluorescence measurement. This work will permit detailed measurements of the local resin behavior deep within a composite part during processing.

A joint project with Prof. A. S. Wineman of the University of Michigan investigates the mechanical response of compressible viscoelastic materials.

A joint program has been initiated with Prof. Suresh Advani at the University of Delaware to investigate liquid molding. The interaction involves the development of micro-flow models in an effort to predict permeability and an experimental study to visualize and model 3-dimensional flow effects in multi-layer preforms. A Postdoctoral Fellow from the University, Sridhar Ranganathan, is working at NIST on the modelling effort.

A joint program was initiated with Prof. Raghavan and $\mathrm{H}$. Huynh of Howard University to study the use of rubber particles from recycled tires as additives to concrete for roads and other applications. This offers the hope of helping to solve the tire recycling problem as well as the development of improved concretes.

Professor Susumer Ikeda from Yamagatu University in Yonezawa, Japan was a guest scientist during his sabbatical leave. He worked with us in developing a precision capacitor for the measurement of $\mathrm{z}$-axis thermal expansion of thin polymer films.

The Dental and Medical Materials Group participated in collaborative research efforts with scientists from the following academic institutions: The University Louis Pasteur (France), Nihon University (Japan), Fukuoka Dental College (Japan), Kyushu University (Japan), the 
Rudjer Boskovic Institute (Yugoslavia), the University of Maryland, the University of Florida, the Oregon Health Sciences University, Taiwan University, Howard University, the U.S. Naval Dental School (Bethesda), the University of Strasbourg (France), McGill University (Canada) and the West China University of Medical Sciences. Research projects including studies of stress in porcelain-metal and other dental bonded systems, new coupling agents for dental composites, postcure properties of dental composites, polymeric calcium phosphate cements, synthesis and characterization of novel fluoro and siloxane monomers and oligomers, implants biomineralization, and calcium phosphate chemistry.

A joint project with Prof. A. Lee of Michigan State University is investigating the physical aging behavior of rubber toughened epoxy resins in creep and stress relaxation. 


\section{Associated Activities}

\section{Invited Talks}

Division personnel presented a total of 96 invited talks on research activities at the following types of organizations and symposia: industrial laboratories, 11; international symposia, 8; universities, 33; topical symposia, 6; national and government laboratories, 8; professional society meetings, 24; and Gordon Conferences, 6.

\section{Patents}

Hydroxyfluoroalkyl-substituted Styrenes and Polymer Compositions Containing Same. C.C. Han, M. He, Y. Liu, Y. Feng and M. Jiang, PTO 5,352,742.

Method for Detecting Thermodynamic Phase Transitions during Polymer Injection Molding. A.J. Bur, F.W. Wang, C.L. Thomas and J.L. Rose, PTO 5,384,079.

Method and Apparatus for Monitoring Resin Crystallization and Shrinkage during Polymer Molding. A.J. Bur and C.L. Thomas, (Pending).

Improved Monomers for Double Ring Opening Polymerization with Expansion. J.W. Stansbury, PTO 5,362,889.

Novel Multifunctional Acrylates and the Synthesis Thereof. J.M. Antonucci, J.W. Stansbury and G.W. Chen, PTO 5,380,901.

A Simple Effective Method for Bonding to Mineralized Dental Tissue Using Nphenylininodiacetic Acid. J.M. Antonucci and P. Bennett, (Pending).

Use of Pre-Ceramic Polymers in Fabrication of Interpenetrating Phase Composites. J.M. Antonucci and J.R. Kelly, (Pending).

Visible Light Photoinitiators for Free Radical, Cationic and Dual Polymerizations of Dental Resins. J.M. Antonucci, J.W. Stansbury and C. Gingreau, (Pending).

Improved Dental Compositions via Improved Interfaces. J.M. Antonucci and J.W. Stansbury, (Pending).

Process for Forming Alloys In Situ in Absence of Liquid-Phase Sintering. D.S. Lashmore, J.A. Tesk, M.P. Dariel, E. Escalante, PTO 5,318,746.

Electroforming of Metallic Glasses for Dental Applications. M. Ratzker, D.S. Lashmore and J.A. Tesk, PTO 5,316,650. 

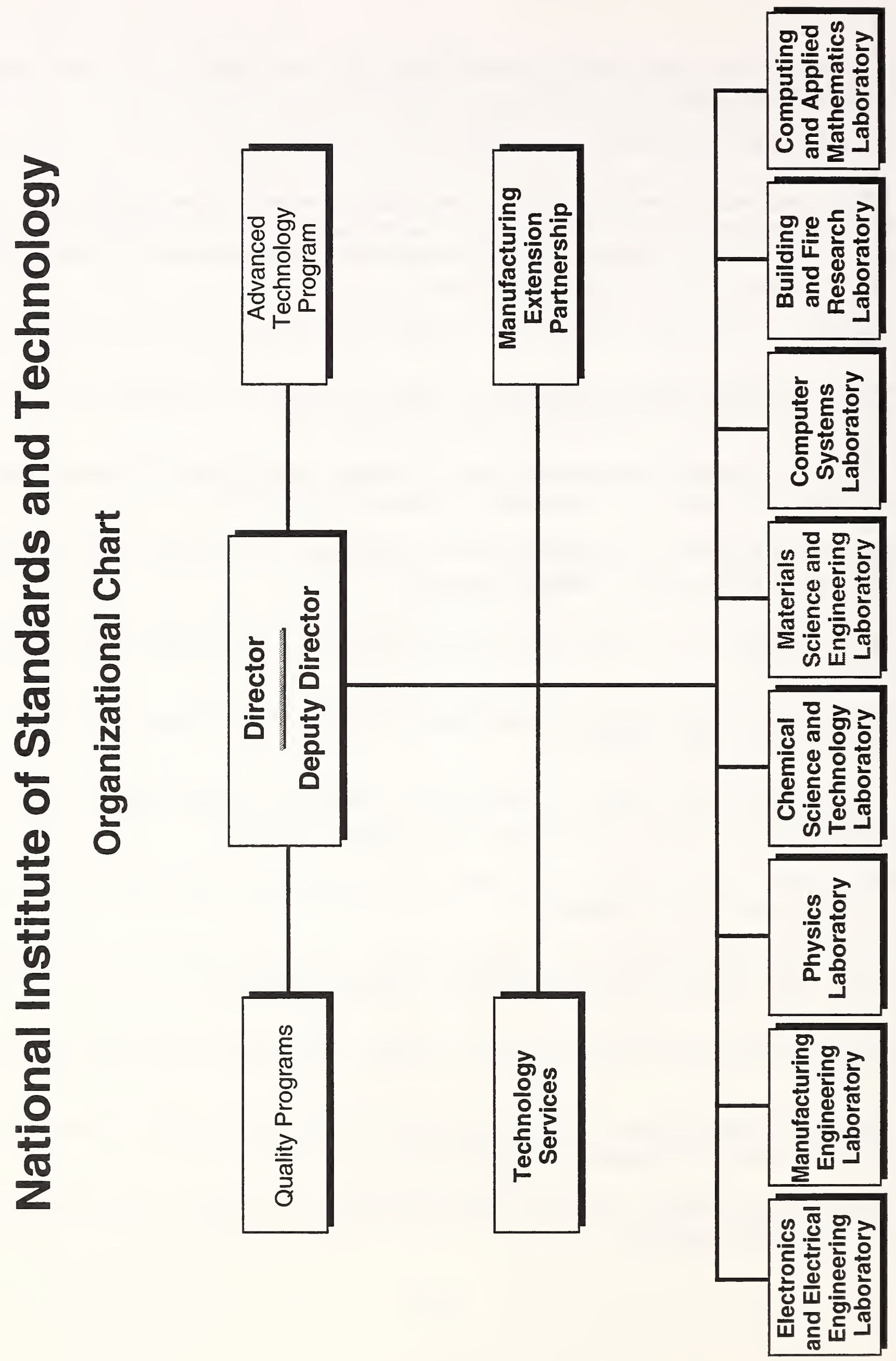


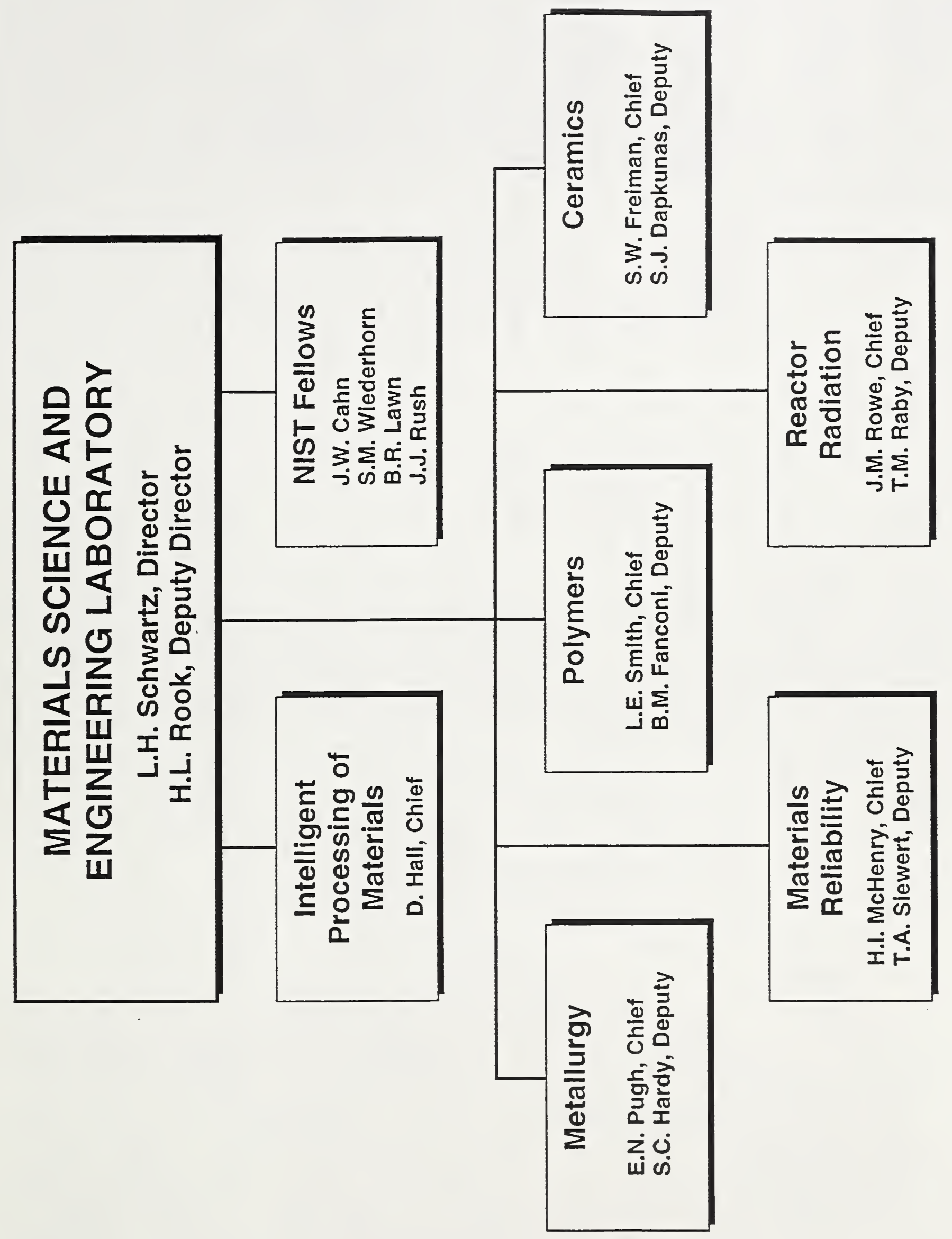




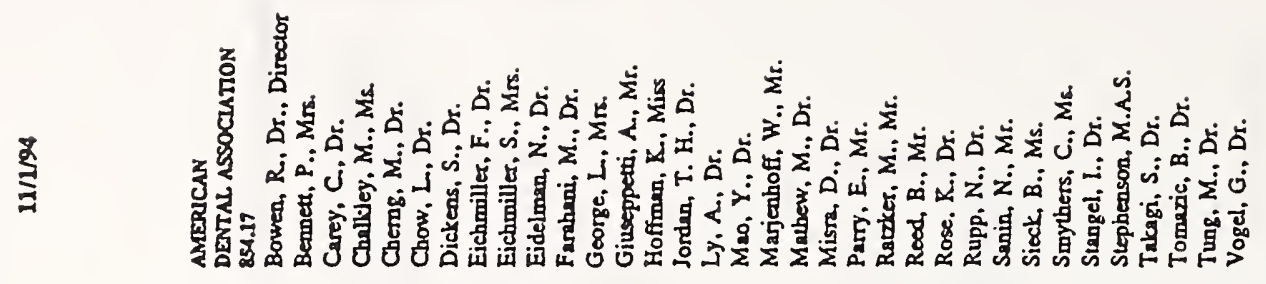

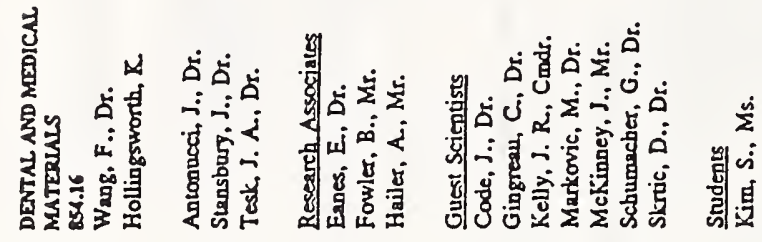

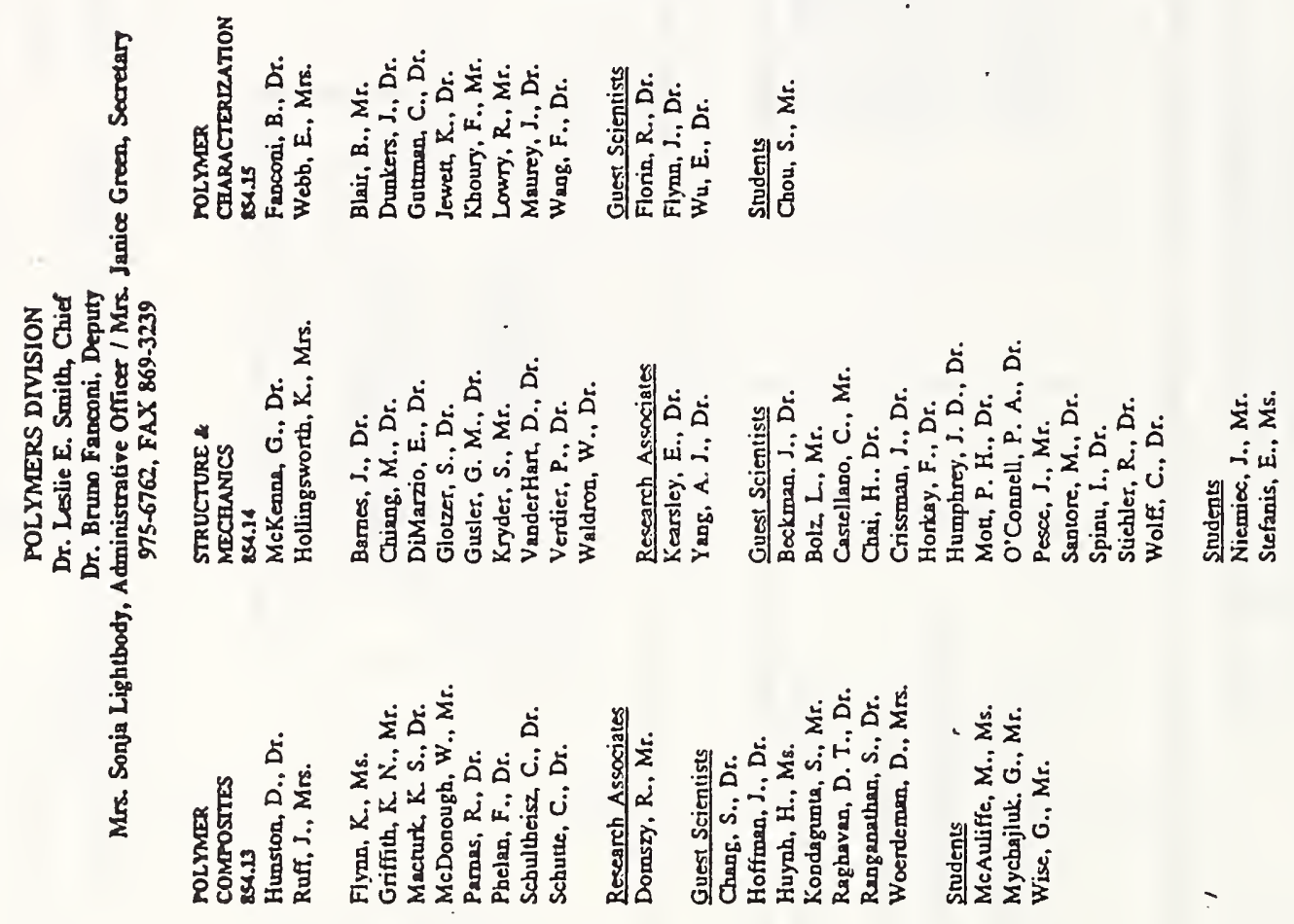

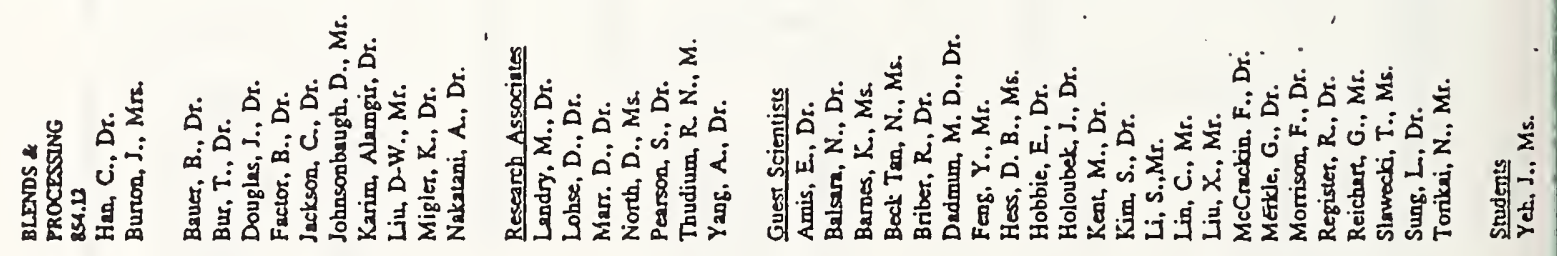

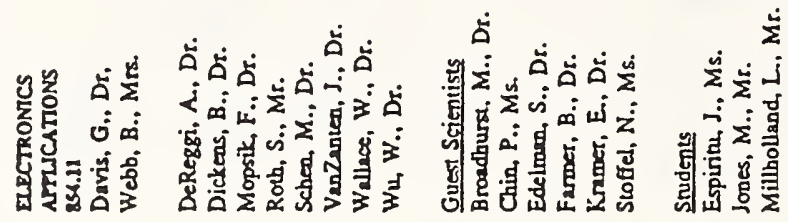


Probabilistic Calculation of the Hydrodynamic Radius of a Polymer Chain. The figure shows a representative polymer (spheres) and a random walk trajectory (filamentary path) which is used to probe the polymer structure. The random walks are launched at random points from an enclosing sphere and the friction coefficient is calculated from the fraction of random walk trajectories which hit the polymer before returning to the sphere from which they initiated. The probabilistic algorithm applies to essentially arbitrarily-shaped particles and other transport properties of polymer solutions and blends can be calculated using similar methods. The figure was prepared by Robert Lipman of the Scientific Visualization Group/CAML. 
